UTMS 94-35

hep-th/9405096

\title{
INTEGRABLE HIERARCHIES AND DISPERSIONLESS LIMIT
}

\author{
KANEHISA TAKASAKI \\ Department of Fundamental Sciences, \\ Faculty of Integrated Human Studies, Kyoto University, \\ Yoshida-Nihonmatsu-cho, Sakyo-ku, Kyoto 606, Japan \\ E-mail: takasaki@jpnyitp (Bitnet) \\ TAKASHI TAKEBE \\ Department of Mathematical Sciences, University of Tokyo, \\ Hongo, Bunkyo-ku, Tokyo 113, Japan, \\ E-mail: takebe@math.s.u-tokyo.ac.jp
}

\begin{abstract}
Analogues of the KP and the Toda lattice hierarchy called dispersionless KP and Toda hierarchy are studied. Dressing operations in the dispersionless hierarchies are introduced as a canonical transformation, quantization of which is dressing operators of the ordinbry KP and Toda hierarchy. An alternative construction of general solutions of the ordinary KP and Toda hierarchy is given as twistor construction which is quatization of the similar construction of solutions of dispersionless hierarchies. These results as well as those obtained in previous papers are presented with proofs and necessary technical details.
\end{abstract}

\section{INTRODUCTION}

This article aims to present a comprehensive overview of our work [1-4] on a kind of non-linear integrable systems, so-called "dispersionless hierarchies" which arise as quasiclassical limit of the KP hierarchy and the Toda lattice hierarchy. Our standpoint here is that the ordinary $\mathrm{KP} /$ Toda hierarchies describe a quantized canonical transformation of a canonical transformation determined by the dispersionless hierarchies. 
This kind of integrable systems was introduced by Lebedev, Manin and Zakharov [5]. Many special solutions were obtained by Kodama and Gibbons [6]. We were led to this subject, however, by recent developments in low-dimensional quantum field theories. One of our motivations is the work of Krichever [7] which studies the "dispersionless Lax equations" (= the dispersionless KP hierarchy) and introduced the analogue of the tau function to integrate the consistency conditions (the WDVV equations in the terminology of [8]) for the free energy of the topological minimal models (Landau-Ginzburg description of the A-type minimal models). Another motivation is the dispersionless Toda equation that was then studied in the context of Einstein equations ([9]), integrable systems connected with "continuous Lie algebras" ([10]), extended conformal symmetries $\left(w_{1+\infty}\right.$ algebras) $([11])$, and twistor theory $([12])$.

Inspired by these observations, we made attempts to apply the approach pioneered by Sato $([13],[14],[15])$ to these dispersionless hierarchies. Our earlier works $[1,3]$ are based on the twistor theoretical viewpoint developed in $[16,17]$. We started from a classical canonical conjugate pair $(\mathcal{L}, \mathcal{M}),\{\mathcal{L}, \mathcal{M}\}=1$, where $\mathcal{L}$ is an analogue of the usual Lax operator $L$ and $\mathcal{M}$ is an analogue of the Orlov operator $M$ conjugate to $L:[L, M]=1$ which was first introduced by Orlov to study the symmetries of the KP hierarchy $([18])$. (For the case of the (dispersionless) Toda hierarchy these pairs $(\mathcal{L}, \mathcal{M})$, $(L, M)$ are doubled.) Regarding $\mathcal{L}, \mathcal{M}$ as Darboux coordinates, we introduced the $S$ function and the tau function found by Krichever, gave twistor theoretical construction of general solutions (a kind of Riemann-Hilbert decomposition) and described $w_{1+\infty}$ symmetries (SDiff(2) symmetries in the terminology of $[1,3]$ ).

It turned out in $[2,4]$ that these ingredients of dispersionless hierarchies come from the ordinary KP/Toda hierarchy as quasi-classical limit in the sense of the WKB analysis. For example, the $S$ function is the phase function of the solutions of linear problems (the Baker-Akhiezer functions) and the Lax equations arise as the Hamilton-Jacobi (eikonal) equations. 
More "traditional" approach to integrable hierarchies is different from the above method. Usually the system is converted into the differential equations for the dressing operators, which determine the properties of the Baker-Akhiezer functions, the tau functions and so on.

In the present paper, we show that this approach with the dressing operation is also applicable to the dispersionless hierarchies: starting from a single series $\mathcal{L}$ instead of using the pair $(\mathcal{L}, \mathcal{M})$, we introduce a dressing operation in Lie-algebraic language. The Orlov function $\mathcal{M}$ is now defined by means of this dressing operation. The existence of the twistor data for a solution is proved and the infinitesimal $\left(w_{1+\infty}\right)$ symmetries of $\mathcal{L}$, $\mathcal{M}$ are shown to be a consequence of the symmetry of the dressing function.

In order to make the meaning of quasi-classical limit more transparent, we import such notions as "orders" and "principal symbols" of micro-differential operators from the theory of linear partial differential equations, regarding the Planck constant $\hbar$ as $\partial_{t_{0}}^{-1}$, where $t_{0}$ is an extra time variable. In this context, dressing operations of the ordinary $\mathrm{KP} /$ Toda hierarchies are nothing but quantization of dressing operations of the dispersionless hierarchies.

We present our new results along with reviews on previous ones [1-4] and give proofs absent from them so as to make the article self-contained. This paper consists of two parts. The first part is devoted to the dispersionless KP hierarchy and the second part to the dispersionless Toda hierarchy. Both parts are organized in a parallel way: In section 1 , we define the system in terms of the Lax equations for $\mathcal{L}$ (and $\overline{\mathcal{L}}$ ). We introduce the dressing function in section 2, with the help of which we define the Orlov function $\mathcal{M}$ (and $\overline{\mathcal{M}}$ ) in section 3 . Section 4 is a review on the $S$ function and the tau function. In section 5 solutions of dispersionless hierarchies are constructed by the twistor theoretical method or, in other words, by the Riemann-Hilbert decomposition. This construction provides all the solutions as we shall prove by means of the dressing operation. Deformations of the input of this construction, i.e., the twistor data, 
we define $w_{1+\infty}$ symmetries of solutions and the tau functions in section 6 . Section 7 contains six subsections which correspond to section 1-6 above. In these subsections we describe the counterpart of section 1-6 in the theory of the ordinary $\mathrm{KP} /$ Toda hierarchies for which quasi-classical limit is well-defined and reproduces what we considered in the previous sections. In fact, the dressing operators have a quite specific form which makes it possible to take the quasi-classical limits of $\mathcal{L}, \mathcal{M}$ etc., and thus we may regard the dressing operation of the ordinary $\mathrm{KP} /$ Toda hierarchies as the quantized contact transformation [19]. We also give an alternative method of constructing all solutions of these ordinary hierarchies from the twistor theoretical viewpoint. This is a kind of Riemann-Hilbert decomposition, but not that one considered in [20], [21]. To illustrate how this theory works, we make a brief review on special solutions which appear in the study of topological strings in section 8 of the part 1. We refer to [22] for an application of the dispersionless Toda hierarchy to the theory of the two dimensional string theory. As is expected from the theory of the ordinary Toda lattice hierarchy [21], solutions of the dispersionless Toda hierarchy give solutions of the dispersionless KP hierarchy if one fixes certain variables. We prove this fact directly in section 8 of part 2 .

Several facts on formal properties of differential algebras are gathered in appendix A which are necessary especially for study of dressing operations. In appendix B we give quasi-classical limits of certain Hirota equations of the KP hierarchy which conjecturally characterize the tau function of the dispersionless KP hierarchy.

The authors express gratitude to M. Noumi, A. Yu. Orlov, T. Shiota for their useful comments. This work is partly supported by the Grant-in-Aid for Scientific Research, the Ministry of Education, Science and Culture, Japan.

After completing this work, Yuji Kodama informed us that the dressing transformation in $\S 1.2$ of the present paper is directly connected with the canonical transformation introduced by Kodama and J. Gibbons [43]. They use this transformation to transform 
the system into a separable form with canonical variables of action-angle type which correspond to $\mathcal{L}$ and $\mathcal{M}$ in our notation. We thank Yuji Kodama for this comment.

\section{DiSPERSIONLESS KP HIERARCHY}

\subsection{Lax formalism.}

Here we briefly review the definition of the dispersionless KP hierarchy, mostly following $[1,3]$. The dispersionless KP hierarchy has a Lax representation with respect to a series of independent ("time") variables $t=\left(t_{1}, t_{2}, \ldots\right)$

$$
\frac{\partial \mathcal{L}}{\partial t_{n}}=\left\{\mathcal{B}_{n}, \mathcal{L}\right\}, \quad \mathcal{B}_{n} \underset{\text { def }}{=}\left(\mathcal{L}^{n}\right)_{\geq 0}, \quad n=1,2, \ldots
$$

where $\mathcal{L}$ is a Laurent series in an indeterminant $k$ of the form

$$
\mathcal{L}=k+\sum_{n=1}^{\infty} u_{n+1}(t) k^{-n}
$$

"( $)_{\geq 0}$ " means the psojection onto a polynomial in $k$ dropping negative powers, and " $\{\quad\}$ " the Poisson bracket in 2D "phase space" $(k, x)$,

$$
\{A(k, x), B(k, x)\}=\frac{\partial A(k, x)}{\partial k} \frac{\partial B(k, x)}{\partial x}-\frac{\partial A(k, x)}{\partial x} \frac{\partial B(k, x)}{\partial k}
$$

Thus the dispersionless KP hierarchy (1.1.1) is a collection of non-linear differential equations for $u_{n}(t)$ with respect to $t$. In particular, (1.1.1) for $n=1$ says that $\mathcal{L}$ depends on $t_{1}$ and $x$ through the combination $t_{1}+x$, like the ordinary KP hierarchy. This system is apparently the "total symbol" of the KP hierarchy obtained by replacing

microdifferential operators (in $x$ ) and their commutators by Laurent series (in $k$ ) and Poisson brackets. In fact we shall see in $\S 1.7$ that this is a kind of the "principal symbol" of the KP hierarchy.

By imitating the usual argument for the ordinary KP hierarchy (cf., e.g., [15]), one can easily prove the following fact 
Proposition 1.1.1. The Lax equations for $\mathcal{L}$ are equivalent to the "zero-curvature equations"

$$
\frac{\partial \mathcal{B}_{m}}{\partial t_{n}}-\frac{\partial \mathcal{B}_{n}}{\partial t_{m}}+\left\{\mathcal{B}_{m}, \mathcal{B}_{n}\right\}=0
$$

and to its "dual" form

$$
\frac{\partial \mathcal{B}_{m}^{-}}{\partial t_{n}}-\frac{\partial \mathcal{B}_{n}^{-}}{\partial t_{m}}-\left\{\mathcal{B}_{m}^{-}, \mathcal{B}_{n}^{-}\right\}=0
$$

where

$$
\mathcal{B}_{n}^{-} \underset{\text { def }}{=} \mathcal{L}^{n}-\mathcal{B}_{n}=\left(\mathcal{L}^{n}\right) \leq-1
$$

and $(\quad)_{\leq-1}$ stands for the negative power part of Laurent series of $k$.

In [1] we formulated the hierarchy in terms of two Lax series $\mathcal{L}$ and $\mathcal{M}$, and assumed the existence of $\mathcal{M}$ from the beginning. In fact the Lax equation (1.1.1) of $\mathcal{L}$ closes in itself, and one can start from (1.1.1) without postulating the existence of $\mathcal{M}$. We shall show below that even in that setting one can construct $\mathcal{M}$. To this end we need a counterpart of the dressing operator.

\subsection{Dressing operators.}

As we remarked in [1], $\S 3$, we have no direct counterpart of the dressing operator $W$ of the KP hierarchy, i.e., a monic 0-th order micro-differential operator $W$ that satisfies the equations

$$
L_{\mathrm{KP}}=W \partial W^{-1}, \quad \frac{\partial W}{\partial t_{n}} W^{-1}=-\left(W \partial^{n} W^{-1}\right)_{\leq-1}, \quad n=1,2, \ldots
$$

where ()$_{\leq-1}$ means the projection of micro-differential operators onto the negative order part. Note, however, that the operator $W$ arises in (1.2.1) as an adjoint action in the Lie algebra $\mathcal{E}(-1)$ of micro-differential operators of negative order. Writing $W$ as $W=\exp (X), X \in \mathcal{E}(-1)$, one can indeed consider (1.2.1) as a collection of evolution equations for the negative order micro-differential operator $X$. The dressing operation $P \mapsto W P W^{-1}$ is nothing but the adjoint action $P \mapsto \exp (\operatorname{ad}(X)) P$. 
This observation suggests to define a dressing operators of the dispersionless KP hierarchy as a group element $\exp (\operatorname{ad} \varphi)$ of the Lie algebra of Poisson brackets. One can indeed find such a dressing operator in the following manner.

Proposition 1.2.1. i) Let $\mathcal{L}$ be a solution of the dispersionless KP hierarchy (1.1.1). Then there exists a Laurent series $\varphi$ (dressing fucntion) that satisfies the equations

$$
\mathcal{L}=e^{\operatorname{ad} \varphi}(k), \quad \nabla_{t_{n}, \varphi} \varphi=-\left(e^{\operatorname{ad} \varphi}\left(k^{n}\right)\right)_{\leq-1}, \quad n=1,2, \ldots
$$

of the form $\varphi(t)=\sum_{n=1}^{\infty} \varphi_{n}(t) k^{-n}$, where

$$
\operatorname{ad} \varphi(\psi)=\{\varphi, \psi\}, \quad \nabla_{t_{n}, \psi} \varphi=\sum_{m=0}^{\infty} \frac{1}{(m+1) !}(\operatorname{ad} \psi)^{m}\left(\frac{\partial \varphi}{\partial t_{n}}\right) .
$$

Such Laurent series $\varphi$ is not unique, but non-uniqueness is limited to replacing $\varphi \mapsto$ $H(\varphi, \psi)$, with a constant Laurent series $\psi=\sum_{n=1}^{\infty} \psi_{n} k^{-n}\left(\psi_{n}\right.$ : constant), where $H(X, Y)$ is the Hausdorff series (cf. Appendix A) defined by

$$
\exp (\operatorname{ad} H(\varphi, \psi))=\exp (\operatorname{ad} \varphi) \exp (\operatorname{ad} \psi)
$$

ii) Conversely, if $\varphi(t)=\sum_{n=1}^{\infty} \varphi_{n}(t) k^{-n}$ satisfies the second equations of (1.2.2), then $\mathcal{L}$ defined in (1.2.2) is a solution of the dispersionless KP hierarchy (1.1.1).

Proof. i) It is easy to see that there exists a formal power series $\varphi^{0}(t)=\sum_{n=1}^{\infty} \varphi_{n}^{0} k^{-n}$ such that

$$
\mathcal{L}=e^{\operatorname{ad} \varphi^{0}}(k)
$$

We now modify $\varphi^{0}(t)$ to reach a desired function $\varphi(t)$ satisfying (1.2.2).

Using Lemma A.1 in Appendix A, we can rewrite the Lax equations (1.1.1) into the form

$$
\left\{e^{-\operatorname{ad} \varphi^{0}} \nabla_{t_{n}, \varphi^{0}} \varphi^{0}, k\right\}=\left\{e^{-\operatorname{ad} \varphi^{0}} \mathcal{B}_{n}, k\right\}
$$

Since $\{k, \cdot\}=\partial / \partial x$, uhis means that $\mathcal{B}_{n}^{\varphi^{0}} \underset{\text { def }}{=} e^{-\operatorname{ad} \varphi^{0}}\left(\mathcal{B}_{n}-\nabla_{t_{n}, \varphi^{0}} \varphi^{0}\right)$ does not depend on $x$. On the other hand, the zero-curvature equations (1.1.4) imply

$$
\frac{\partial \mathcal{B}_{m}^{\varphi^{0}}}{\partial t_{n}}-\frac{\partial \mathcal{B}_{n}^{\varphi^{0}}}{\partial t_{m}}+\left\{\mathcal{B}_{m}^{\varphi^{0}}, \mathcal{B}_{n}^{\varphi^{0}}\right\}=0
$$


due to Lemma A.3. Since $\partial \mathcal{B}_{n}^{\varphi^{0}} / \partial x=0$ for any $n$, we have $\left\{\mathcal{B}_{n}^{\varphi^{0}}, \mathcal{B}_{m}^{\varphi^{0}}\right\}=0$. Therefore (1.2.4) is nothing but the compatibility coditions $\partial_{n} \mathcal{B}_{m}^{\varphi^{0}}=\partial_{m} \mathcal{B}_{m}^{\varphi^{0}}$ of the system

$$
\frac{\partial \varphi^{\prime}(t)}{\partial t_{n}}=k^{n}-\mathcal{B}_{n}^{\varphi^{0}}
$$

for an unknown function $\varphi^{\prime}(t)=\sum_{n=1}^{\infty} \varphi_{n}^{\prime}(t) k^{-n}$. (Note that by definition $\mathcal{B}_{n}^{\varphi^{0}}$ has uhe

form $k^{n}+\sum_{m=1}^{\infty} b_{n, m}^{0} k^{-m}$.) Since $\mathcal{B}_{n}^{\varphi^{0}}$ does not depend on $x$, we can choose $\varphi^{\prime}(t)$ to be independent of $x$ (though possibly depending on $t_{1}$ ).

By virtue of the Hausdorff-Campbell formula there exists a series $\varphi(t)$ satisfying $\exp (\operatorname{ad} \varphi)=\exp \left(\operatorname{ad} \varphi^{\prime}\right) \exp \left(\operatorname{ad} \varphi^{0}\right)$. Recalling that $\partial \varphi^{\prime} / \partial x=0$, we have

$$
\begin{aligned}
e^{-\operatorname{ad} \varphi}\left(\mathcal{B}_{n}-\nabla_{t_{n}, \varphi} \varphi\right) & =e^{\operatorname{ad} \varphi^{\prime}} \mathcal{B}_{n}^{\varphi^{0}}+\nabla_{t_{n}, \varphi^{\prime}} \varphi^{\prime} \\
& =k^{n}
\end{aligned}
$$

because of (1.2.5) and Lemma A.2. Therefore

$$
\mathcal{B}_{n}=e^{\operatorname{ad} \varphi} k^{n}+\nabla_{t_{n}, \varphi} \varphi, \quad n=1,2, \ldots
$$

which is equivalent to the second equations of (1.2.2). The only ambiguity of the above construction of $\varphi$ is that of $\varphi^{\prime}$, which can be absorbed into the transformation $\varphi \mapsto H(\varphi, \psi)$ with a suitable constant series $\psi$.

ii) is a direct consequence of Lemma A.1.

\subsection{Orlov function and Darboux coordinates.}

Having obtained the dressing operator $\exp (\operatorname{ad} \varphi)$, we can reproduce the dispersionless analogue $\mathcal{M}$ of the Orlov operator $M$ of the KP iierarchy.

The Orlov function $\mathcal{M}$ is by definition a formal Laurent series

$$
\begin{aligned}
\mathcal{M} & =e^{\operatorname{ad} \varphi}\left(x+\sum_{n=1}^{\infty} n t_{n} k^{n-1}\right) \\
& =e^{\operatorname{ad} \varphi} e^{\operatorname{ad} t(k)}(x),
\end{aligned}
$$

where $t(k)=\sum_{n=1}^{\infty} t_{n} k^{n}$. It is convenient to expand $\mathcal{M}$ into a Laurent series of $\mathcal{L}$ as:

$$
\mathcal{M}=\sum_{n=1}^{\infty} n t_{n} \mathcal{L}^{n-1}+x+\sum_{i=1}^{\infty} v_{i} \mathcal{L}^{-i-1}
$$


Proposition 1.3.1. i) The series $\mathcal{M}$ satisfies the Lax equation

$$
\frac{\partial \mathcal{M}}{\partial t_{n}}=\left\{\mathcal{B}_{n}, \mathcal{M}\right\}, \quad n=1,2, \ldots
$$

and the canonical Poisson relation $\{\mathcal{L}, \mathcal{M}\}=1$.

ii) Conversely, if a series $\mathcal{M}$ of the form (1.3.2) satisfies (1.3.3) and the canonical Poisson relation, then there exists a unique dressing function $\varphi$ which is connected with $\mathcal{M}$ through (1.3.1). Explicitly, $\varphi(t)=\sum_{n=1}^{\infty} \varphi_{n}(t) k^{-n}$ is of the form

$$
\varphi_{n}(t)=-\frac{v_{n}}{n}+\left(\text { a differential polynomial of } v_{1}, \ldots, v_{n-1}, u_{2}, \ldots, u_{n-1}\right) \text {. }
$$

Proof. i) The canonical conjugation relation is obvious from the definition. The Lax equation (1.3.3) can be derived as:

$$
\begin{aligned}
\partial_{t_{n}} \mathcal{M} & =\left\{\nabla_{t_{n}, \varphi} \varphi, \mathcal{M}\right\}+e^{\operatorname{ad} \varphi}\left(n k^{n-1}\right) \\
& =\left\{\mathcal{B}_{n}-e^{\operatorname{ad} \varphi} k^{n}, \mathcal{M}\right\}+e^{\operatorname{ad} \varphi}\left(n k^{n-1}\right) \\
& =e^{\operatorname{ad} \varphi}\left(\left\{e^{-\operatorname{ad} \varphi} \mathcal{B}_{n}-k^{n}, \sum_{n=1}^{\infty} n k^{n-1}+x\right\}+n k^{n-1}\right) \\
& =\left\{\mathcal{B}_{n}, \mathcal{M}\right\}
\end{aligned}
$$

because of Lemma A.1.

ii) Let $\varphi^{0}$ be a dressing function determined by Proposition 1.2.1. From the canonical Poisson relation we get

$$
1=e^{-\operatorname{ad} \varphi^{0}}\{\mathcal{L}, \mathcal{M}\}=\left\{k, e^{-\operatorname{ad} \varphi^{0}} \mathcal{M}\right\}
$$

Hence $\exp \left(-\operatorname{ad} \varphi^{0}\right) \mathcal{M}$ has the form

$$
e^{-\operatorname{ad} \varphi^{0}} \mathcal{M}=\sum_{n=1}^{\infty} n t_{n} k^{n-1}+x+\sum_{i=1}^{\infty} v_{i}^{0}(t) k^{-i-1}
$$

where the coefficients $v_{i}^{0}(t)$ do not depend on $x$. On the other hand, from Lemma A.1, the Lax equation (1.3.3) and (1.2.6), we have 


$$
\begin{aligned}
\frac{\partial}{\partial t_{n}}\left(e^{-\operatorname{ad} \varphi^{0}} \mathcal{M}\right) & =e^{-a d \varphi^{0}}\left(\frac{\partial \mathcal{M}}{\partial t_{n}}-\left\{\nabla_{t_{n}, \varphi^{0}} \varphi^{0}, \mathcal{M}\right\}\right) \\
& =\left\{k^{n}, e^{-\operatorname{ad} \varphi^{0}} \mathcal{M}\right\}
\end{aligned}
$$

Together with (1.3.6), this means $\partial v_{i}^{0}(t) / \partial t_{n}=0$ for all $n$ and $i$. Thus

$$
e^{\operatorname{ad} \sum_{i=1}^{\infty} v_{i}^{0} k^{-i} / i} e^{-\operatorname{ad} \varphi^{0}} \mathcal{M}=\sum_{n=1}^{\infty} n t_{n} k^{n-1}+x,
$$

and $\varphi=H\left(\varphi^{0}, \sum_{i=1}^{\infty} v_{i}^{0} k^{-i} / i\right)$ gives a desired dressing function. One can easily show (1.3.4) by ioduction, noting that

$$
x+\sum_{i=1}^{\infty} v_{i}(t) \mathcal{L}^{-i-1}=e^{\operatorname{ad} \varphi(t)} x .
$$

Uniqueness of $\varphi$ is obvious from the construction.

One of the fundamental properties of $\mathcal{M}$ is that the $(\mathcal{L}, \mathcal{M})$-pair gives a Darboux coordinate system of the 2 -form

$$
\omega \underset{\text { def }}{=} \sum_{n=1}^{\infty} d \mathcal{B}_{n} \wedge d t_{n}=d k \wedge d x+\sum_{n=2}^{\infty} d \mathcal{B}_{n} \wedge d t_{n},
$$

where " $d$ " now stands for total differentiation in both $t$ and $k$. This 2 -form is actually a degenerate symplectic form because: (i) from the definition, $\omega$ is a closed form,

$$
d \omega=0,
$$

and; (ii) by the zero-curvature equations for $\mathcal{B}_{n}$,

$$
\omega \wedge \omega=0 .
$$

By these properties, if one formally applies the theorem of Darboux, one can deduce the existence of "Darboux coordinates" $(\mathcal{P}, \mathcal{Q})$ for which

$$
\omega=d \mathcal{P} \wedge d \mathcal{Q}
$$

In fact $\mathcal{L}$ and $\mathcal{M}$ give (and are characterized as) such a pair of functions: 
Proposition 1.3.2. ([1] Proposition2) The $(\mathcal{L}, \mathcal{M})$-pair of the dispersionless KP hierarchy satisfies the exterior differential equation

$$
\omega=d \mathcal{L} \wedge d \mathcal{M}
$$

Conversely, if $\mathcal{L}$ and $\mathcal{M}$ are of the form (1.1.2) and (1.3.2), and satisfy (1.3.10), then $\mathcal{L}$ is a solution of the Lax equations (1.1.1) and $\mathcal{M}$ is a corresponding Orlov function.

This situation is almost parallel to the twistor-theoretical formulation of the self-dual vacuum Einstein equation and hyper-Kähler geometry [16,17].

\section{4. $S$ function and $\tau$ function (free energy).}

Here we introduce two important potentials $S$ and $\log \tau_{\text {dKP }}$ hidden behind the system. Later we shall see that $S$ is the phase function of the WKB analysis of the KP hierarchy and $\log \tau_{\mathrm{dKP}}$ is the leading term of the logarithm of the tau function of the KP hierarchy $\log \tau_{\mathrm{KP}}$ (See $\left.\S 1.7 .4\right)$. Function $\log \tau_{\mathrm{dKP}}(=F)$ is also called "free energy" in the context of matrix models and topological minimal models (See §1.8).

First rewrite (1.3.10) as follows:

$$
d\left(\mathcal{M} d \mathcal{L}+\sum_{n=1}^{\infty} \mathcal{B}_{n} d t_{n}\right)=0 .
$$

This implies the existence of a function $S$ such that

$$
d S=\mathcal{M} d \mathcal{L}+\sum_{n=1}^{\infty} \mathcal{B}_{n} d t_{n},
$$

or, equivalently,

$$
\begin{aligned}
\mathcal{M} & =\left(\frac{\partial S}{\partial \mathcal{L}}\right)_{t \text { fixed }}, \\
\mathcal{B}_{n} & =\left(\frac{\partial S}{\partial t_{n}}\right)_{\mathcal{L}, t_{m}(m \neq n) \text { fixed }} .
\end{aligned}
$$

This potential $S$ is introduced by Krichever in a different manner [7]. His formulation comes from the Whitham theory [23] and, $S$ plays rather a central role than $\mathcal{M}$.

Actually $S$ is not a true potential, but can be written directly in terms of $\mathcal{L}$ and $\mathcal{M}$ as follows. 
Proposition 1.4.1. ([1] Proposition 3) $S$ is given by

$$
S=\sum_{i=1}^{\infty} t_{i} \mathcal{L}^{i}+\sum_{i=1}^{\infty} S_{i} \mathcal{L}^{-i}, \quad S_{i}=-\frac{1}{i} v_{i} .
$$

In particular, $\mathcal{B}_{n}$ can be written explicitly in terms of the Laurent coefficients $S_{i}$ as

$$
\mathcal{B}_{n}=\mathcal{L}^{n}+\sum_{i=1}^{\infty} \frac{\partial S_{i}}{\partial t_{n}} \mathcal{L}^{-i}
$$

Function $\tau_{\mathrm{dKP}}$ is a true potential and defined as follows:

$$
d \log \tau_{\mathrm{dKP}}(t)=\sum_{n=1}^{\infty} v_{n}(t) d t_{n}
$$

Here $d$ denotes the differentiation with respect to $t=\left(t_{1}, t_{2}, \ldots\right)$.

Proposition 1.4.2. ([1] Proposition 6) The right hand side of (1.4.7) is a closed form. Accordingly, there exists a potential $\log \tau_{\mathrm{dKP}}$.

This tau function contains enough information to reproduce any ingredient of the dispersionless KP hierarchy (see [1] $(5.5-7)$ ). Therefore, in principle, one should be able to rewrite the hierarchy in terms of $\log \tau_{\mathrm{dKP}}$. For the case of the ordinary KP hierarchy, the tau function is characterized by the famous Hirota bilinear equations. For

the dispersionless KP hierarchy, we have a series of equations satisfied by $\log \tau_{\mathrm{dKP}}$ (see Appendix B). We conjecture that these equations are enough to characterize $\log \tau_{\mathrm{dKP}}$.

\subsection{Riemann-Hilbert problem (twistor construction).}

The nonlinear graviton (twistor) construction of Penrose [24] for the self-dual vacuum Einstein equation and its hyper-Kähler version [25] can be also extended to the dispersionless KP hierarchy.

Proposition 1.5.1. ([1] Proposition 7.) Let a pair of two functions $(f(k, x), g(k, x))$ and a pair of Laurent series $(\mathcal{L}, \mathcal{M})$ be given. Suppose

(1) $\mathcal{L}$ and $\mathcal{M}$ have the form (1.1.2) and (1.3.2) respectively.

(2) $f$ and $g$ satisfy the canonical Poisson relation $\{f, g\}=1$. 
(3) $\mathcal{P}=f(\mathcal{L}, \mathcal{M})$ and $\mathcal{Q}=g(\mathcal{L}, \mathcal{M})$ are both Taylor series in $k$ :

$$
(f(\mathcal{L}, \mathcal{M}))_{\leq-1}=0, \quad(g(\mathcal{L}, \mathcal{M}))_{\leq-1}=0
$$

Then the pair $(\mathcal{L}, \mathcal{M})$ gives a solution of the dispersionless KP hierarchy. We call $(f, g)$ the twistor data of this solution.

This construction of solutions is a kind of the Riemann-Hilbert decomposition; the area-preserving diffeomorphism $(\mathcal{L}, \mathcal{M}) \mapsto(f(\mathcal{L}, \mathcal{M}), g(\mathcal{L}, \mathcal{M}))$ is decomposed into two maps, $(\mathcal{L}, \mathcal{M}) \mapsto(k, x)$ and $(k, x) \mapsto(\mathcal{P}, \mathcal{Q})$. The former of these maps is rationally extended to $k=\infty$ and the latter to $k=0$.

For a pair $(f, g)$ there exists a unique solution $(\mathcal{L}, \mathcal{M})$ of $(1.5 .1)$ if $(f, g)$ is close enough to the trivial one $(f, g)=(k, x)$. Establishing the existence is a delicate issue in general, and the statement largely depends on the category of functions (formal, holomorphic, etc.). In the following, we always assume the unique solvability of (1.5.1). In contrast to this, the converse statement homds generally.

Proposition 1.5.2. Each solution of the dispersionless KP hierarchy possestes the twistor data, i.e., if $(\mathcal{L}, \mathcal{M})$ is a solution of the dispersionless $K P$ hierarchy, there exists a pair $(f, g)$ which satisfies (2) and (3) of Proposition 1.5.1.

Proof. Let $\exp (\operatorname{ad} \varphi(t))$ be the dressing operator corresponding to $(\mathcal{L}, \mathcal{M})$. Set

$$
f(k, x)=e^{-\operatorname{ad} \varphi(t=0)} k, \quad g(k, x)=e^{-\operatorname{ad} \varphi(t=0)} x .
$$

Obviously the pair $(f, g)$ satisfies the canonical Poisson relation $\{f, g\}=1$. Since $\mathcal{L}(t=0)=\exp (\operatorname{ad} \varphi(t=0)) k$ and $\mathcal{M}(t=0)=\exp (\operatorname{ad} \varphi(t=0)) x$, we have from (1.5.2) the following relations:

$$
\begin{aligned}
& f(\mathcal{L}(t=0), \mathcal{M}(t=0))=e^{\operatorname{ad} \varphi(t=0)} f(k, x)=k, \\
& g(\mathcal{L}(t=0), \mathcal{M}(t=0))=e^{\operatorname{ad} \varphi(t=0)} g(k, x)=x .
\end{aligned}
$$


Now we prove that $f(\mathcal{L}, \mathcal{M})_{\leq-1}=0$. As $\mathcal{L}$ and $\mathcal{M}$ satisfy the Lax equations (1.1.1) and (1.3.3),

$$
\frac{\partial}{\partial t_{n}} f(\mathcal{L}, \mathcal{M})=\left\{\mathcal{B}_{n}, f(\mathcal{L}, \mathcal{M})\right\}
$$

Evaluating (1.5.4) at $t=0$, we see that $\left(\partial f(\mathcal{L}, \mathcal{M}) / \partial t_{n}\right)(t=0)$ does not contain negative powers of $k$ because of (1.5.3). In this way we can prove, by induction, that $(\partial / \partial t)^{\alpha} f(\mathcal{L}, \mathcal{M})(t=0)$, i.e., coefficients of the Taylor expansion at $t=0$ do not contain negative powers of $k$ for any multi-index $\alpha$. Thus we have proved that $f(\mathcal{L}, \mathcal{M})_{\leq-1}=0$. $g(\mathcal{L}, \mathcal{M})_{\leq-1}=0$ is proved in the same way.

This construction of solutions is closely related to the $w_{1+\infty}$-tymmetry of solutions as we shall see in the next section.

1/6. $w_{1+\infty}$ symmetrz.

In the previous section we established a correspondence between a solution $(\mathcal{L}, \mathcal{M})$ and an area-preserving diffeomorphism $(f(k, x), g(k, x))$. Since area-preserving maps form a group $(\operatorname{SDiff}(2)$ in the notation of [1]), we can consider an action of this group on the space of solutions of the dispersionless KP hierarchy. In this section we consider an infinitesimal form of this action, $w_{1+\infty}$-action. We follow the method developed for the self-dual vacuum Einstein equation [26], [17].

Explicitly speaking, we define infinitesimal deformations of $(f, g)$ by a Hamiltonian vector field,

$$
(f, g) \longrightarrow(f, g) \circ \exp (-\varepsilon \operatorname{ad} F)
$$

and the associated deformation

$$
(\mathcal{L}, \mathcal{M}) \longrightarrow(\mathcal{L}(\varepsilon), \mathcal{M}(\varepsilon))
$$

of the $(\mathcal{L}, \mathcal{M})$-pair of the dispersionlest KP hierarchy. Here ad $F$ is regarded as a Hamiltonian vector field

$$
\operatorname{ad} F=\frac{\partial F}{\partial k} \frac{\partial}{\partial x}-\frac{\partial F}{\partial x} \frac{\partial}{\partial k}
$$


and $\varepsilon$ is an infinitesimal parameter. By infinitesimal symmetry we mean the first order coefficient $\delta_{F}(\cdot)$ in $\varepsilon$-expansion:

$$
\begin{aligned}
\mathcal{L}(\varepsilon) & =\mathcal{L}+\varepsilon \delta_{F} \mathcal{L}+O\left(\varepsilon^{2}\right), \\
\mathcal{M}(\varepsilon) & =\mathcal{M}+\varepsilon \delta_{F} \mathcal{M}+O\left(\varepsilon^{2}\right) .
\end{aligned}
$$

By definition, $\delta_{F}$ acts on any function of $\mathcal{L}$ and $\mathcal{M}$ as an abstract derivation,

$$
\delta_{F} G(\mathcal{L}, \mathcal{M})=\frac{\partial G}{\partial \mathcal{L}} \delta_{F} \mathcal{L}+\frac{\partial G}{\partial \mathcal{M}} \delta_{F} \mathcal{M}
$$

while the independent variables of the hierarchy are invariant under this action

$$
\delta_{F} t_{n}=\delta_{F} x=\delta_{F} k=0
$$

(This is a formal way to understand infinitesimal symmetries of differential equations in the language of differential algebras [27]).

A more explicit expression of these infinitesimal symmetries is given by the following propositions.

Proposition 1.6.1. ([1] Proposition 13.) The infinitesimal symmetries of $\mathcal{L}$ and $\mathcal{M}$ are given by

$$
\begin{gathered}
\delta_{F} \mathcal{L}=\left\{F(\mathcal{L}, \mathcal{M})_{\leq-1}, \mathcal{L}\right\} \\
\delta_{F} \mathcal{M}=\left\{F(\mathcal{L}, \mathcal{M})_{\leq-1}, \mathcal{M}\right\} .
\end{gathered}
$$

Proposition 1.6.2. ([1] Proposition 14.) For $i=1,2, \ldots, \delta_{F} v_{i}$ are given by

$$
\delta_{F} v_{i}=-\operatorname{Res} F(\mathcal{L}, \mathcal{M}) d_{k} B_{i}
$$

This is an analogue of the symmetries of Plebanski's key functions of the self-dual Einstein equation [17]. 
Proposition 1.6.3. The infinitesimal symmetry of the dressing function $\delta_{F} \varphi$ is determined by the relation

$$
\nabla_{\delta_{F}, \varphi} \varphi=F(\mathcal{L}, \mathcal{M})_{\leq-1}
$$

or equivalently, by

$$
\delta_{F} \varphi=\left.\frac{\operatorname{ad} \varphi}{e^{\operatorname{ad} \varphi}-1} F(\mathcal{L}, \mathcal{M})_{\leq-1} \underset{\text { def }}{=} \frac{T}{e^{T}-1}\right|_{T=\operatorname{ad} \varphi} F(\mathcal{L}, \mathcal{M})_{\leq-1},
$$

where $T /\left(e^{T}-1\right)$ is understood as a power series of an indeterminate $T$;

$$
\frac{T}{e^{T}-1}=1-\frac{1}{2} T+\cdots
$$

Note that Proposition 1.6.1 is a direct consequence of this proposition.

Proof. Let $(f, g)$ be the twistor data corresponding to $(\mathcal{L}, \mathcal{M})$. By definition the deformed twistor data are given by

$$
\begin{aligned}
\left(f_{\varepsilon}(k, x), g_{\varepsilon}(k, x)\right) & =\left(e^{-\varepsilon \operatorname{ad} F} f(k, x), e^{-\varepsilon \operatorname{ad} F} g(k, x)\right) \\
& =(1-\varepsilon \operatorname{ad} F) \circ(f(k, x), g(k, x))+O\left(\varepsilon^{2}\right) .
\end{aligned}
$$

Let us denote the deformed dressing function by $\varphi_{\varepsilon}(t)=\varphi(t)+\varepsilon \delta_{F} \varphi(t)+O\left(\varepsilon^{2}\right)$. We have (cf. Lemma A.1)

$$
e^{\operatorname{ad} \varphi_{\varepsilon}(t)} e^{\operatorname{ad} \sum t_{n} k^{n}}=\left(1+\varepsilon \operatorname{ad} \nabla_{\delta, \varphi} \varphi\right) \circ e^{\operatorname{ad} \varphi(t)} e^{\operatorname{ad} \sum t_{n} k^{n}}
$$

The Riemann-Hilbert problem (1.5.1) for the deformed solution looks as

$$
\begin{aligned}
& \left(e^{\operatorname{ad} \varphi_{\varepsilon}(t)} e^{\operatorname{ad} \sum t_{n} k^{n}} f_{\varepsilon}(k, x)\right)_{\leq-1}=0 \\
& \left(e^{\operatorname{ad} \varphi_{\varepsilon}(t)} e^{\operatorname{ad} \sum t_{n} k^{n}} g_{\varepsilon}(k, x)\right)_{\leq-1}=0 .
\end{aligned}
$$

Using (1.6.7) and (1.6.8), we have

$$
\begin{aligned}
e^{\operatorname{ad} \varphi_{\varepsilon}(t)} e^{\operatorname{ad} \sum t_{n} k^{n}} f_{\varepsilon}(k, x)= & e^{\operatorname{ad} \varphi(t)} e^{\operatorname{ad} \sum t_{n} k^{n}} f(k, x)+ \\
& +\varepsilon\left(\left\{\nabla_{\delta, \varphi} \varphi, e^{\operatorname{ad} \varphi(t)} e^{\operatorname{ad} \sum t_{n} k^{n}} f(k, x)\right\}\right. \\
& \left.-e^{\operatorname{ad} \varphi(t)} e^{\operatorname{ad} \sum t_{n} k^{n}}\{F, f(k, x)\}\right)+O\left(\varepsilon^{2}\right) \\
= & f(\mathcal{L}, \mathcal{M}) \\
& +\varepsilon\left(\left\{\nabla_{\delta, \varphi} \varphi, f(\mathcal{L}, \mathcal{M})\right\}-\{F(\mathcal{L}, \mathcal{M}), f(\mathcal{L}, \mathcal{M})\}\right) \\
& +O\left(\varepsilon^{2}\right) .
\end{aligned}
$$


We have a similar formula for $g$. Therefore the coefficients of $\varepsilon$ in $(\quad)_{\leq-1}$ on the left hand side of (1.6.9) become

$$
\left\{\nabla_{\delta, \varphi} \varphi-F(\mathcal{L}, \mathcal{M}), f(\mathcal{L}, \mathcal{M})\right\}, \quad\left\{\nabla_{\delta, \varphi} \varphi-F(\mathcal{L}, \mathcal{M}), g(\mathcal{L}, \mathcal{M})\right\}
$$

Hence the following Lemma 1.6.4 proves this Proposition.

Lemma 1.6.4. Suppose that $\mathcal{P}(k, x)$ and $\mathcal{Q}(k, x)$ do not contain negative powers of $k$ and satisfy the canonical Poisson relation $\{\mathcal{P}, \mathcal{Q}\}=1$. If $\mathcal{A}(k, x)$ Poisson-commute with $\mathcal{P}$ and $\mathcal{Q}$ up to positive powers of $k$, i.e.,

$$
(\{\mathcal{A}, \mathcal{P}\})_{\leq-1}=0, \quad(\{\mathcal{A}, \mathcal{Q}\})_{\leq-1}=0
$$

then $\mathcal{A}$ itself does not contain negative powers of $k$.

Proof. We assume $\mathcal{A}_{\geq 0}=0$ and show $\mathcal{A}=0$ under the assumption of the lemma. Lemma 1.6.4 follows immediately from this.

By definition of the Poisson bracket,

$$
\begin{aligned}
& \tilde{\mathcal{P}} \underset{\text { def }}{=}\{\mathcal{A}, \mathcal{P}\}=\frac{\partial \mathcal{A}}{\partial k} \frac{\partial \mathcal{P}}{\partial x}-\frac{\partial \mathcal{A}}{\partial x} \frac{\partial \mathcal{P}}{\partial k}, \\
& \tilde{\mathcal{Q}} \underset{\text { def }}{=}\{\mathcal{A}, \mathcal{Q}\}=\frac{\partial \mathcal{A}}{\partial k} \frac{\partial \mathcal{Q}}{\partial x}-\frac{\partial \mathcal{A}}{\partial x} \frac{\partial \mathcal{Q}}{\partial k} .
\end{aligned}
$$

Regarding this as a system of linear equations for $\partial \mathcal{A} / \partial k, \partial \mathcal{A} / \partial x$, we can rewrite it as

$$
\left(\begin{array}{ll}
\frac{\partial \mathcal{P}}{\partial x} & -\frac{\partial \mathcal{P}}{\partial k} \\
\frac{\partial \mathcal{Q}}{\partial x} & -\frac{\partial \mathcal{Q}}{\partial k}
\end{array}\right)\left(\begin{array}{l}
\frac{\partial \mathcal{A}}{\partial k} \\
\frac{\partial \mathcal{A}}{\partial x}
\end{array}\right)=\left(\begin{array}{c}
\tilde{\mathcal{P}} \\
\tilde{\mathcal{Q}}
\end{array}\right) .
$$

Because of the unimodularity of the matrix in the right hand side, $\{\mathcal{P}, \mathcal{Q}\}=1,(1.6 .12)$ is easily solved as

$$
\left(\begin{array}{l}
\frac{\partial \mathcal{A}}{\partial k} \\
\frac{\partial \mathcal{A}}{\partial x}
\end{array}\right)=\left(\begin{array}{ll}
-\frac{\partial \mathcal{Q}}{\partial k} & \frac{\partial \mathcal{P}}{\partial k} \\
-\frac{\partial \mathcal{Q}}{\partial x} & \frac{\partial \mathcal{P}}{\partial x}
\end{array}\right)\left(\begin{array}{l}
\tilde{\mathcal{P}} \\
\tilde{\mathcal{Q}}
\end{array}\right) .
$$

This means that both $\frac{\partial \mathcal{A}}{\partial k}$ and $\frac{\partial \mathcal{A}}{\partial x}$ do not contain negative powers of $k$, but as we assumed that $\mathcal{A}_{\geq 0}=0$, they must be 0 . Therefore $\mathcal{A}=0$.

These symmetries of the dispersionless KP hierarchy can be lifted up to the tau function as follows. Note that our definition of the tau function has ambiguity of additive constant: this gives rise to an extra term (cocycle) in their commutation relations. 
Proposition 1.6.5. ([1] Proposition 15.) The infinitesimal symmetries $\delta_{F}$ of the $(\mathcal{L}, \mathcal{M})$-pair can be extended to the tau function by defining

$$
\delta_{F} \log \tau=-\operatorname{Res} F^{x}(\mathcal{L}, \mathcal{M}) d_{k} \mathcal{L}
$$

where $F^{x}(k, x)$ is a primitive function of $F(k, x)$ normalized as

$$
F^{x}(k, x) \underset{\operatorname{def}}{=} \int_{0}^{x} F(k, y) d y .
$$

This extended symmetry is compatible with the flows:

$$
\frac{\partial}{\partial t_{n}} \delta_{F} \log \tau=\delta_{F} \frac{\partial \log \tau}{\partial t_{n}}
$$

These symmetries reflect the structure of the $w_{1+\infty}$ algebra as follows.

Proposition 1.6.6. ([1] Proposition 16.) For two generating functions $F_{1}=F_{1}(k, x)$ and $F_{2}=F_{2}(k, x)$, the infinitesimal symmetries $\delta_{F_{1}}$ and $\delta_{F_{2}}$ obey the commutation relations

$$
\begin{aligned}
{\left[\delta_{F_{1}}, \delta_{F_{2}}\right] \log \tau } & =\delta_{\left\{F_{1}, F_{2}\right\}} \log \tau+c\left(F_{1}, F_{2}\right), \\
{\left[\delta_{F_{1}}, \delta_{F_{2}}\right] \mathcal{K} } & =\delta_{\left\{F_{1}, F_{2}\right\}} \mathcal{K}, \quad(\mathcal{K}=\mathcal{L}, \mathcal{M}), \\
{\left[\delta_{F_{1}}, \delta_{F_{2}}\right] \varphi } & =\delta_{\left\{F_{1}, F_{2}\right\}} \varphi
\end{aligned}
$$

where

$$
c\left(F_{1}, F_{2}\right) \underset{\text { def }}{=} \operatorname{Res} F_{1}(k, 0) d F_{2}(k, 0)
$$

which is a cocycle of the $w_{1+\infty}$ algebra.

Proof. Equations (1.6.17) and (1.6.18) are proved in [1]. The statement for the dressing function (1.6.19) is an immediate consequence of (1.3.4), (1.6.17) and the compatibility of the deformations with the time flows (1.6.16). 


\subsection{Quasi-classical limit of KP hierarchy.}

In this section we change our viewpoint and describe the results in the previous sections as quasi-classical limit. We take a slightly different way from [3] but the principle is the same. Namely, we intsoduce a "Planck constant" $\hbar$ into the oreinary KP hierarchy and take the limit $\hbar \rightarrow 0$. Leading terms in this limit recover the dispersionless KP hierarchy. The contents of the following subsections $1.7 .1-6$ are organized to correspond to the contents of $\S \S 1.1-6$, respectively.

1.7.1 Lax formalism. We define the KP hierarchy in the presence of $\hbar$ as the Lax representation

$$
\hbar \frac{\partial L}{\partial t_{n}}=\left[B_{n}, L\right], \quad B_{n}=\left(L^{n}\right)_{\geq 0}, \quad n=1,2, \ldots
$$

where $L$, the counterpart of $\mathcal{L}$, is a micro-differential operator (in $x$ ),

$$
L=\hbar \partial+\sum_{n=1}^{\infty} u_{n+1}(\hbar, t)(\hbar \partial)^{-n}, \quad \partial=\partial / \partial x
$$

and "( $) \geq 0$ " now stands for the projection onto a differential operator dropping negative powers of $\partial$. The coefficients $u_{n}(\hbar, t)$ of $L$ are assumed to be regular with respect to $\hbar$. This means that they have such an asymptotic form as $u_{n}(\hbar, t)=u_{n}^{0}(t)+O(\hbar)$ around $\hbar=0$. Every ingredient in the KP hierarchy is now to depend on $\hbar$. For later discussion, we now define the "order" and the "principal symbol" of micro-differential operators with $\hbar$ as follows.

Definition 1.7.1.

$$
\operatorname{ord}^{\hbar}\left(\sum a_{n, m}(t) \hbar^{n} \partial^{m}\right) \underset{\text { def }}{=} \max \left\{m-n \mid b_{n, m}(t) \neq 0\right\}
$$

In particular, $\operatorname{ord}^{\hbar}(\hbar)=-1$, $\operatorname{ord}^{\hbar}(\partial)=1$. The principal symbol (resp. the symbol of order $l$ ) of a micro-differential operator $A=\sum a_{n, m} \hbar^{n} \partial^{m}$ is

$$
\begin{gathered}
\sigma^{\hbar}(A) \underset{\text { def }}{=} \hbar^{-\operatorname{ord}^{\hbar}(A)} \sum_{m-n=\operatorname{ord}^{\hbar}(A *} a_{n, m} k^{m} \\
\text { (resp. } \sigma_{m}^{\hbar}(A) \underset{\text { def }}{=} \hbar^{-l} \\
\left.\sum_{m-n=l} a_{n, m} k^{m}\right) .
\end{gathered}
$$


For example, the condition which we imposed on the coefficients $u_{n}(\hbar, t)$ can be

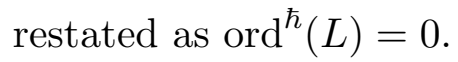

Remark 1.7.2. This "order" coincides with the order of an micro-differential operator if we formally replace $\hbar$ with $\partial_{t_{0}}^{-1}$, where $t_{0}$ is an extra variable. In fact, naively extending (1.7.1) to $n=0$, we can introduce the time variable $t_{0}$ on which nothing depends.

Following facts are easily proved.

Lemma 1.7.3. Let $P_{i}(i=1,2)$ be two micro-differential operators of finite order: $\operatorname{ord}^{\hbar}\left(P_{i}\right)=l_{i}$

(1) $\operatorname{ord}^{\hbar}\left(P_{1} P_{2}\right)=l_{1}+l_{2}$ and

$$
\sigma^{\hbar}\left(P_{1} P_{2}\right)=\sigma^{\hbar}\left(P_{1}\right) \sigma^{\hbar}\left(P_{2}\right)
$$

(2) $\operatorname{ord}^{\hbar}\left(\left[P_{1}, P_{2}\right]\right) \leqq l_{1}+l_{2}-1$ and

$$
\sigma_{l_{1}+l_{2}-1}^{\hbar}\left(\left[P_{1}, P_{2}\right]\right)=\hbar\left\{\sigma^{\hbar}\left(P_{1}\right), \sigma^{\hbar}\left(P_{2}\right)\right\}
$$

where $\{$,$\} is the Poisson bracket defined by (1.1.3).$

Taking the principal symbol of (1.7.1) and using Lemma 1.7.3, we obtain

Proposition 1.7.4. $\mathcal{L}=\sigma^{\hbar}(L)$ is a solution of the dispersionlets KP hierarchy (1.1.1*, and $\mathcal{B}_{n}=\sigma^{\hbar}\left(B_{n}\right)$.

1.7.2 Dressing operators. In order to find a counterpart of the dressing function $\varphi$, we need a more refined construction of the dressing operator $W$ of the ordinary KP hierarchy in our context.

Proposition 1.7.5. There is a dressing operator $W$,

$$
\begin{aligned}
L & =W(\hbar \partial) W^{-1}, \\
\hbar \frac{\partial W}{\partial t_{n}} W^{-1} & =-\left(W(\hbar \partial)^{n} W^{-1}\right)_{\leq-1},
\end{aligned}
$$


of the form

$$
\begin{gathered}
W=\exp \left(\hbar^{-1} X(\hbar, t, \partial)\right) \\
X(\hbar, t, \partial)=\sum_{n=1}^{\infty} \chi_{n}(\hbar, t)(\hbar \partial)^{-n}, \quad \operatorname{ord}^{\hbar}(X(\hbar, t, \partial))=0 .
\end{gathered}
$$

Conversely, if $W$ of the form (1.7.6) satisfies (1.7.5), then $L=W(\hbar \partial) W^{-1}$ is a soluiton of the Lax equations (1.7.1).

Proof. The proof is mostly parallel to the proof of Proposition 1.2.1. According to Lemma 1.7.3, the linear space of micro-differential operators of order not greater than $1, \mathcal{E}_{1}=\left\{P\right.$ : micro-differential operator $\left.\mid \operatorname{ord}^{\hbar}(P) \leqq 1\right\}$, is closed under the commutator and thus is a Lie algebra. Therefore, replacing $\{\},, \varphi, k$ by $[],, X / \hbar$, $\hbar \partial$, respectively, we can carry over the proof of Proposition 1.2.1 to this case. Here $\operatorname{ad}(X / \hbar)(\cdot)=[X / \hbar, \cdot]$.

The dressing function $\varphi$ of the dispersionless KP hierarchy and $W$ are connected as follows:

Corollary 1.7.6. $\sigma^{\hbar}(X)$ gives a dressing function $\varphi(t)$ of $\mathcal{L}=\sigma^{\hbar}(L)$ in the sense of §1.2. Conversely, if $\varphi(t)$ is a dressing function of a solution $\mathcal{L}$ of the dispersionless $K P$ hierarchy, there exists a solution of the KP hierarchy $L$ and a corresponding dressing operator $W=\exp \left(\hbar^{-1} X\right)$ such that $\mathcal{L}=\sigma^{\hbar}(L), \varphi(t)=\sigma^{\hbar}(X)$.

Proof. The first statement is obvious from the above proof. The second statement is proved by the unique solvability of the initial value problem of the KP hierarchy: suppose $\varphi$ and $\mathcal{L}$ are given. Then there exists a micro-differential operator $X_{0}(\hbar, x, \hbar \partial)$ such that $\sigma^{\hbar}\left(X_{0}\right)=\varphi\left(t_{1}=x, t_{2}=t_{3}=\ldots=0\right)$. The solution of the KP hierarchy $L$ and a corresponding dressing operator $W$ with the initial value $W\left(t_{1}=x, t_{2}=t_{3}=\right.$ $\ldots=0)=\exp \left(\hbar^{-1} X_{0}\right)$ is the desired $W$ and $L$.

Regarding $\hbar=\partial_{t_{0}}^{-1}$ as in Remark 1.7.2, $\operatorname{Ad}\left(W \exp \left(\sum t_{n}(\hbar \partial)^{n}\right)\right)$ is nothing but the "quantized canonical transformation" [19] corresponding to the canonical transformation $\exp (\operatorname{ad} \varphi) \exp \left(\operatorname{ad} \sum t_{n} k^{n}\right)$ 
1.7.3 Orlov operator. The Orlov operator $M$ is defined by

$$
\begin{aligned}
M & =W\left(\sum_{n=1}^{\infty} n t_{n}(\hbar \partial)^{n-1}+x\right) W^{-1}=\operatorname{Ad}\left(W \exp \left(\hbar^{-1} t(\hbar \partial)\right)\right) x \\
& =\sum_{n=1}^{\infty} n t_{n} L^{n-1}+x+\sum_{n=1}^{\infty} v_{n}(\hbar, t) L^{-n-1},
\end{aligned}
$$

where $t(\hbar \partial)=\sum_{n=1}^{\infty} t_{n}(\hbar \partial)^{n}$. The proof of the gollowing facts is analogous to that of Proposition 1.3.1.

\section{Proposition 1.7.7.}

(1) $\operatorname{ord}^{\hbar}(M)=0$;

(2) $[L, M]=\hbar$;

(3) $M$ satisfies the Lax equations,

$$
\hbar \frac{\partial M}{\partial t_{n}}=\left[B_{n}, M\right], \quad n=1,2, \ldots
$$

where $B_{n}$ are defined in (1.7.1).

Conversely, if $M$ of the form

$$
M=\sum_{n=1}^{\infty} n t_{n} L^{n-1}+x+\sum_{n=1}^{\infty} v_{n}(\hbar, t) L^{-n-1}
$$

satisfies (1) - (3) above, then there exists a unique dressing operator $W$ such that $M$ is expressed as (1.7.7).

Corollary 1.7.8. $\mathcal{M}=\sigma^{\hbar}(M)$ is the Orlov function of the dispersionless KP hierarchy corresponding to $\mathcal{L}=\sigma^{\hbar}(L)$.

1.7.4. S function and $\tau$ function (free energy). The $S$ function introduced in $\S 1.4$ appears as the phase function of the WKB analysis of the system. As usual we define the Baker-Akhiezer function (or the wave function in the terminology of [15]) by

$$
\Psi(\hbar, t, \lambda)=W(\hbar, t, \partial) \exp \left(\hbar^{-1} t(\lambda)\right), \quad t(\lambda) \underset{\text { def }}{=} \sum_{n=1}^{\infty} t_{n} \lambda^{n}
$$


that satisfies the linear equations

$$
\lambda \Psi=L \Psi, \quad \hbar \frac{\partial \Psi}{\partial \lambda}=M \Psi, \quad \hbar \frac{\partial \Psi}{\partial t_{n}}=B_{n} \Psi
$$

due to (1.7.5), (1.7.7). Since $\hbar \partial \exp \left(\hbar^{-1} t(\lambda)\right)=\lambda \exp \left(\hbar^{-1} t(\lambda)\right)$, we have

$$
\Psi(\hbar, t, \lambda)=\left(1+O\left(\lambda^{-1}\right)\right) \exp \left(\hbar^{-1} t(\lambda)\right), \quad \lambda \rightarrow \infty, \hbar \rightarrow 0 .
$$

More precisely, Proposition 1.7.7 (1) and equations (1.7.10) imply the following

Proposition 1.7.9. The Baker-Akhiezer function has the following WKB asymptotic expansion as $\hbar \rightarrow 0:$

$$
\Psi(\hbar, t, \lambda)=\exp \left(\hbar^{-1} S(t, \lambda)+O\left(\hbar^{0}\right)\right)
$$

where $S(t, \lambda)=\sum_{n=1}^{\infty} t_{n} \lambda^{n}+\sum_{n=1}^{\infty} S_{n}(t) \lambda^{-n}$.

We can further pursue the WKB analysis, regarding $S(t, \lambda)$ as the phase function. The Hamilton-Jacobi (or eikonal) equations corresponding to (1.7.10) are

$$
\begin{aligned}
& \lambda=\frac{\partial S(t, \lambda)}{\partial x}+\sum_{n=1}^{\infty} u_{n+1}^{0}(t)\left(\frac{\partial S(t, \lambda)}{\partial x}\right)^{-n}=\left.\sigma^{\hbar}(L)\right|_{k=\frac{\partial S(t, \lambda)}{\partial x}} \\
& d S(t, \lambda)=\mathcal{M}(t, \lambda) d \lambda+\sum_{n=1}^{\infty} \mathcal{B}_{n}(t, \lambda) d t_{n}
\end{aligned}
$$

where

$$
\begin{aligned}
& \mathcal{M}(t, \lambda)=\left.\sigma^{\hbar}(M)\right|_{\mathcal{L}=\lambda}=\sum_{n=1}^{\infty} n t_{n} \lambda^{n-1}+\sum_{n=1}^{\infty} v_{n}^{0}(t) \lambda^{-n-1}, \\
& \mathcal{B}_{n}(t, \lambda)=\left.\sigma^{\hbar}\left(B_{n}\right)\right|_{k=\frac{\partial S(t, \lambda)}{\partial x}}=\left(\frac{\partial S(t, \lambda)}{\partial x}\right)^{n}+\sum_{i=0}^{n-2} b_{n, i}^{0}(t)\left(\frac{\partial S(t, \lambda)}{\partial x}\right)^{i},
\end{aligned}
$$

and the coefficients $b_{n, i}^{0}(t)$, like $u_{n}^{0}(t)$ and $v_{n}^{0}(t)$, are the leading $\left(\hbar^{0}\right)$ term in $\hbar$-expansion of the coefficients $b_{n, i}(\hbar, t)$ of $B_{n}=(\hbar \partial)^{n}+\sum_{i=0}^{n-2} b_{n, i}(\hbar, t)(\hbar \partial)^{i}$.

Thus we obtain 
Proposition 1.7.10. Under the Legendre-type transformation $(t, \lambda) \rightarrow(t, k)$ given by

$$
k=\frac{\partial S(t, \lambda)}{\partial x} \quad\left(x=t_{1}\right),
$$

the spectral parameter $\lambda$ turns into the $\mathcal{L}$-function of the dispersionless $K P$ hierarchy,

$$
\lambda=\mathcal{L}(t, k)=k+\sum_{n=1}^{\infty} u_{n+1}^{0}\left(t * k^{-n}\right.
$$

whereas $S(t, \lambda)$ becomes the corresponding $S$ function,

$$
S(t, \lambda)=S=\sum_{n=1}^{\infty} t_{n} \mathcal{L}^{n}+\sum_{n=1}^{\infty} S_{n} \mathcal{L}^{-n} .
$$

The tau fvnction $\tau(\hbar, t)$ is by definition a fvnctjon that reproducet the Baker-Dkhiezer function as

$$
\begin{aligned}
& \Psi(\hbar, t)=\frac{\tau\left(\hbar, t-\hbar\left[\lambda^{-1}\right]\right)}{\tau(\hbar, t)} \exp \left(\hbar^{-1} t(\lambda)\right), \\
& {\left[\lambda^{-1}\right]=\left(\frac{1}{\lambda}, \frac{1}{2 \lambda^{2}}, \frac{1}{3 \lambda^{3}}, \ldots\right) .}
\end{aligned}
$$

One can easily show that the tau function $\tau(\hbar, t)$ must behave as

$$
\tau(\hbar, t)=\exp \left[\hbar^{-2} F(t)+O\left(\hbar^{-1}\right)\right] \quad(\hbar \rightarrow 0)
$$

so as to reproduce the WKB asymptotic form of the Baker-Akhiezer function (1.7.12). Combining (1.7.17) and the second equation of (1.7.10), one can further prove that the function $F(t)$ satisfies the equations

$$
\frac{\partial F(t)}{\partial t_{n}}=v_{n}^{0}(t), \quad n=1,2, \ldots
$$

Therefore $F(t)$ can be identified with $\log \tau_{\mathrm{dKP}}$.

1.7.5 Riemann-Hilbert problem (twistor construction). The twistor construction of $\S 1.5$ can be extended to the construction of solutions to the ordinary KP hierarchy under the present setting. The parameter $\hbar$ turns out to play a rather crucial role. 
Proposition 1.7.11. Suppose that

$$
\begin{aligned}
& f(\hbar, x, \hbar \partial)=\sum_{n \in \mathbb{Z}} f_{n}(\hbar, x)(\hbar \partial)^{n} \\
& g(\hbar, x, \hbar \partial)=\sum_{n \in \mathbb{Z}} g_{n}(\hbar, x)(\hbar \partial)^{n}
\end{aligned}
$$

are micro-differential operators of 0 -th order (in the sense of (1.7.3)), $\operatorname{ord}^{\hbar} f=$ $\operatorname{ord}^{\hbar} g=0$, and that they satisfy the canonical commutation relation $[f, g]=\hbar$. Assume that micro-differential operators $L$ of the form (1.7.2) and $M$ of the form $M=\sum_{n=1}^{\infty} n t_{n} L^{n-1}+x+\sum_{n=1}^{\infty} v_{n}(\hbar, t) L^{-n-1}$ are given and that $\operatorname{ord}^{\hbar} L=\operatorname{ord}^{\hbar} M=0$, $[L, M]=\hbar$.

Then, if $f(\hbar, M, L)$ and $g(\hbar, M, L)$ are both differential operators, i.e.,

$$
(f(\hbar, M, L))_{\leq-1}=(g(\hbar, M, L))_{\leq-1}=0
$$

$L$ is a solution of the KP hierarchy, and $M$ is the corresponding Orlov operator.

Proof. From the assumption on the form of $L$ and $M$, it is easy to prove that there exists a micro-differential operator of the form

$$
X(\hbar, t, \partial)=\sum_{n=1}^{\infty} \chi_{n}(\hbar, t)(\hbar \partial)^{-n}, \quad \operatorname{ord}^{\hbar}(X(\hbar, t, \partial))=0
$$

which satisfies

$$
\begin{aligned}
L(\hbar, t, \hbar \partial) & =\operatorname{Ad}(W)(\hbar \partial) \\
M(\hbar, t, \hbar \partial) & =\operatorname{Ad}\left(W \exp \left(\hbar^{-1} t(\hbar \partial)\right)\right) x
\end{aligned}
$$

where $W(\hbar, t, \hbar \partial)=\exp \left(\hbar^{-1} X(\hbar, t, \hbar \partial)\right), t(\hbar \partial)=\sum_{n=1}^{\infty} t_{n}(\hbar \partial)^{n}$. Put $P=f(\hbar, M, L)$, $Q=g(\hbar, M, L)$. The assumption (1.7.20) of the proposition says that $P$ and $Q$ are differential operators

$$
P_{\leq-1}=Q_{\leq-1}=0
$$

Differentiating the equation

$$
P=\operatorname{Ad}\left(W \exp \left(\hbar^{-1} t(\hbar \partial)\right)\right) f(\hbar, x, \hbar \partial)
$$


we obtain

$$
\hbar \frac{\partial P}{\partial t_{n}}=\left[\hbar \frac{\partial W}{\partial t_{n}} W^{-1}+W(\hbar \partial)^{n} W^{-1}, P\right]
$$

and similarly,

$$
\hbar \frac{\partial Q}{\partial t_{n}}=\left[\hbar \frac{\partial W}{\partial t_{n}} W^{-1}+W(\hbar \partial)^{n} W^{-1}, Q\right]
$$

Put $A=\hbar \frac{\partial W}{\partial t_{n}} W^{-1}+W(\hbar \partial)^{n} W^{-1}$. The statement of the proposition is equivalent to $A_{\leq-1}=0$, since this means

$$
\hbar \frac{\partial W}{\partial t_{n}}=-\left(L^{n}\right)_{\leq-1} W
$$

and it is well known that this is an alternative expression of the KP hierarchy in terms of the dressing operator: $L=W(\hbar \partial) W^{-1}$.

Now let us suppose that $A_{-} \underset{\text { def }}{=} A_{\leq-1} \neq 0$. Putting $A_{+} \underset{\text { def }}{=} A_{\geq 0},(1.7 .21)$ and (1.7.22) imply that

$$
\begin{aligned}
& {\left[A_{-}, P\right]=\hbar \frac{\partial P}{\partial t_{n}}-\left[A_{+}, P\right],} \\
& {\left[A_{-}, Q\right]=\hbar \frac{\partial Q}{\partial t_{n}}-\left[A_{+}, Q\right] .}
\end{aligned}
$$

Due to $\left(1.7 .20^{\prime}\right)$, the right hand sides of (1.7.23) are differential operators. It follows from the assumption $A_{-} \neq 0$ that $\mathcal{A}_{-} \underset{\text { def }}{=} \sigma^{\hbar}\left(A_{-}\right) \neq 0$. Thus taking the principal symbol of (1.7.23), we have

$$
\left(\left\{\mathcal{A}_{-}, \mathcal{P}\right\}\right)_{\leq-1}=\left(\left\{\mathcal{A}_{-}, \mathcal{Q}\right\}\right)_{\leq-1}=0
$$

where $\mathcal{P}=\sigma^{\hbar}(P), \mathcal{Q}=\sigma^{\hbar}(Q)$. Moreover, the canonical commutation relation $[P, Q]=\hbar$ leads to the canonical Poisson relation $\{\mathcal{P}, \mathcal{Q}\}=1$. Hence according to Lemma 1.6.4, $\mathcal{A}_{-}=0$. This is a contradiction.

As in the case of the dispersionless KP hierarchy, we call the pair $(f, g)$ above the twistor data.

Proposition 1.7.12. Any solution of the KP hierarchy possesses a twistor data, i.e., if $(L, M)$ is a soluiton of the KP hierarchy in the presence of $\hbar$, there exists a pair $(f, g)$ 
which satisfies the canonical commutation relation $[f, g]=\hbar$ and (1.7.20). In addition, we can choose $f$ and $g$ such that $f$ is a first order micro-differential operator with respect to $\partial_{x}$ and $g$ a zeroth order micro-differential operator.

Proof. Let $W=\exp \left(\hbar^{-1} X\right), \operatorname{ord}^{\hbar}(X)=0$, be the dressing operator chosen in Proposition 1.7.5. Set

$$
f(\hbar, x, \hbar \partial) \underset{\text { def }}{=} W(t=0)^{-1}(\hbar \partial) W(t=0), \quad g(\hbar, x, \hbar \partial) \underset{\text { def }}{=} W(t=0)^{-1} x W(t=0) .
$$

Obviously, $[f, g]=\hbar$ and

$$
\begin{gathered}
\left.f(\hbar, M, L)\right|_{t=0}=W(t=0) f(\hbar, x, \hbar \partial) W(t=0)^{-1}=\hbar \partial, \\
\left.g(\hbar, M, L)\right|_{t=0}=W(t=0) g(\hbar, x, \hbar \partial) W(t=0)^{-1}=x
\end{gathered}
$$

do not contain negative powers of $\hbar \partial$. Due to (1.7.5),

$$
\begin{aligned}
\hbar \frac{\partial}{\partial t_{n}} f(\hbar, M, L) & =\left[\hbar \frac{\partial W}{\partial t_{n}} W^{-1}+W(\hbar \partial)^{n} W^{-1}, f(\hbar, M, L)\right] \\
& =\left[B_{n}, f(\hbar, M, L)\right]
\end{aligned}
$$

where $B_{n}=\left(L^{n}\right)_{\geq 0}$. Proceeding in this manner, we can prove by induction that all the coefficients of the Taylor expansion of $f(\hbar, M, L)$ with respect to $t$ are differential operators. The proof of $(g(\hbar, M, L))_{\leq-1}=0$ is the same.

Needless to say, the principal symbols $\sigma^{\hbar}(f)$ and $\sigma^{\hbar}(g)$ give the twistor data for the $(\mathcal{L}, \mathcal{M})$-pair $\mathcal{L}=\sigma^{\hbar}(L), \mathcal{M}=\sigma^{\hbar}(M)$ of the dispersionless KP hierarchy.

The uniqueness of the solution to (1.7.20) is true in the following infinitesimal sense.

Proposition 1.7.13. Suppose that (1.7.20) has a one-parameter family of solutions $(L(\varepsilon), M(\varepsilon))$ regular in a neighborhood of $\varepsilon=0$ :

$$
(f(\hbar, M(\varepsilon), L(\varepsilon)))_{\leq-1}=(g(\hbar, M(\varepsilon), L(\varepsilon)))_{\leq-1}=0 .
$$

Then $L(\varepsilon)=L(0), M(\varepsilon)=M(0)$ for any $\varepsilon$. 
Proof. We can choose $W=W(\varepsilon)$ in the proof of Proposition 1.7.11 so that $W(\varepsilon)$ depends on $\varepsilon$ regularly. Set $P(\varepsilon)=f(\hbar, M(\varepsilon), L(\varepsilon)), Q(\varepsilon)=g(\hbar, M(\varepsilon), L(\varepsilon))$. Since they are differential operators, both hand sides of

$$
\begin{aligned}
& \frac{\partial P}{\partial \varepsilon}=\left[\frac{\partial W}{\partial \varepsilon} W^{-1}, P\right], \\
& \frac{\partial Q}{\partial \varepsilon}=\left[\frac{\partial W}{\partial \varepsilon} W^{-1}, Q\right]
\end{aligned}
$$

are also differential operators. Thus, as in the proof of Proposition 1.7.11, $(\partial W / \partial \varepsilon) W^{-1}$ should not contain negative powers of $\partial$, hence $\partial W / \partial \varepsilon=0$.

1.7.6. $W_{1+\infty}$ symmetry. We now turn to the issue of $W_{1+\infty}$ and $w_{1+\infty}$ symmetries, studied in [3]. There are two possible ways to deform solutions of the KP hierarchy in our context; one is to deform the twistor data as in $\S 1.6$, and the other is to consider the action of the transformation group as in [3].

First we deform the twistor data $(f(\hbar, x, \hbar \partial), g(\hbar, x, \hbar \partial))$ of a solution of the KP hierarchy described in $§ 1.7 .5$, composing it to an infinitesimal quantized contact transformation $(\hbar \partial, x) \mapsto\left(\operatorname{Ad}\left(e^{-\varepsilon Y}\right) \hbar \partial, \operatorname{Ad}\left(e^{-\varepsilon Y}\right) x\right)$.

Proposition 1.7.14. The above symetries of solutions induced by a micro-differential operator $Y(\hbar, x, \hbar \partial)$ of order not greater than $1, \operatorname{ord}^{\hbar}(Y) \leqq 1$, has explicit form as:

$$
\begin{aligned}
\delta_{Y} L & =\left[(Y(\hbar, M, L))_{\leq-1}, L\right], \\
\delta_{Y} M & =\left[(Y(\hbar, M, L))_{\leq-1}, M\right], \\
\delta_{Y} W \cdot W^{-1} & =(Y(\hbar, M, L))_{\leq-1} .
\end{aligned}
$$

Proof is the same as that in $\S 1.6$, if one replaces the Poisson bracket by the commutator, but we give here the proof of the last statement for later discussion. Let us denote the deformed twistor data by $\left(f_{\varepsilon}, g_{\varepsilon}\right)$, and the corresponding dressing operator by $W_{\varepsilon}$. Define

$$
\begin{aligned}
(P, Q) & :=\left(\operatorname{Ad}\left(W e^{\hbar^{-1} t(\hbar \partial)}\right) f, \operatorname{Ad}\left(W e^{\hbar^{-1} t(\hbar \partial)}\right) g\right), \\
\left(P_{\varepsilon}, Q_{\varepsilon}\right) & :=\left(\operatorname{Ad}\left(W_{\varepsilon} e^{\hbar^{-1} t(\hbar \partial)}\right) f_{\varepsilon}, \operatorname{Ad}\left(W_{\varepsilon} e^{\hbar^{-1} t(\hbar \partial)}\right) g_{\varepsilon}\right),
\end{aligned}
$$


which are, by construction, all differential operators. As $f_{\varepsilon}=\operatorname{Ad}(\exp (-\varepsilon Y)) f$, we have

$$
\begin{aligned}
P_{\varepsilon} & =\operatorname{Ad}\left(W_{\varepsilon} e^{\hbar^{-1} t(\hbar \partial)} \exp (-\varepsilon Y) e^{-\hbar^{-1} t(\hbar \partial)} W^{-1}\right) P, \\
Q_{\varepsilon} & =\operatorname{Ad}\left(W_{\varepsilon} e^{\hbar^{-1} t(\hbar \partial)} \exp (-\varepsilon Y) e^{-\hbar^{-1} t(\hbar \partial)} W^{-1}\right) Q .
\end{aligned}
$$

Hence, by the same argument as the proof of Proposition 1.7.13,

$$
W_{\varepsilon} e^{\hbar^{-1} t(\hbar \partial)} \exp (-\varepsilon Y) e^{-\hbar^{-1} t(\hbar \partial)} W^{-1}
$$

is a differential operator. Therefore $W_{\varepsilon}$ is given by the decomposition

$$
W e^{\hbar^{-1} t(\hbar \partial)} e^{\varepsilon Y} e^{-\hbar^{-1} t(\hbar \partial)}=V_{\varepsilon} W_{\varepsilon}
$$

where $V_{\varepsilon}$ is a differential operator. Differentiating both hand sides of (1.7.27) with respect to $\varepsilon$, it is easy to see that

$$
\begin{gathered}
W_{\varepsilon}=\exp \left(\varepsilon\left(W e^{\hbar^{-1} t(\hbar \partial)} Y e^{-\hbar^{-1} t(\hbar \partial)} W^{-1}\right)_{\leq-1}+O\left(\varepsilon^{2}\right)\right) W, \\
V_{\varepsilon}=\exp \left(\varepsilon\left(W e^{\hbar^{-1} t(\hbar \partial)} Y e^{-\hbar^{-1} t(\hbar \partial)} W^{-1}\right)_{\geq 0}+O\left(\varepsilon^{2}\right)\right),
\end{gathered}
$$

which proves the proposition.

Since both hand sides of (1.7.27) are in the Lie algebra $\mathcal{E}_{1}=\left\{\operatorname{ord}^{\hbar}(Y) \leqq 1\right\}$, we can take the symbol of order 0 of the first and second equations of (1.7.27) and the symbol of order 1 of the last equation. The result gives the infinitesimal deformation of a solution of the dispersionless KP hierarchy in $\S 1.6(F(k, x)$ therein is now given by $\left.\hbar \sigma_{1}^{\hbar}(Y(\hbar, x, \hbar \partial))\right)$.

On the other hand we presented in [3] another approach to the symmetries as follows. A field theoretical interpretation of the KP hierarchy was established by Date et al. [15]. In [3] we studied quasi-classical limit of $W_{1+\infty}$ symmetries in terms of vertex operators and free fermions introduced by them: we first modify their bosonized vertex operator $Z(\tilde{\lambda}, \lambda)$ by rescaling $t_{n} \rightarrow \hbar^{-1} t_{n}, \partial / \partial t_{n} \rightarrow \hbar \partial / \partial t_{n}$ as:

$$
\begin{gathered}
Z(\hbar, \tilde{\lambda}, \lambda)=\frac{\exp \left[\hbar^{-1} t(\tilde{\lambda})-\hbar^{-1} t(\lambda)\right] \exp \left[-\hbar \tilde{\partial}\left(\tilde{\lambda}^{-1}\right)+\hbar \tilde{\partial}\left(\lambda^{-1}\right)\right]-1}{\tilde{\lambda}-\lambda}, \\
t(\lambda)=\sum_{n=1}^{\infty} t_{n} \lambda^{n}, \quad \tilde{\partial}\left(\lambda^{-1}\right)=\sum_{n=1}^{\infty} \lambda^{-n} \frac{1}{n} \frac{\partial}{\partial t_{n}} .
\end{gathered}
$$


This rescaled vertex operator is a generating function of infinitesimal symmetries of the KP hierarchy with $\hbar$ inserted, acting on the tau function as $\tau \rightarrow \tau+\varepsilon Z(\hbar, \tilde{\lambda}, \lambda) \tau$. If one expands this two-parameter family of symmetries into Fourier modes along the double loop $|\tilde{\lambda}|=|\lambda|=$ const., the outcome are generators of $\mathfrak{g l}(\infty)$ symmetries [15]. If one first expands this vertex operator into Taylor series along the diagonal $\tilde{\lambda}=\lambda$, and further into Fourier modes along the loop $|\lambda|=$ const., one obtains a set of generators of $W_{1+\infty}$ symmetries, $W_{n}^{(\ell)}(\hbar)(\ell \geqq 1, n \in \mathbb{Z})$. Explicitly, these generators are given by

$$
W^{(\ell)}(\hbar, \lambda)=\sum_{n=-\infty}^{\infty} W_{n}^{(\ell)}(\hbar) \lambda^{-n-\ell}=\left.\left(\frac{\partial}{\partial \tilde{\lambda}}\right)^{\ell-1} Z(\hbar, \tilde{\lambda}, \lambda)\right|_{\tilde{\lambda}=\lambda} .
$$

Each of $W_{n}^{(\ell)}(\hbar)$ acts on the tau function as a differential operator of finite order. The $w_{1+\infty}$ symmetries are quasi-classical limit of these $W_{1+\infty}$ symmetries defined by

$$
w_{n}^{(\ell)} \log \tau_{\mathrm{dKP}}=\lim _{\hbar \rightarrow 0} \tau(\hbar, t)^{-1} \hbar^{\ell} W_{n}^{(\ell)}(\hbar) \tau(\hbar, t)
$$

This limit can be calculated more explicitly as:

$$
w_{n}^{(\ell)} \log \tau_{\mathrm{dKP}}=\frac{1}{\ell} \operatorname{Res} \mathcal{M}^{\ell} \mathcal{L}^{n+\ell-1} d_{k} \mathcal{L}
$$

These are nothing but the generators of $w_{1+\infty}$ symmetries in $§ 1.6$, i.e., the symmetries generated by $F(k, x)=-x^{\ell-1} k^{n+\ell-1}$.

The two constructions of symmetries due to Proposition 1.7.14 for $L, M, W$ and due to (1.7.32) for $\log \tau_{\mathrm{dKP}}$ are, actually, closely related. To see this fact, it is convenient to use the fermionic and the wedge representation.

Let us recall briefly how the dressing operator determines the tau function in the theory of the ordinary $(\hbar=1) \mathrm{KP}$ hierarchy. According to [13], [14] and [15], the dressing operator is in one-to-one correspondence with the point in the universal Grassmann manifold $U G M$. When the motion of a point $U(t) \in U G M$ obeys the KP hierarchy, one of the Plücker coordinate gives the tau function. To make this correspondence explicit, we prepare notations. We denote the ring of micro-differential operators and 
the ring of differential operators of one variable $x$ by $\mathcal{E}$ and $\mathcal{D}$ respectively. For the moment we take $\mathbb{C}$ as the constant ring of $\mathcal{E}$ and $\mathcal{D}$. Define the $\mathcal{E}$-module $\mathcal{V}$ and the $\mathcal{D}$-module $V^{\phi}$ generated by the delta function $\delta(x)$ by

$$
\mathcal{V}:=\mathcal{E} / \mathcal{E} x, \quad V^{\phi}:=\mathcal{D} / \mathcal{D} x
$$

The $\mathcal{D}$-module $V^{\phi}$ is naturally regarded as a sub- $\mathcal{D}$-module of $\mathcal{V}$. We take the basis $\left\{e_{\nu}=\partial^{-1-\nu} \bmod \mathcal{E} x\right\}_{\nu \in \mathbb{Z}}$ of $\mathcal{V}\left(e_{-1}=\delta(x)\right)$. The action of $x, \partial_{x}, x^{\ell-1} \partial_{x}^{n+\ell-1}$ on $\mathcal{V}$ are written in matrix form with respect to this basis as follows:

$$
\begin{aligned}
x & \leftrightarrow\left((\nu+1) \delta_{\mu-1, \nu}\right)_{\mu, \nu \in \mathbb{Z}}, \\
\partial_{x} & \leftrightarrow\left(\delta_{\mu+1, \nu}\right)_{\mu, \nu \in \mathbb{Z},} \\
x^{\ell-1} \partial_{x}^{n+\ell-1} & \leftrightarrow\left((\nu-n)(\nu-n-1) \cdots(\nu-n-\ell+2) \delta_{\mu+n, \nu}\right)_{\mu, \nu \in \mathbb{Z} .}
\end{aligned}
$$

A decreasing filtaration $\mathcal{V}^{(n)}$ of $\mathcal{V}$ is defined by

$$
\mathcal{V}^{(n)}=\mathcal{E}^{-n} e_{0}=\left\{\xi=\sum_{k=n}^{\infty} \xi_{k} e_{k}, \quad \xi_{k} \in \mathbb{C}\right\}
$$

There is a direct sum decomposition $\mathcal{V}=V^{\phi} \oplus \mathcal{V}^{(0)}$. Based on this decomposition, the universal Grassmann manifold $U G M$ is defined as the set of subspaces of $\mathcal{V}$ which has "the same size" as $V^{\phi}$. Exactly speaking: define the induced filtration of a subspace $U$ of $\mathcal{V}$ by $U^{(k)}:=U \cap \mathcal{V}^{(k)}$. A subspace $U$ lies in $U G M$ if and only if $\operatorname{dim} U^{(-m)}=m$ for $m=0,1,2, \ldots$ (This is the definition of the generic cell of $U G M, U G M^{\phi}$, in the notation of [14]. For our purpose this definition is enough.) The following one-to-one correspondence is fundamental ([14] Theorem 8.1, 9.2):

$$
\mathcal{W} \ni W \mapsto \gamma(W)=W^{-1} V^{\phi} \in U G M
$$

where

$$
\begin{aligned}
\mathcal{W} & =\{\text { monic } 0 \text {-th order micro-differential operators }\} \\
& =\left\{W=1+\sum_{n=1}^{\infty} w_{n}(x) \partial^{-n}\right\}
\end{aligned}
$$


We call the basis $\left\{\xi_{k}=W^{-1} e_{k}\right\}_{k \in \mathbb{N}^{C}}\left(\mathbb{N}^{C}=\{-1,-2, \ldots\}\right)$ of $U$ the canonical frame. Like the finite dimensional Grassmann manifold, the universal Grassmann manifold has the Plücker embedding into the infinite dimensional projective space $\mathbb{P}^{\infty}(\mathbb{C})$ :

$$
U G M \ni U=\bigoplus_{k \in \mathbb{N}^{C}} \mathbb{C} \xi_{k} \mapsto \xi_{-1} \wedge \xi_{-2} \wedge \ldots \in \bigwedge_{k \in \mathbb{N}^{C}} \mathcal{V} \backslash\{0\} \rightarrow \mathbb{P}\left(\bigwedge_{k \in \mathbb{N}^{C}} \mathcal{V}\right)
$$

The image of this embedding, which is naturally identified with $U G M$, is also described as the $G L(\infty)$-orbit of $e_{-1} \wedge e_{-2} \wedge \ldots$ (for details we refer to [15], [28]). The action of the Lie algebra $\mathfrak{g l}(\infty)$ of $G L(\infty)$ on the space of semi-infinite exterior products is neatly written down in terms of the Date-Jimbo-Kashiwara-Miwa (DJKM, for short) free fermions. Here as in [15] we mean by $\mathfrak{g l}(\infty)$ the central extension of the Lie algebra of $\mathbb{Z} \times \mathbb{Z}$ matrices $\overline{\mathfrak{g l}(\infty)}$. The DJKM free fermion operators $\psi_{n}, \psi_{n}^{*}$ act on semi-infinite wedge products as:

$$
\begin{aligned}
\psi_{n}|v\rangle & =e_{n} \wedge|v\rangle, & \langle v| \psi_{n} & =\langle v| \frac{\overleftarrow{\partial}}{\partial e_{n}} \\
\psi_{n}^{*}|v\rangle & =\frac{\vec{\partial}}{\partial e_{n}}|v\rangle, & \langle v| \psi_{n}^{*} & =\langle v| \wedge e_{n}
\end{aligned}
$$

Here $\langle v|$ belongs to $\left(\bigwedge_{\mathbb{N} C} \mathcal{V}\right)^{\vee}$, the dual of $\bigwedge_{\mathbb{N} C} \mathcal{V}$, and is of the form $\ldots \wedge f_{2} \wedge f_{1} \wedge f_{0}$. Notations $\langle v| \overleftarrow{\partial} / \partial e_{n}$ and $\vec{\partial} / \partial e_{n}|v\rangle$ mean eliminating the right- and left-most $e_{n}$ of $\langle v|$ and $|v\rangle$.

These fermion operators satisfy the anti-commutation relations

$$
\left[\psi_{i}, \psi_{j}\right]_{+}=\left[\psi_{i}^{*}, \psi_{j}^{*}\right]_{+}=0, \quad\left[\psi_{i}, \psi_{j}^{*}\right]_{+}=\delta_{i j}
$$

We can identify the Lie algebra $\mathfrak{g l}(\infty)$ with a subalgebra of the Clifford algebra generated by $\psi_{n}, \psi_{m}^{*}$ as follows:

$$
\mathfrak{g l}(\infty) \cong \bigoplus_{n, m \in \mathbb{Z}} \mathbb{C}: \psi_{n} \psi_{m}^{*}: \oplus \mathbb{C} \hat{c}
$$

where : : is the normal ordering defined by : $\psi_{n} \psi_{m}^{*}:=\psi_{n} \psi_{m}^{*}-\left\langle 0\left|\psi_{n} \psi_{m}^{*}\right| 0\right\rangle$, and $\hat{c}$ is a central element. 
The vacuum vector (resp. dual vacuum vector) of charge $s,|s\rangle=e_{s-1} \wedge e_{s-2} \wedge \ldots$ (resp. $\left.\langle s|=\ldots e_{s+1} \wedge e_{s}\right)$, is characterized by

$$
\begin{array}{ll}
\psi_{n}|s\rangle=0 & (n \leqq s-1), \quad \psi_{n}^{*}|s\rangle=0 \quad(n \geqq s), \\
\langle s| \psi_{n}=0 \quad(n \geqq s), \quad\langle s| \psi_{n}^{*}=0 \quad(n \leqq s-1) .
\end{array}
$$

Normalizing the pairing between $(\Lambda \mathcal{V})^{\vee}$ and $\Lambda \mathcal{V}$ by $\left\langle s \mid s^{\prime}\right\rangle=\delta_{s, s^{\prime}}$, we can express the tau function, in general, as a vacuum expectation value,

$$
\tau(t)=\left\langle 0\left|e^{H(t)}\right| U\right\rangle
$$

where $|U\rangle$ is the vector in $\Lambda \mathcal{V}$ corresponding to a point $U$ in the universal Grassmann manifold through (1.7.37). The generator $H(t)$ of the time evolutions is given by

$$
H(t)=\sum_{n=1}^{\infty} t_{n} H_{n}, \quad H_{n}=\sum_{i=-\infty}^{\infty}: \psi_{i} \psi_{i+n}^{*}: .
$$

The Lie algebra $\mathfrak{g l}(\infty)$ acts on the tau function through $(1.7 .38,39,41,43)$ as

$$
\tau(t) \mapsto\left\langle 0\left|e^{H(t)}: \psi_{n} \psi_{m}^{*}:\right| U\right\rangle .
$$

In the presence of the Planck constant $\hbar$ the constant ring of $\mathcal{E}$ and $\mathcal{D}$ becomes $\mathbb{C}((\hbar))$, the ring of rational functions of $\hbar$ around $\hbar=0$, and the basis $\left\{e_{\nu}\right\}$ of $\mathcal{V}$ should be rescaled to $e_{\nu}=(\hbar \partial)^{-1-\nu} \bmod \mathcal{E} x$. Therefore the matrix expression (1.7.34) should be also rescaled to

$$
\begin{aligned}
x & \leftrightarrow\left((\nu+1) \hbar \delta_{\mu-1, \nu}\right)_{\mu, \nu \in \mathbb{Z}}, \\
\hbar \partial & \leftrightarrow\left(\delta_{\mu+1, \nu}\right)_{\mu, \nu \in \mathbb{Z},} \\
x^{\ell-1}(\hbar \partial)^{n+\ell-1} & \leftrightarrow\left((\nu-n)(\nu-n-1) \cdots(\nu-n-\ell+2) \hbar^{\ell-1} \delta_{\mu+n, \nu}\right) .
\end{aligned}
$$

Note that not all points of the universal Grassmann manifold come into the game, since the dressing operators under consideration are restricted to the form (1.7.5).

Proposition 1.7.15. Any tau function with good quasi-classical behaviour can be expressed as:

$$
\tau(\hbar, t)=\left\langle 0\left|e^{H(t) / \hbar} g(\hbar)\right| 0\right\rangle,
$$


where $g(\hbar)$ is the Clifford operator of the form

$$
\begin{gathered}
g(\hbar)=\exp \left(\hbar^{-1} \mathcal{O}_{A}(\hbar)\right), \\
\mathcal{O}_{A}(\hbar)=\oint: A\left(\lambda, \hbar \frac{\partial}{\partial \lambda}\right) \psi(\lambda) \cdot \psi^{*}(\lambda): \frac{d \lambda}{2 \pi i},
\end{gathered}
$$

where

$$
\psi(\lambda)=\sum_{n=-\infty}^{\infty} \psi_{n} \lambda^{n}, \quad \psi^{*}(\lambda)=\sum_{n=-\infty}^{\infty} \psi_{n}^{*} \lambda^{-n-1}
$$

are the free fermion fields introduced in [15] and $A \psi(\lambda) \cdot \psi^{*}(\lambda)$ is understood to be “A $\psi(\lambda)$ times $\psi^{*}(\lambda) "$.

Proof. Since the dressing operator has the form (1.7.5) and is connected to a vector in $\bigwedge \mathcal{V}$ by $(1.7 .36,37)$, we have only to write down the action of $X(\hbar, x, \hbar \partial ; t=0)$ on $\bigwedge \mathcal{V}$ in the fermionic language. This is done by expanding $X(\hbar, x, \hbar \partial ; t=0)$ as

$$
X(\hbar, x, \hbar \partial ; t=0)=\sum_{\substack{\ell \geq 1 \\ n+\ell<1}} a_{n, \ell}(\hbar) x^{\ell-1}(\hbar \partial)^{n+\ell-1},
$$

and replacing $x^{\ell-1}(\hbar \partial)^{n+\ell-1}$ with an element of $\mathfrak{g l}(\infty)$ according to (1.7.46). (The matrix unit $E_{m, n}=\left(\delta_{m, \mu} \delta_{n, \nu}\right)_{\mu, \nu}$ is naturally identified with : $\left.\psi_{m} \psi_{n}^{*}:.\right)$ It is easy to see that $\mathcal{O}_{A}(\hbar)$ of $(1.7 .48)$ with

$$
A(\lambda, \hbar \partial / \partial \lambda)=-\sum_{\substack{\ell \geq 1 \\ n+\ell<1}} a_{n, \ell}(\hbar) \lambda^{n+\ell-1}\left(\hbar \frac{\partial}{\partial \lambda}\right)^{\ell-1}
$$

is a desired expression. Note that the central extension does not affect the result, since the matrix representation of $X$ is lower triangular.

$W_{1+\infty}$ symmetries (1.7.29) are implemented by inserting a fermion bilinear form in $(1.7 .47)$ as

$$
Z(\hbar, \tilde{\lambda}, \lambda) \tau(\hbar, t)=\left\langle 0\left|e^{H(t) / \hbar}: \psi(\tilde{\lambda}) \psi^{*}(\lambda): g(\hbar)\right| 0\right\rangle
$$

This fact was pointed out by Date et al. in the case of $\hbar=1$. In particular, insertion of

$$
\mathcal{O}_{k}^{(\ell)}(\hbar)=\oint: \lambda^{n+\ell-1}\left(\hbar \frac{\partial}{\partial \lambda}\right)^{\ell-1} \psi(\lambda) \cdot \psi^{*}(\lambda): \frac{d \lambda}{2 \pi i},
$$

where the integral is along a circle $|\lambda|=$ const., corresponds to the action of $\hbar^{-1} W_{n}^{(\ell)}(\hbar)$ on $\tau(\hbar, t)$ defined through $(1.7 .30)$. 
Proposition 1.7.16. The symmetry of the tau function by $W_{n}^{(\ell)}(\hbar)(1.7 .30,53)$ induces a symmetry of $L, M, W$ of the form (1.7.27) with $Y(\hbar, x, \hbar \partial)=-x^{\ell-1}(\hbar \partial)^{n+\ell-1}$

Proof. Denote by $U$ and $W$ the point in $U G M$ and the dressing operator under consideration respectively. The fermion bilinear form $\mathcal{O}_{k}^{(\ell)}$ corresponds to $-Y=x^{\ell-1}(\hbar \partial)^{n+\ell-1}$ through (1.7.46), when one neglects the central extension. Since the central term changes the tau function only by constant multiplication, it does not change $L, M$, $W$. Hence the point

$$
\exp \left(\varepsilon \mathcal{O}_{k}^{(\ell)}(\hbar)\right)|U\rangle=\exp (-\varepsilon Y) W^{-1}(t=0) V^{\phi}
$$

in the universal Grassmann manifold corresponds to $W_{\varepsilon}(t=0)$ of (1.7.28) by the oneto-one mapping (1.7.36). This proves the proposition.

This explains the coincidence (1.7.32) of the quasi-classical limit of $W_{1+\infty}$ symmetries (1.7.31) with the symmetries defined through the deformation of the twistor data §1.6.

\subsection{Special solutions.}

Many special solutions to the dispersionless KP hierarchy are constructed, e.g., by [6], but here we concentrate our attention to those solutions which appear in the context of topological minimal models [29], [30], [8]. In fact, Krichever's work on this subject [7] was the starting point of our study [1] where we gave an interpretation of his results in our framework. Dubrovin [8] studied these solutions from the viewpoint of integrable systems of hydrodynamic type [31].

Krichever showed in [7] that the perturbed chiral rings of the topological minimal models (Landau-Ginzurg models of $A_{N}$-type) can be described by some special solutions of the dispersionless KP hierarchy ("dispersionless Lax equations" in his terminology). These solutions are characterized by the twistor data:

$$
f(k, x)=k^{N} / N, \quad g(k, x)=x k^{1-N} .
$$

In other words, those solutions are determined by the equations:

$$
(\mathcal{P})_{\leq-1}=0, \quad(\mathcal{Q})_{35} \leq-1=0
$$


where $\mathcal{P} \underset{\text { def }}{=} \mathcal{L}^{N} / N, \mathcal{Q} \underset{\text { def }}{=} \mathcal{M L}^{1-N}$. Using the theory of the generalized hodograph transformation by Tsarev [32], we can show the solvability of this Riemann-Hilbert decomposition problem.

Proposition 1.8.1. ([1] Proposition 8, 9, 10) The equations (1.8.2) has a solution $(\mathcal{L}, \mathcal{M})$ such that the coefficeitns $u_{n}$ of $\mathcal{L}$ (1.1.2) and $v_{i}$ of $\mathcal{M}$ (1.3.2) are homogeneous of degree zero and one with respect to the degree defined by $\operatorname{deg}\left(t_{n}\right)=1$. This solution is defined in a domain where $t_{N+1} \neq 0$ and $t_{n} / t_{N+1}(n \neq N+1)$ are sufficiently small. The tau function, up to an integration constant, is given by

$$
\log \tau_{\mathrm{dKP}}=\frac{1}{2} \sum_{n=1}^{\infty} t_{n} v_{n}+\text { const. }
$$

or, equivalently, by

$$
\log \tau_{\mathrm{dKP}}=\frac{1}{2} \sum_{n, m=1}^{\infty} t_{m} t_{n} \frac{\partial v_{n}}{\partial t_{m}}+\text { const. }
$$

As Krichever pointed out in [7], this tau function restricted to the "small phase space" $\left\{t_{N+1}=1 /(N+1), t_{N+2}=t_{N+3}=\ldots=0\right\}$ gives the free energy of the Landau-Ginzburg model of $A_{N-1}$-type. Furthermore, according to recent developments in the topological field theory [33], the topological matter fields coupled to topological gravity is described by this solution of the dispersionless KP hierarchy in the "full phase space", where the higher times $t_{n}(n \geqq N+2)$ play the role of gravitational coupling constants.

Krichever's dispersionless analogue of Virasoro constraints is interpreted as the following "dispersionless $w_{1+\infty}$ constraints", which is a direct consequence of the results in $\S 1.6$ and derived from the basic relations

$$
\left(\mathcal{L}^{(m+1) N+n(1-N)} \mathcal{M}^{n}\right)_{\leq-1}=\text { const. }\left(\mathcal{P}^{m+1} \mathcal{Q}^{n}\right)_{\leq-1}=0
$$

for $m \geqq-1, n \geqq 0$.

Proposition 1.8.2. ([1] Proposition 11, 17; [7]) The tau function $\tau=\tau_{0}(t)$ of Proposition 1.8 .1 satisfies the constraints

$$
\delta_{\lambda^{(m+1) N+n(1-N)} x^{n}} \log \tau=-\frac{1}{n+1} \operatorname{Res}\left[\mathcal{L}^{(m+1) N+n(1-N)} \mathcal{M}^{n+1} d_{\lambda} \mathcal{L}\right]=0
$$


for $m \geqq-1$ and $n \geqq 0$. In particular, the constraints for $(m, n)=(-1,1),(0,0)$ and $m=1,2, \ldots, n=1$ give the Krichever's dispersionless Virasoro constraitns:

$$
\begin{aligned}
& \sum_{k=N+1}^{\infty} k t_{k} \frac{\partial \log \tau}{\partial t_{k-N}}+\frac{1}{2} \sum_{i=1}^{N-1} i(N-i) t_{i} t_{N-i}=0, \\
& \sum_{k=1}^{\infty} k t_{k} \frac{\partial \log \tau}{\partial t_{k}}=0, \\
& \sum_{k=1}^{\infty} k t_{k} \frac{\partial \log \tau}{\partial t_{k+m N}}+\frac{1}{2} \sum_{i=1}^{m N-1} \frac{\partial \log \tau}{\partial t_{i}} \frac{\partial \log \tau}{\partial t_{m N-i}}=0 .
\end{aligned}
$$

We can deform this solution by changing the twistor data (1.8.1) to

$$
f(k, x)=k^{N} / N, \quad g(k, x)=x k^{1-N}+h(k),
$$

where $h(k)=\sum_{n \in \mathbb{Z}} h_{n} k^{n}$ is an arbitrary Laurent series in $k$ (for the sake of simplicity, let us suppose that $\left.h_{-N}=0\right)$. The corresponding $(\mathcal{P}, \mathcal{Q})$-pair is now given by

$$
\mathcal{P}=\mathcal{L}^{N} / N, \quad \mathcal{Q}=\mathcal{L}^{1-N} \mathcal{M}+h(\mathcal{L})
$$

Proposition 1.8.3. ([1] Proposition 12.) Let $\tau_{0}=\tau_{0}(t)$ be the tau function of the undeformed solution $(h=0)$. The tau function $\tau_{h}=\tau_{h}(t)$ of the deformed solution is then given by

$$
\begin{aligned}
\tau_{h}(t)= & \text { const. } \exp \left(-\sum_{n=1}^{\infty} h_{-n-N} t_{n}\right) \\
& \times \tau_{0}\left(t_{1}+h_{1-N}, t_{2}+\frac{h_{2-N}}{2}, \ldots, t_{k}+\frac{h_{k-N}}{k}, \ldots\right) .
\end{aligned}
$$

The above solution of the dispersionless KP hierarchy has a natural "dispersionful" extension in the context of topological string theory. Namely, the (generalized) Kontsevich models, a version of the topological string theory intoduced by [34], [35], specify a solution of the ordinary KP hierarchy whose quasi-classical limit gives solutions of the dispersionless KP hierarchy of the above type. Actually the "quasi-classical" limit is 
the contribution from the genus $g=0$ Riemann surfaces in the context of the string theory. The corresponding Riemann-Hilbert problem in the sense of $\S 1.7 .5$ is

$$
\frac{1}{N}\left(L^{N}\right)_{\leq-1}=0, \quad\left(M L^{1-N}+\frac{N-1}{2} \hbar L^{-N}+N L\right)_{\leq-1}=0
$$

which has quasi-classical limit of the form (1.8.10), or (1.8.1) up to a suitable shift of time variables. This model is subject to the Virasoro constraints [30], [36], [35], [37] and the quasi-classical limit of this constraints are the constraints stated in Proposition 1.8.2. For details on the quasi-classical limit of the generalized Kontsevich model we refer to [38].

\section{Dispersionless Toda hierarchy}

\subsection{Lax formalism.}

Here we briefly review the definition of the dispersionless Toda hierarchy, mostly following $[2,4]$. In contrast to the (dispersionless) KP hierarchy the dispersionless Toda hierarchy has two series of independent ("time") variables $t=\left(t_{1}, t_{2}, \ldots\right)$ and $\bar{t}=\left(\bar{t}_{1}, \bar{t}_{2}, \ldots\right)$ along with a new spatial variable $s$. Its Lax representation is:

$$
\begin{aligned}
\frac{\partial \mathcal{L}}{\partial t_{n}}=\left\{\mathcal{B}_{n}, \mathcal{L}\right\}, & \frac{\partial \mathcal{L}}{\partial \bar{t}_{n}}=\left\{\overline{\mathcal{B}}_{n}, \mathcal{L}\right\}, \\
\frac{\partial \overline{\mathcal{L}}}{\partial t_{n}}=\left\{\mathcal{B}_{n}, \overline{\mathcal{L}}\right\}, & \frac{\partial \overline{\mathcal{L}}}{\partial \bar{t}_{n}}=\left\{\overline{\mathcal{B}}_{n}, \overline{\mathcal{L}}\right\}, \quad n=1,2, \ldots,
\end{aligned}
$$

where $\mathcal{L}$ and $\overline{\mathcal{L}}$ are Laurent series

$$
\begin{aligned}
\mathcal{L} & =p+\sum_{n=0}^{\infty} u_{n+1}(t, \bar{t}, s) p^{-n} \\
\overline{\mathcal{L}}^{-1} & =\bar{u}_{0}(t, \bar{t}, s) p^{-1}+\sum_{n=0}^{\infty} \bar{u}_{n+1}(t, \bar{t}, s) p^{n},
\end{aligned}
$$

of a variable $p$, and $\mathcal{B}_{n}$ and $\overline{\mathcal{B}}_{n}$ are given by

$$
\mathcal{B}_{n}=\left(\mathcal{L}^{n}\right)_{\geq 0}, \quad \overline{\mathcal{B}}_{n}=\left(\overline{\mathcal{L}}^{-n}\right)_{\leq-1}
$$

Note that we now define the coefficients $\bar{u}_{n+1}$ to be those of the expansion of $\overline{\mathcal{L}}^{-1}$ rather than $\overline{\mathcal{L}}$ itself Here ()$_{\geq 0}$ and ()$_{\leq-1}$ denote the projection of Laurent series 
onto a linear combination of $p^{n}$ with $n \geqq 0$ and $\leqq-1$ respectively. The Poisson bracket $\{$,$\} is defined by$

$$
\{A(p, s), B(p, s)\}=p \frac{\partial A(p, s)}{\partial p} \frac{\partial B(p, s)}{\partial s}-p \frac{\partial A(p, s)}{\partial s} \frac{\partial B(p, s)}{\partial p} .
$$

By this definition, $\log p$ and $s$ are cononically conjugate coordinates on the two dimensional "phase space".

By the same argument as in [21], one can easily prove the following fact.

Proposition 2.1.1. The Lax equations for $\mathcal{L}$ are equivalent to the "zero-curvature equations"

$$
\begin{aligned}
& \partial_{t_{n}} \mathcal{B}_{m}-\partial_{t_{m}} \mathcal{B}_{n}+\left\{\mathcal{B}_{m}, \mathcal{B}_{n}\right\}=0 \\
& \partial_{\bar{t}_{n}} \overline{\mathcal{B}}_{m}-\partial_{\bar{t}_{m}} \overline{\mathcal{B}}_{n}+\left\{\overline{\mathcal{B}}_{m}, \overline{\mathcal{B}}_{n}\right\}=0 \\
& \partial_{\bar{t}_{n}} \mathcal{B}_{m}-\partial_{t_{m}} \overline{\mathcal{B}}_{n}+\left\{\mathcal{B}_{m}, \overline{\mathcal{B}}_{n}\right\}=0
\end{aligned}
$$

In our ealier work $[2,4]$ we formulated the hierarchy assuming the existence of $\mathcal{M}$ and $\overline{\mathcal{M}}$ from the very beginning. We now start from the above formulation and show in $\S 2.3$ how $\mathcal{M}$ and $\overline{\mathcal{M}}$ enter into the story.

The dispersionless (or long-wave) limit $\phi$ of the original Toda field is hidden in this formalism in the following way.

Proposition 2.1.2. There exists $\phi=\phi(t, \bar{t}, s)$ characterized by

$$
d \phi=\sum_{n=1}^{\infty} \operatorname{Res}\left(\mathcal{L}^{n} d \log p\right) d t_{n}-\sum_{n=1}^{\infty} \operatorname{Res}\left(\overline{\mathcal{L}}^{-n} d \log p\right) d \bar{t}_{n}+\log \bar{u}_{0} d s
$$

where " $d "$ means total differentiation in $(t, \bar{t}, s)$, and $d \log p=d p / p$. Furthermore $\phi$ satisfies the well-known dispersionless (long-wave) limit of the two-dimensional Toda field equation

$$
\frac{\partial^{2} \phi}{\partial t_{1} \partial \bar{t}_{1}}+\frac{\partial}{\partial s} \exp \left(\frac{\partial \phi}{\partial s}\right)=0
$$

Proof. The equation (2.1.6) is a compact form of the following system.

$$
\begin{aligned}
\partial_{t_{n}} \phi & =b_{n, 0} \underset{\text { def }}{=}\left(\mathcal{L}^{n}\right)_{0}, \\
\partial_{\bar{t}_{n}} \phi & =-\bar{b}_{n, 0} \underset{\text { def }}{=}-\left(\overline{\mathcal{L}}^{-n}\right)_{0}, \\
\partial_{s} \phi & =\log \bar{u}_{0},
\end{aligned}
$$


where $(\quad)_{0} \underset{\text { def }}{=} \operatorname{Res}(\quad) d \log p$ is the projection to the constant term with respect to $p$. The compatibility conditions among these equations are contained in the Lax equations (2.1.1) and the zero-curvature equations (2.1.5). For example, the compatibility of $(2.1 .8)_{n}$ and $(2.1 .8)_{m}, \partial_{t_{n}} b_{m, 0}=\partial_{t_{m}} b_{n, 0}$, can be read from the coefficient of $p^{0}$ of the first equation of (2.1.5). The compatibility conditions between $(2.1 .8-9)_{n}$ and $(2.1 .10)$ $\partial_{s} b_{n, 0}=\partial_{t_{n}} \log \bar{u}_{0}, \partial_{s} \bar{b}_{n, 0}=-\partial_{\bar{t}_{n}} \log \bar{u}_{0}$ come from the $p^{1}$ part of the Lax equations (2.1.1) for $\overline{\mathcal{L}}$. The others can be verified similarly.

The equation (2.1.7) is a direct consequence of $(2.1 .8-10)$ and one of $(2.1 .5), \partial_{\bar{t}_{1}} \mathcal{B}_{1}-$ $\partial_{t_{1}} \overline{\mathcal{B}}_{1}+\left\{\mathcal{B}_{1}, \overline{\mathcal{B}}_{1}\right\}=0$

Like the ordinary Toda lattice hierarchy [39], we can exchange the roles of $\mathcal{L}$ and $\overline{\mathcal{L}}$ by a "gauge-transformation".

Lemma 2.1.3. Define $\mathcal{L}^{\prime} \underset{\text { def }}{=} \exp (-\operatorname{ad} \phi) \mathcal{L}, \overline{\mathcal{L}}^{\prime} \underset{\text { def }}{=} \exp (-\operatorname{ad} \phi) \overline{\mathcal{L}}$. Then they are of the form

$$
\begin{aligned}
\mathcal{L}^{\prime} & =\bar{u}_{0}^{-1}(t, \bar{t}, s) p+\sum_{n=0}^{\infty} u_{n+1}^{\prime}(t, \bar{t}, s) p^{-n}, \\
\left(\overline{\mathcal{L}}^{\prime}\right)^{-1} & =p^{-1}+\sum_{n=0}^{\infty} \bar{u}_{n+1}^{\prime}(t, \bar{t}, s) p^{n}
\end{aligned}
$$

and satisfy the Lax equations

$$
\begin{array}{ll}
\frac{\partial \mathcal{L}^{\prime}}{\partial t_{n}}=\left\{\mathcal{B}_{n}^{\prime}, \mathcal{L}^{\prime}\right\}, & \frac{\partial \mathcal{L}^{\prime}}{\partial \bar{t}_{n}}=\left\{\overline{\mathcal{B}}_{n}^{\prime}, \mathcal{L}^{\prime}\right\}, \\
\frac{\partial \overline{\mathcal{L}}^{\prime}}{\partial t_{n}}=\left\{\mathcal{B}_{n}^{\prime}, \overline{\mathcal{L}}^{\prime}\right\}, & \frac{\partial \overline{\mathcal{L}}^{\prime}}{\partial \bar{t}_{n}}=\left\{\overline{\mathcal{B}}_{n}^{\prime}, \overline{\mathcal{L}}^{\prime}\right\}, \quad n=1,2, \ldots,
\end{array}
$$

as well as the zero-curvature equations

$$
\begin{aligned}
& \partial_{t_{n}} \mathcal{B}_{m}^{\prime}-\partial_{t_{m}} \mathcal{B}_{n}^{\prime}+\left\{\mathcal{B}_{m}^{\prime}, \mathcal{B}_{n}^{\prime}\right\}=0, \\
& \partial_{\bar{t}_{n}} \overline{\mathcal{B}}_{m}^{\prime}-\partial_{\bar{t}_{m}} \overline{\mathcal{B}}_{n}^{\prime}+\left\{\overline{\mathcal{B}}_{m}^{\prime}, \overline{\mathcal{B}}_{n}^{\prime}\right\}=0, \\
& \partial_{\bar{t}_{n}} \mathcal{B}_{m}^{\prime}-\partial_{t_{m}} \overline{\mathcal{B}}_{n}^{\prime}+\left\{\mathcal{B}_{m}^{\prime}, \overline{\mathcal{B}}_{n}^{\prime}\right\}=0 .
\end{aligned}
$$

Here $\mathcal{B}_{n}^{\prime} \underset{\text { def }}{=}\left(\left(\mathcal{L}^{\prime}\right)^{n}\right)_{\geq 1}$ and $\overline{\mathcal{B}}_{n}^{\prime} \underset{\text { def }}{=}\left(\left(\overline{\mathcal{L}}^{\prime}\right)^{-n}\right)_{\leq 0}$.

Proof. It is easy to prove $(2.1 .11)$ by $(2.1 .10)$. The equations $(2.1 .11,12)$ are results of Lemma A.1 and (2.1.8-9). 
Remark 2.1.4. There is a one-parameter family of gauge as in [39]. The above one will be used in the next section.

\subsection{Dressing operators.}

Here like in $\S 1.2$, we introduce the dressing functions $\varphi$ and $\bar{\varphi}$ in a Lie-algebraic sense.

Proposition 2.2.1. i) Let $\mathcal{L}$ and $\overline{\mathcal{L}}$ be a solution of the dispersionless Toda hierarchy (2.1.1). Then there exists a pair of Laurent series $\varphi$ and $\bar{\varphi}$ of $p$ such that

$$
\begin{array}{ll}
\mathcal{L}=e^{\operatorname{ad} \varphi}(p), & \overline{\mathcal{L}}=e^{\operatorname{ad} \phi} e^{\operatorname{ad} \bar{\varphi}}(p), \\
\nabla_{t_{n}, \varphi} \varphi=-\left(\mathcal{L}^{n}\right)_{\leq-1}, & e^{\operatorname{ad} \phi} \nabla_{t_{n}, \bar{\varphi}} \bar{\varphi}+\partial_{t_{n}} \phi=\left(\mathcal{L}^{n}\right)_{\geq 0}, \\
\nabla_{\bar{t}_{n}, \varphi} \varphi=\left(\overline{\mathcal{L}}^{-n}\right)_{\leq-1}, & e^{\operatorname{ad} \phi} \nabla_{\bar{t}_{n}, \bar{\varphi}} \bar{\varphi}+\partial_{\bar{t}_{n}} \phi=-\left(\overline{\mathcal{L}}^{-n}\right)_{\geq 0}
\end{array}
$$

for $n=1,2, \ldots$, where $\phi$ is the dispersionless limit of the Toda field introduced in Lemma 2.1.2, $\varphi$ and $\bar{\varphi}$ are Laurent series of the form $\varphi(t, \bar{t}, p, s)=\sum_{n=1}^{\infty} \varphi_{n}(t, \bar{t}, s) p^{-n}$, $\bar{\varphi}(t, \bar{t}, p, s)=\sum_{n=1}^{\infty} \bar{\varphi}_{n}(t, \bar{t}, s) p^{n}$, and ad and $\nabla$ have the same meaning as in $\S 1.2$.

Such $\varphi, \bar{\varphi}$ are determined uniquely up to transformations $\varphi \mapsto H(\varphi, \psi), \bar{\varphi} \mapsto$ $H(\bar{\varphi}, \bar{\psi})$, where $H(X, Y)$ is the Hausdorff series (cf. Appendix A)

$$
e^{\operatorname{ad} H(\varphi, \psi)}=e^{\operatorname{ad} \varphi} e^{\operatorname{ad} \psi}, \quad e^{\operatorname{ad} H(\bar{\varphi}, \bar{\psi})}=e^{\operatorname{ad} \bar{\varphi}} e^{\operatorname{ad} \bar{\psi}},
$$

and $\psi$ and $\bar{\psi}$ are Laurent series of the form $\psi=\sum_{n=1}^{\infty} \psi_{n} p^{-n}, \bar{\psi}=\sum_{n=1}^{\infty} \bar{\psi}_{n} p^{n}\left(\psi_{n}\right.$, $\bar{\psi}_{n}:$ constant).

ii) Conversely, if $\varphi(t, \bar{t}, p, s)=\sum_{n=1}^{\infty} \varphi_{n}(t, \bar{t}, s) p^{-n}$ and $\bar{\varphi}(t, \bar{t}, p, s)=\sum_{n=1}^{\infty} \bar{\varphi}_{n}(t, \bar{t}, s) p^{n}$ satisfy (2.2.2) and (2.2.3), then $\mathcal{L}$ and $\overline{\mathcal{L}}$ defined in (2.2.1) give a solution of the dispersionless Toda hierarchy (2.1.1).

Proof traces that of Proposition 1.2.1. Let $\varphi^{0}$ be an arbitrary series of the form $\varphi^{0}(t, \bar{t}, p, s)=\sum_{n=1}^{\infty} \varphi_{n}^{0}(t, \bar{t}, s) p^{-n}$ such that $\mathcal{L}=\exp \left(\operatorname{ad} \varphi^{0}\right)$. Then the zero-curvature equations (2.1.5) imply the integrability of the system of equations

$$
\frac{\partial \varphi^{\prime}}{\partial t_{n}}=p^{n}-\mathcal{B}_{n}^{\varphi^{0}}, \quad \frac{\partial \varphi^{\prime}}{\partial \bar{t}_{n}}=\overline{\mathcal{B}}_{n}^{\varphi^{0}}
$$


for $\varphi^{\prime}(t, \bar{t}, p, s)=\sum_{n=1}^{\infty} \varphi_{n}^{\prime}(t, \bar{t}, s) p^{-n}$, where

$$
\mathcal{B}_{n}^{\varphi^{0}} \underset{\text { def }}{=} e^{-\operatorname{ad} \varphi^{0}}\left(\mathcal{B}_{n}-\nabla_{t_{n}, \varphi^{0}} \varphi^{0}\right), \quad \overline{\mathcal{B}}_{n}^{\varphi^{0}} \underset{\text { def }}{=} e^{-\operatorname{ad} \varphi^{0}}\left(\overline{\mathcal{B}}_{n}-\nabla_{\bar{t}_{n}, \varphi^{0}} \varphi^{0}\right)
$$

Due to the Lax equations (2.1.1) $\mathcal{B}_{n}^{\varphi^{0}}$ and $\overline{\mathcal{B}}_{n}^{\varphi^{0}}$ are independent of $s$. Hence there is a solution of (2.2.4) which is also independent of $s$. Using the Hausdorff-Campbell formula, we define $\exp (\varphi)=\exp \left(\varphi^{\prime}\right) \exp \left(\varphi^{0}\right)$, and this $\varphi$ is a desired dressing function.

We can easily prove the corresponding statements for $\bar{\varphi}$, using Lemma 2.1.3.

\subsection{Orlov function and Darboux coordinates.}

The Orlov functions $\mathcal{M}$ and $\overline{\mathcal{M}}$ are defined for the dispersionless Toda hierarchy by

$$
\begin{aligned}
& \mathcal{M}=e^{\operatorname{ad} \varphi}\left(s+\sum_{n=1}^{\infty} n t_{n} p^{n}\right)=e^{\operatorname{ad} \varphi} e^{\operatorname{ad} t(p)}(s), \\
& \overline{\mathcal{M}}=e^{\operatorname{ad} \phi} e^{\operatorname{ad} \bar{\varphi}}\left(s-\sum_{n=1}^{\infty} n \bar{t}_{n} p^{-n}\right)=e^{\operatorname{ad} \phi} e^{\operatorname{ad} \bar{\varphi}} e^{\operatorname{ad} \bar{t}\left(p^{-1}\right)}(s),
\end{aligned}
$$

where $t(p)=\sum_{n=1}^{\infty} t_{n} p^{n}, \bar{t}\left(p^{-1}\right)=\sum_{n=1}^{\infty} \bar{t}_{n} p^{-n}$. They have an expansion like

$$
\begin{aligned}
& \mathcal{M}=\sum_{n=1}^{\infty} n t_{n} \mathcal{L}^{n}+s+\sum_{n=1}^{\infty} v_{n}(t, \bar{t}, s) \mathcal{L}^{-n} \\
& \overline{\mathcal{M}}=-\sum_{n=1}^{\infty} n \bar{t}_{n} \overline{\mathcal{L}}^{-n}+s+\sum_{n=1}^{\infty} \bar{v}_{n}(t, \bar{t}, s) \overline{\mathcal{L}}^{n} .
\end{aligned}
$$

As in the proof of Proposition 1.3.1, we can prove:

Proposition 2.3.1. i) The series $\mathcal{M}$ and $\overline{\mathcal{M}}$ satisfy the Lax equations

$$
\begin{array}{ll}
\frac{\partial \mathcal{M}}{\partial t_{n}}=\left\{\mathcal{B}_{n}, \mathcal{M}\right\}, & \frac{\partial \mathcal{M}}{\partial \bar{t}_{n}}=\left\{\overline{\mathcal{B}}_{n}, \mathcal{M}\right\}, \\
\frac{\partial \overline{\mathcal{M}}}{\partial t_{n}}=\left\{\mathcal{B}_{n}, \overline{\mathcal{M}}\right\}, & \frac{\partial \overline{\mathcal{M}}}{\partial \bar{t}_{n}}=\left\{\overline{\mathcal{B}}_{n}, \overline{\mathcal{M}}\right\}, \quad n=1,2, \ldots,
\end{array}
$$

and the canonical Poisson relations $\{\mathcal{L}, \mathcal{M}\}=\mathcal{L}$ and $\{\overline{\mathcal{L}}, \overline{\mathcal{M}}\}=\overline{\mathcal{L}}$.

ii) Conversely, if the potential $\phi$ is given, and if a pair of series $\mathcal{M}$ and $\overline{\mathcal{M}}$ of the form (2.3.2) satisfy (2.3.3) and the canonical Poisson relations, then there exist unique dressing functions $\varphi$ and $\bar{\varphi}$ which are connected with $\mathcal{M}$ and $\overline{\mathcal{M}}$ through (2.3.1). 
Explicitly, $\varphi(t, \bar{t}, p, s)=\sum_{n=1}^{\infty} \varphi_{n}(t, \bar{t}, s) p^{-n}$ and $\bar{\varphi}(t, \bar{t}, p, s)=\sum_{n=1}^{\infty} \bar{\varphi}_{n}(t, \bar{t}, s) p^{n}$, and the coefficients are of the form

$$
\begin{aligned}
& \varphi_{n}(t, \bar{t}, s)=-\frac{v_{n+1}}{n}+\left(\text { a differential polynomial of } v_{2}, \ldots, v_{n}, u_{2}, \ldots, u_{n-1}\right) . \\
& \bar{\varphi}_{n}(t, \bar{t}, s)=-\frac{\bar{v}_{n+1}}{n}+\left(\text { a differential polynomial of } \phi, \bar{v}_{2}, \ldots, \bar{v}_{n}, \bar{u}_{2}, \ldots, \bar{u}_{n-1}\right) .
\end{aligned}
$$

These pairs of series $(\mathcal{L}, \mathcal{M})$ and $(\overline{\mathcal{L}}, \overline{\mathcal{M}})$ play the role of Darboux coordinates, as is the case with the dispersionless KP hierarchy. The fundamental 2-form is

$$
\omega \underset{\text { def }}{=} \frac{d p}{p} \wedge d s+\sum_{n=1}^{\infty} d \mathcal{B}_{n} \wedge d t_{n}+\sum_{n=1}^{\infty} d \overline{\mathcal{B}}_{n} \wedge d \bar{t}_{n}
$$

which satisfies

$$
d \omega=0, \quad \omega \wedge \omega=0
$$

In fact, (2.3.6) is equivalent to the zero-curvature equations (2.1.5).

Proposition 2.3.2. The dispersionless Toda hierarchy is equivalent to the exterior differential equations

$$
\frac{d \mathcal{L} \wedge d \mathcal{M}}{\mathcal{L}}=\omega=\frac{d \overline{\mathcal{L}} \wedge d \overline{\mathcal{M}}}{\overline{\mathcal{L}}}
$$

Namely, if $(\mathcal{L}, \mathcal{M})$ and $(\overline{\mathcal{L}}, \overline{\mathcal{M}})$ are Laurent series of the form (2.1.2) and (2.3.2) and subject to (2.3.7), then $(\mathcal{L}, \overline{\mathcal{L}})$ give a solution of the dispersionless Toda hierarchy (2.1.1), and $(\mathcal{M}, \overline{\mathcal{M}})$ are corresponding Orlov functions.

Proof. The canonical Poisson relations $\{\mathcal{L}, \mathcal{M}\}=\mathcal{L},\{\overline{\mathcal{L}}, \overline{\mathcal{M}}\}=\overline{\mathcal{L}}$ (resp. the Lax equations $(2.1 .1),(2.3 .3))$ can be easily derived from (2.3.7) by comparing coefficeints of $d p \wedge d s,\left(\right.$ resp. $d p \wedge d t_{n}$ and $d s \wedge d t_{n}$ or $d p \wedge d \bar{t}_{n}$ and $\left.d s \wedge d \bar{t}_{n}\right)$ of both hand sides.

The converse can be proved by tracing back this derivation.

\section{4. $S$ function, $\tau$ function (free energy).}

Here we introduce three potentials $S, \bar{S}$ and $\log \tau_{\text {dToda }}$. Later in $\S 2.7$ we will see that $S$ and $\bar{S}$ are phase funcitons of WKB analysis like the $S$ function for the dispersionless KP hierarchy. 
Proposition 2.4.1. There exist functions $S$ and $\bar{S}$ which satisfy

$$
\begin{aligned}
& d S=\mathcal{M} d \log \mathcal{L}+\log p d s+\sum_{n=1}^{\infty} \mathcal{B}_{n} d t_{n}+\sum_{n=1}^{\infty} \overline{\mathcal{B}}_{n} d \bar{t}_{n} \\
& d \bar{S}=\overline{\mathcal{M}} d \log \overline{\mathcal{L}}+\log p d s+\sum_{n=1}^{\infty} \mathcal{B}_{n} d t_{n}+\sum_{n=1}^{\infty} \overline{\mathcal{B}}_{n} d \bar{t}_{n}
\end{aligned}
$$

where $d \log \mathcal{L}=d \mathcal{L} / \mathcal{L}$. Explicitly, $S$ and $\bar{S}$ have a Laurent expansion of the following form.

$$
\begin{aligned}
& S=\sum_{n=1}^{\infty} t_{n} \mathcal{L}^{n}+s \log \mathcal{L}-\sum_{n=1}^{\infty} \frac{v_{n}}{n} \mathcal{L}^{-n}, \\
& \bar{S}=\sum_{n=1}^{\infty} \bar{t}_{n} \overline{\mathcal{L}}^{-n}+s \log \overline{\mathcal{L}}+\phi+\sum_{n=1}^{\infty} \frac{\bar{v}_{n}}{n} \overline{\mathcal{L}}^{n} .
\end{aligned}
$$

Proof. The existence of $S$ and $\bar{S}$ satisfying (2.4.1) is obvious since the right hand sides of (2.4.1) are closed forms because of (2.3.7). To derive (2.4.2), we have to prove that $S$ and $\bar{S}$ satisfy the following equations.

$$
\begin{aligned}
& \left.\frac{\partial S}{\partial \log \mathcal{L}}\right|_{t, \bar{t}, s \text { fixed }} \quad=\mathcal{M},\left.\quad \frac{\partial \bar{S}}{\partial \log \overline{\mathcal{L}}}\right|_{t, \bar{t}, s \text { fixed }}=\overline{\mathcal{M}} \\
& \left.\frac{\partial S}{\partial t_{n}}\right|_{\mathcal{L}, t_{m}(m \neq n), \bar{t}, s \text { fixed }}=\mathcal{B}_{n},\left.\quad \frac{\partial \bar{S}}{\partial t_{n}}\right|_{\mathcal{L}, t_{m}(m \neq n), \bar{t}, s \text { fixed }}=\mathcal{B}_{n}, \\
& \left.\frac{\partial S}{\partial \bar{t}_{n}}\right|_{\mathcal{L}, t, \bar{t}_{m}(m \neq n), s \text { fixed }}=\overline{\mathcal{B}}_{n},\left.\quad \frac{\partial \bar{S}}{\partial \bar{t}_{n}}\right|_{\mathcal{L}, t, \bar{t}_{m}(m \neq n), s \text { fixed }}=\overline{\mathcal{B}}_{n}, \\
& \left.\frac{\partial S}{\partial s}\right|_{\mathcal{L}, t, \bar{t} \text { fixed }} \quad=\log p,\left.\quad \frac{\partial \bar{S}}{\partial s}\right|_{\mathcal{L}, t, \bar{t} \text { fixed }}=\log p \text {. }
\end{aligned}
$$

Equations (2.4.3) are a direct consequence of the construction (2.4.2). In order to prove the remaining equations $(2.4 .4-6)$, we prepare:

\section{Lemma 2.4.2.}

$$
\begin{aligned}
\frac{\partial v_{m}}{\partial t_{n}} & =\operatorname{Res} \mathcal{L}^{m} d_{p} \mathcal{B}_{n}, & \frac{\partial \bar{v}_{m}}{\partial t_{n}} & =\operatorname{Res} \overline{\mathcal{L}}^{-m} d_{p} \mathcal{B}_{n}, \\
\frac{\partial v_{m}}{\partial \bar{t}_{n}} & =\operatorname{Res} \mathcal{L}^{m} d_{p} \overline{\mathcal{B}}_{n}, & \frac{\partial \bar{v}_{m}}{\partial \bar{t}_{n}} & =\operatorname{Res} \overline{\mathcal{L}}^{-m} d_{p} \overline{\mathcal{B}}_{n}, \\
\frac{\partial v_{m}}{\partial s} & =\operatorname{Res} \mathcal{L}^{m} d \log p, & \frac{\partial \bar{v}_{m}}{\partial s} & =\operatorname{Res} \overline{\mathcal{L}}^{-m} d \log p,
\end{aligned}
$$


Proof. As an example, we prove the first equation of (2.4.7). By the chain rule

$$
\frac{\partial \mathcal{M}}{\partial t_{n}}=\left.\frac{\partial \mathcal{M}}{\partial \mathcal{L}}\right|_{t, v \text { fixed }} \frac{\partial \mathcal{L}}{\partial t_{n}}+n \mathcal{L}^{n}+\sum_{m=1}^{\infty} \frac{\partial v_{m}}{\partial t_{n}} \mathcal{L}^{-m},
$$

which implies

$$
\begin{aligned}
\frac{\partial v_{m}}{\partial t_{n}} & =\operatorname{Res} \mathcal{L}^{m-1}\left(\frac{\partial \mathcal{M}}{\partial t_{n}} d_{p} \mathcal{L}-\left.\frac{\partial \mathcal{M}}{\partial \mathcal{L}}\right|_{t, v \text { fixed }} \frac{\partial \mathcal{L}}{\partial t_{n}} d_{p} \mathcal{L}\right) \\
& =\operatorname{Res} \mathcal{L}^{m-1}\left(\left\{\mathcal{B}_{n}, \mathcal{M}\right\} d_{p} \mathcal{L}-\left\{\mathcal{B}_{n}, \mathcal{L}\right\} d_{p} \mathcal{M}\right) \\
& =\operatorname{Res} \mathcal{L}^{m} d_{p} \mathcal{B}_{n}
\end{aligned}
$$

where we have used the Lax equations (2.1.1), (2.3.3) and the canonical Poisson relation $\{\mathcal{L}, \mathcal{M}\}=\mathcal{L}$. The other equations of $(2.4 .7-9)$ can be proved similarly.

Now we return to the proof of Proposition 2.4.1. By the above lemma and the formula $\operatorname{Res} F d_{p} G=-\operatorname{Res} G d_{p} F$ (integration by parts), we obtain

$$
\begin{aligned}
\left.\frac{\partial S}{\partial t_{n}}\right|_{\mathcal{L}, t_{m}(m \neq n), \bar{t}, s \text { fixed }} & =\mathcal{L}^{n}-\sum_{m=1}^{\infty} \frac{1}{m} \frac{\partial v_{m}}{\partial t_{n}} \mathcal{L}^{-m} \\
& =\mathcal{L}^{n}+\sum_{m=1}^{\infty} \operatorname{Res}\left(\mathcal{B}_{n} \mathcal{L}^{m-1} d_{p} \mathcal{L}\right) \mathcal{L}^{-m}
\end{aligned}
$$

This proves the first equation of (2.4.4) due to the tautological formula

$$
A(p, s)=\sum_{n \in \mathbb{Z}} \operatorname{Res}\left(A(p, s) \mathcal{L}^{n-1} d_{p} \mathcal{L}\right) \mathcal{L}^{n} .
$$

We can prove the remaining equations of (2.4.4-6) similarly, using Lemma 2.4 .2 and Proposition 2.1.2.

We can now define a dispersionless analogue of the tau function (the free energy) of the Toda lattice hierarchy as is the case with the KP hierarchy.

Proposition 2.4.3. There exists a function $\log \tau_{\mathrm{dToda}}(t, \bar{t}, s)$ satisfying

$$
d \log \tau_{\text {dToda }}(t, \bar{t}, s)=\sum_{n=1}^{\infty} v_{n} d t_{n}-\sum_{n=1}^{\infty} \bar{v}_{n} d \bar{t}_{n}+\phi d s .
$$


Proof. We have only to check that the right hand side of (2.4.11) is a closed form, which is equivalent to the following system.

$$
\begin{gathered}
\frac{\partial v_{n}}{\partial t_{m}}=\frac{\partial v_{m}}{\partial t_{n}}, \quad \frac{\partial \bar{v}_{n}}{\partial \bar{t}_{m}}=\frac{\partial \bar{v}_{m}}{\partial \bar{t}_{n}}, \quad \frac{\partial v_{n}}{\partial \bar{t}_{m}}=\frac{\partial \bar{v}_{m}}{\partial t_{n}} \\
\frac{\partial v_{n}}{\partial s}=\frac{\partial \phi}{\partial t_{n}}, \quad \frac{\partial \bar{v}_{n}}{\partial s}=\frac{\partial \phi}{\partial \bar{t}_{n}} .
\end{gathered}
$$

This is easily proved by Lemma 2.4.2 and Proposition 2.1.2.

Like the dispersionless KP hierarchy, all ingredients $\mathcal{L}, \overline{\mathcal{L}}, \mathcal{M}, \overline{\mathcal{M}}$ etc. of the dispersionless Toda hierarchy can be thus reproduced from the single function $\log \tau_{\text {dToda }}$. We do not know yet if there exists the dispersionless analogue of the Hirota-type equations which characterize the tau function of the dispersionless Toda hierarchy.

\subsection{Riemann-Hilbert problem.}

In this section we give a construction of solutions based on the Riemann-Hilbert problem parallel to that for the dispersionless KP hierarchy in $\S 1.5$.

Proposition 2.5.1. Let two pairs of functions $(f(p, s), g(p, s)),(\bar{f}(p, s), \bar{g}(p, s))$ and two pairs of Laurent series $(\mathcal{L}, \mathcal{M}),(\overline{\mathcal{L}}, \overline{\mathcal{M}})$ be given. Suppose

(1) $(\mathcal{L}, \overline{\mathcal{L}})$ has the form (2.1.2), and $(\mathcal{M}, \overline{\mathcal{M}})$ the form (2.3.2).

(2) $(f, g)$ and $(\bar{f}, \bar{g})$ satisfy the canonical Poisson relation $\{f, g\}=f,\{\bar{f}, \bar{g}\}=\bar{f}$.

(3) $(\mathcal{L}, \mathcal{M})$ and $(\overline{\mathcal{L}}, \overline{\mathcal{M}})$ are connected by

$$
f(\mathcal{L}, \mathcal{M})=\bar{f}(\overline{\mathcal{L}}, \overline{\mathcal{M}}), \quad g(\mathcal{L}, \mathcal{M})=\bar{g}(\overline{\mathcal{L}}, \overline{\mathcal{M}})
$$

Then the quadruplet $(\mathcal{L}, \overline{\mathcal{L}}, \mathcal{M}, \overline{\mathcal{M}})$ gives a solution of the dispersionless Toda hierarchy. Namely, they satisfy the Lax equations (2.1.1), (2.3.3), and the canonical Poisson relations $\{\mathcal{L}, \mathcal{M}\}=\mathcal{L},\{\overline{\mathcal{L}}, \overline{\mathcal{M}}\}=\overline{\mathcal{L}}$. We call $(f, g, \bar{f}, \bar{g})$ the twistor data of this solution.

Proof. We follow the proof of the same construction for the dispersionless KP hierarchy 
in [1]. First we prove the canonical Poisson relations. By the chain rule we have

$$
\begin{aligned}
& \left(\begin{array}{ll}
\frac{\partial f}{\partial \mathcal{L}}(\mathcal{L}, \mathcal{M}) & \frac{\partial f}{\partial \mathcal{M}}(\mathcal{L}, \mathcal{M}) \\
\frac{\partial g}{\partial \mathcal{L}}(\mathcal{L}, \mathcal{M}) & \frac{\partial g}{\partial \mathcal{M}}(\mathcal{L}, \mathcal{M})
\end{array}\right)\left(\begin{array}{ll}
\frac{\partial \mathcal{L}}{\partial p} & \frac{\partial \mathcal{L}}{\partial s} \\
\frac{\partial \mathcal{M}}{\partial p} & \frac{\partial \mathcal{M}}{\partial s}
\end{array}\right) \\
= & \left(\begin{array}{ll}
\frac{\partial \bar{f}}{\partial \mathcal{L}}(\overline{\mathcal{L}}, \overline{\mathcal{M}}) & \frac{\partial \bar{f}}{\partial \mathcal{M}}(\overline{\mathcal{L}}, \overline{\mathcal{M}}) \\
\frac{\partial \bar{g}}{\partial \mathcal{L}}(\overline{\mathcal{L}}, \overline{\mathcal{M}}) & \frac{\partial \bar{g}}{\partial \mathcal{M}}(\overline{\mathcal{L}}, \overline{\mathcal{M}})
\end{array}\right)\left(\begin{array}{ll}
\frac{\partial \overline{\mathcal{L}}}{\partial p} & \frac{\partial \overline{\mathcal{L}}}{\partial s} \\
\frac{\partial \overline{\mathcal{M}}}{\partial p} & \frac{\partial \overline{\mathcal{M}}}{\partial s}
\end{array}\right) .
\end{aligned}
$$

Taking the determinant of the both hand sides of this equation and using the relations $\{f, g\}=f,\{\bar{f}, \bar{g}\}=\bar{f}$, we get

$$
\mathcal{L}^{-1}\{\mathcal{L}, \mathcal{M}\}=\overline{\mathcal{L}}^{-1}\{\overline{\mathcal{L}}, \overline{\mathcal{M}}\}
$$

By the assumption (1) of the proposition the right hand side of this equation is of the form $1+($ negative powers of $p)$, while the left hand side contains only the positive powers of $p$. Therefore the both hand sides of (2.5.3) are equal to 1, which proves the canonical Poisson relations.

The Lax equations with respect to $t_{n}$ are proved as follows. First, differentiating (2.5.1) by $t_{n}$ gives

$$
\left(\begin{array}{ll}
\frac{\partial f}{\partial \mathcal{L}}(\mathcal{L}, \mathcal{M}) & \frac{\partial f}{\partial \mathcal{M}}(\mathcal{L}, \mathcal{M}) \\
\frac{\partial g}{\partial \mathcal{L}}(\mathcal{L}, \mathcal{M}) & \frac{\partial g}{\partial \mathcal{M}}(\mathcal{L}, \mathcal{M})
\end{array}\right)\left(\begin{array}{c}
\frac{\partial \mathcal{L}}{\partial t_{n}} \\
\frac{\partial \mathcal{M}}{\partial t_{n}}
\end{array}\right)=\left(\begin{array}{ll}
\frac{\partial \bar{f}}{\partial \mathcal{L}}(\overline{\mathcal{L}}, \overline{\mathcal{M}}) & \frac{\partial \bar{f}}{\partial \mathcal{M}}(\overline{\mathcal{L}}, \overline{\mathcal{M}}) \\
\frac{\partial \bar{g}}{\partial \mathcal{L}}(\overline{\mathcal{L}}, \overline{\mathcal{M}}) & \frac{\partial \bar{g}}{\partial \mathcal{M}}(\overline{\mathcal{L}}, \overline{\mathcal{M}})
\end{array}\right)\left(\begin{array}{c}
\frac{\partial \overline{\mathcal{L}}}{\partial t_{n}} \\
\frac{\partial \mathcal{M}}{\partial t_{n}}
\end{array}\right)
$$

Using (2.5.2), we can rewrite (2.5.4) as

$$
\left(\begin{array}{ll}
\frac{\partial \mathcal{L}}{\partial p} & \frac{\partial \mathcal{L}}{\partial s} \\
\frac{\partial \mathcal{M}}{\partial p} & \frac{\partial \mathcal{M}}{\partial s}
\end{array}\right)^{-1}\left(\begin{array}{c}
\frac{\partial \mathcal{L}}{\partial t_{n}} \\
\frac{\partial \mathcal{M}}{\partial t_{n}}
\end{array}\right)=\left(\begin{array}{ll}
\frac{\partial \overline{\mathcal{L}}}{\partial p} & \frac{\partial \overline{\mathcal{L}}}{\partial s} \\
\frac{\partial \mathcal{M}}{\partial p} & \frac{\partial \mathcal{M}}{\partial s}
\end{array}\right)^{-1}\left(\begin{array}{c}
\frac{\partial \overline{\mathcal{L}}}{\partial t_{n}} \\
\frac{\partial \mathcal{M}}{\partial t_{n}}
\end{array}\right)
$$

The inverse matrices in this formula are easily calculated because of the canonical Poisson relations. Thus we get

$$
\mathcal{L}^{-1}\left(\begin{array}{cc}
\frac{\partial \mathcal{M}}{\partial s} & -\frac{\partial \mathcal{L}}{\partial s} \\
-\frac{\partial \mathcal{M}}{\partial p} & \frac{\partial \mathcal{L}}{\partial p}
\end{array}\right)\left(\begin{array}{c}
\frac{\partial \mathcal{L}}{\partial t_{n}} \\
\frac{\partial \mathcal{M}}{\partial t_{n}}
\end{array}\right)=\overline{\mathcal{L}}^{-1}\left(\begin{array}{cc}
\frac{\partial \overline{\mathcal{M}}}{\partial s} & -\frac{\partial \overline{\mathcal{L}}}{\partial s} \\
-\frac{\partial \mathcal{M}}{\partial p} & \frac{\partial \mathcal{L}}{\partial p}
\end{array}\right)\left(\begin{array}{c}
\frac{\partial \overline{\mathcal{L}}}{\partial t_{n}} \\
\frac{\partial \mathcal{M}}{\partial t_{n}}
\end{array}\right)
$$


The first component of the right hand side is given by

$$
\begin{aligned}
& \overline{\mathcal{L}}^{-1}\left(\frac{\partial \overline{\mathcal{M}}}{\partial s} \frac{\partial \overline{\mathcal{L}}}{\partial t_{n}}-\frac{\partial \overline{\mathcal{M}}}{\partial t_{n}} \frac{\partial \overline{\mathcal{L}}}{\partial s}\right) \\
= & \overline{\mathcal{L}}^{-1}\left(\left.\frac{\partial \overline{\mathcal{M}}}{\partial \overline{\mathcal{L}}}\right|_{t, v \text { fixed }} \frac{\partial \overline{\mathcal{L}}}{\partial s}+1-\sum_{n=1}^{\infty} \frac{\partial \bar{v}_{n}}{\partial s} \overline{\mathcal{L}}^{n}\right) \frac{\partial \overline{\mathcal{L}}}{\partial t_{n}} \\
- & \overline{\mathcal{L}}^{-1}\left(\left.\frac{\partial \overline{\mathcal{M}}}{\partial \overline{\mathcal{L}}}\right|_{t, v \text { fixed }} \frac{\partial \overline{\mathcal{L}}}{\partial t_{n}}+\sum_{n=1}^{\infty} \frac{\partial \bar{v}_{n}}{\partial t_{n}} \overline{\mathcal{L}}^{n}\right) \frac{\partial \overline{\mathcal{L}}}{\partial s} \\
= & \overline{\mathcal{L}}^{-1}\left(1-\sum_{n=1}^{\infty} \frac{\partial \bar{v}_{n}}{\partial s} \overline{\mathcal{L}}^{n}\right) \frac{\partial \overline{\mathcal{L}}}{\partial t_{n}}-\overline{\mathcal{L}}^{-1}\left(\sum_{n=1}^{\infty} \frac{\partial \bar{v}_{n}}{\partial t_{n}} \overline{\mathcal{L}}^{n}\right) \frac{\partial \overline{\mathcal{L}}}{\partial s}
\end{aligned}
$$

which does not contain negative powers of $p$. Similarly the first component of the left hand side of (2.5.6) is given by

$$
\mathcal{L}^{-1}\left(\frac{\partial \mathcal{M}}{\partial s} \frac{\partial \mathcal{L}}{\partial t_{n}}-\frac{\partial \mathcal{M}}{\partial t_{n}} \frac{\partial \mathcal{L}}{\partial s}\right)=-\frac{\partial\left(\mathcal{L}^{n}\right)}{\partial s}+\text { negative powers of } p
$$

Comparing (2.5.7) and (2.5.8), we obtain

$$
\mathcal{L}^{-1}\left(\frac{\partial \mathcal{M}}{\partial s} \frac{\partial \mathcal{L}}{\partial t_{n}}-\frac{\partial \mathcal{M}}{\partial t_{n}} \frac{\partial \mathcal{L}}{\partial s}\right)=\overline{\mathcal{L}}^{-1}\left(\frac{\partial \overline{\mathcal{M}}}{\partial s} \frac{\partial \overline{\mathcal{L}}}{\partial t_{n}}-\frac{\partial \overline{\mathcal{M}}}{\partial t_{n}} \frac{\partial \overline{\mathcal{L}}}{\partial s}\right)=-\frac{\partial \mathcal{B}_{n}}{\partial s}
$$

By the same method, we obtain from the second component of (2.5.6)

$$
\mathcal{L}^{-1}\left(\frac{\partial \mathcal{M}}{\partial s} \frac{\partial \mathcal{L}}{\partial t_{n}}-\frac{\partial \mathcal{M}}{\partial t_{n}} \frac{\partial \mathcal{L}}{\partial s}\right)=\overline{\mathcal{L}}^{-1}\left(\frac{\partial \overline{\mathcal{M}}}{\partial s} \frac{\partial \overline{\mathcal{L}}}{\partial t_{n}}-\frac{\partial \overline{\mathcal{M}}}{\partial t_{n}} \frac{\partial \overline{\mathcal{L}}}{\partial s}\right)=\frac{\partial \mathcal{B}_{n}}{\partial p}
$$

Equations (2.5.9) and (2.5.10) reduce to a linear equation

$$
\mathcal{L}^{-1}\left(\begin{array}{cc}
\frac{\partial \mathcal{M}}{\partial s} & -\frac{\partial \mathcal{L}}{\partial s} \\
-\frac{\partial \mathcal{M}}{\partial p} & \frac{\partial \mathcal{L}}{\partial p}
\end{array}\right)\left(\begin{array}{l}
\frac{\partial \mathcal{L}}{\partial t_{n}} \\
\frac{\partial \mathcal{M}}{\partial t_{n}}
\end{array}\right)=\overline{\mathcal{L}}^{-1}\left(\begin{array}{cc}
\frac{\partial \overline{\mathcal{M}}}{\partial s} & -\frac{\partial \overline{\mathcal{L}}}{\partial s} \\
-\frac{\partial \overline{\mathcal{M}}}{\partial p} & \frac{\partial \overline{\mathcal{L}}}{\partial p}
\end{array}\right)\left(\begin{array}{c}
\frac{\partial \overline{\mathcal{L}}}{\partial t_{n}} \\
\frac{\partial \mathcal{M}}{\partial t_{n}}
\end{array}\right)=\left(\begin{array}{c}
-\frac{\partial \mathcal{B}_{n}}{\partial s} \\
\frac{\partial \mathcal{B}_{n}}{\partial p}
\end{array}\right)
$$

Again due to the canonical Poisson relations, the inverse of the matrices in (2.5.11) can be explicitly calculated, and we obtain

$$
\left(\begin{array}{c}
\frac{\partial \mathcal{L}}{\partial t_{n}} \\
\frac{\partial \mathcal{M}}{\partial t_{n}}
\end{array}\right)=\left(\begin{array}{c}
\left\{\mathcal{B}_{n}, \mathcal{L}\right\} \\
\left\{\mathcal{B}_{n}, \mathcal{M}\right\}
\end{array}\right), \quad\left(\begin{array}{c}
\frac{\partial \overline{\mathcal{L}}}{\partial t_{n}} \\
\frac{\partial \mathcal{\mathcal { M }}}{\partial t_{n}}
\end{array}\right)=\left(\begin{array}{c}
\left\{\mathcal{B}_{n}, \overline{\mathcal{L}}\right\} \\
\left\{\mathcal{B}_{n}, \overline{\mathcal{M}}\right\}
\end{array}\right)
$$

which is nothing but $t$-flow part of the Lax equations (2.1.1) and (2.3.3). The $\bar{t}$-flow part of the Lax equations can be proved in the same way. 
This construction is a kind of Riemann-Hilbert decomposition:

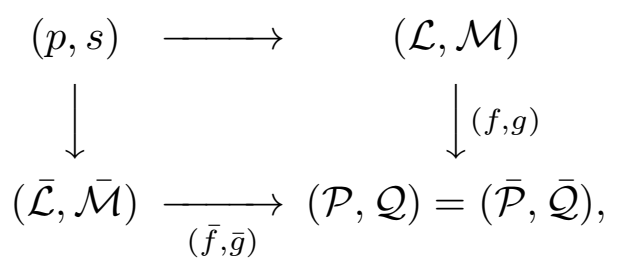

where $(\mathcal{P}, \mathcal{Q})=(f(\mathcal{L}, \mathcal{M}), g(\mathcal{L}, \mathcal{M})), \quad(\overline{\mathcal{P}}, \overline{\mathcal{Q}})=(\bar{f}(\overline{\mathcal{L}}, \overline{\mathcal{M}}), \bar{g}(\overline{\mathcal{L}}, \overline{\mathcal{M}}))$. The areapreserving map from the right upper corner to the left lower corner of this diagram (the composition of $(f, g)$ and the inverse map of $(\bar{f}, \bar{g}))$ is decomposed into two maps, $(\mathcal{L}, \mathcal{M}) \mapsto(p, s)$ and $(p, s) \mapsto(\overline{\mathcal{L}}, \overline{\mathcal{M}})$. The former of these two maps is rationally extended to $p=\infty$, while the latter is extended to $p=0$.

The existence of the twistor data for solutions is ensured as follows.

Proposition 2.5.2. Let $(\mathcal{L}, \overline{\mathcal{L}}, \mathcal{M}, \overline{\mathcal{M}})$ be a solution of the dispersionless Toda hierarchy. Then there exist twistor data $(f, g, \bar{f}, \bar{g})$ which satisfy (2) and (3) of Proposition 2.5.1.

Proof. Let $\varphi, \bar{\varphi}$ be dressing operators in the sense of $\S 2.2$. Then

$$
\begin{array}{ll}
f(p, s)=e^{-\operatorname{ad} \varphi(t=\bar{t}=0, p, s)} p, & g(p, s)=e^{-\operatorname{ad} \varphi(t=\bar{t}=0, p, s)} s, \\
\bar{f}(p, s)=e^{-\operatorname{ad} \bar{\varphi}(t=\bar{t}=0, p, s)} e^{-\operatorname{ad} \phi(t=\bar{t}=0, s)} p, & \bar{g}(p, s)=e^{-\operatorname{ad} \bar{\varphi}(t=\bar{t}=0, p, s)} e^{-\operatorname{ad} \phi(t=\bar{t}=0, s)} s,
\end{array}
$$

are the corresponding twistor data. We omit the proof, which is based on the unique solvability of the initial value problem satisfied by the both hand sides of (2.5.1) (cf. the proof of Proposition 1.5.2).

\section{6. $w_{1+\infty}$ symmetry.}

Here we describe $w_{1+\infty}$ symmetries of the dispersionless Toda hierarchy induced by deformation of the twistor data. As in $§ 1.6$ we consider infinitesimal deformation of $(f, g)$ and $(\bar{f}, \bar{g})(f \neq 0, \bar{f} \neq 0)$ by Hamiltonian vector fields:

$$
\begin{aligned}
(f, g) & \mapsto(f, g) \circ \exp (-\varepsilon \operatorname{ad} F), \\
(\bar{f}, \bar{g}) & \mapsto(\bar{f}, \bar{g}) \circ \exp (-\varepsilon \operatorname{ad} \bar{F}),
\end{aligned}
$$


and the associated deformation

$$
\begin{aligned}
(\mathcal{L}, \mathcal{M}) & \mapsto(\mathcal{L}(\varepsilon), \mathcal{M}(\varepsilon)), \\
(\overline{\mathcal{L}}, \overline{\mathcal{M}}) & \mapsto(\overline{\mathcal{L}}(\varepsilon), \overline{\mathcal{M}}(\varepsilon))
\end{aligned}
$$

of the $(\mathcal{L}, \mathcal{M})$-pair and the $(\overline{\mathcal{L}}, \overline{\mathcal{M}})$-pair. Here ad $F$ and ad $\bar{F}$ are regarded as Hamiltonian vector fields:

$$
\text { ad } F=p \frac{\partial F}{\partial p} \frac{\partial}{\partial s}-p \frac{\partial F}{\partial s} \frac{\partial}{\partial p}, \quad \text { ad } \bar{F}=p \frac{\partial \bar{F}}{\partial p} \frac{\partial}{\partial s}-p \frac{\partial \bar{F}}{\partial s} \frac{\partial}{\partial p},
$$

and $\varepsilon$ an infinitesimal parameter. The rule of the game is the same as in $§ 1.6$. Infinitesimal symmetry is defined as the first order coefficient $\delta_{F, \bar{F}}$ in $\varepsilon$-expansion:

$$
\begin{aligned}
\mathcal{L}(\varepsilon) & =\mathcal{L}+\varepsilon \delta_{F, \bar{F}} \mathcal{L}+O\left(\varepsilon^{2}\right), & \overline{\mathcal{L}}(\varepsilon) & =\overline{\mathcal{L}}+\varepsilon \delta_{F, \bar{F}} \overline{\mathcal{L}}+O\left(\varepsilon^{2}\right), \\
\mathcal{M}(\varepsilon) & =\mathcal{M}+\varepsilon \delta_{F, \bar{F}} \mathcal{M}+O\left(\varepsilon^{2}\right), & \overline{\mathcal{M}}(\varepsilon) & =\overline{\mathcal{M}}+\varepsilon \delta_{F, \bar{F}} \overline{\mathcal{M}}+O\left(\varepsilon^{2}\right)
\end{aligned}
$$

By definition, $\delta_{F, \bar{F}}$ acts on any functions of $\mathcal{L}, \mathcal{M}, \overline{\mathcal{L}}, \overline{\mathcal{M}}$ as derivation (cf. (1.6.2)), and leave the independent variables invariant:

$$
\delta_{F, \bar{F}} t_{n}=\delta_{F, \bar{F}} \bar{t}_{n}=\delta_{F, \bar{F}} p=\delta_{F, \bar{F}} s=0 .
$$

Proposition 2.6.1. i) The infinitesimal symmetries of $\mathcal{L}, \mathcal{M}, \overline{\mathcal{L}}, \overline{\mathcal{M}}$ are given by

$$
\begin{aligned}
\delta_{F, \bar{F}} \mathcal{L} & =\left\{F(\mathcal{L}, \mathcal{M})_{\leq-1}-\bar{F}(\overline{\mathcal{L}}, \overline{\mathcal{M}})_{\leq-1}, \mathcal{L}\right\}, \\
\delta_{F, \bar{F}} \mathcal{M} & =\left\{F(\mathcal{L}, \mathcal{M})_{\leq-1}-\bar{F}(\overline{\mathcal{L}}, \overline{\mathcal{M}})_{\leq-1}, \mathcal{M}\right\}, \\
\delta_{F, \bar{F}} \overline{\mathcal{L}} & =\left\{\bar{F}(\overline{\mathcal{L}}, \overline{\mathcal{M}})_{\geq 0}-F(\mathcal{L}, \mathcal{M})_{\geq 0}, \overline{\mathcal{L}}\right\}, \\
\delta_{F, \bar{F}} \overline{\mathcal{M}} & =\left\{\bar{F}(\overline{\mathcal{L}}, \overline{\mathcal{M}})_{\geq 0}-F(\mathcal{L}, \mathcal{M})_{\geq 0}, \overline{\mathcal{M}}\right\} .
\end{aligned}
$$

ii) The induced symmetries of $v_{i}, i=1,2, \ldots$ are given by

$$
\begin{aligned}
\delta_{F, \bar{F}} v_{i} & =-\operatorname{Res}(F(\mathcal{L}, \mathcal{M})-\bar{F}(\overline{\mathcal{L}}, \overline{\mathcal{M}})) d_{p} \mathcal{B}_{i}, \\
\delta_{F, \bar{F}} \bar{v}_{i} & =-\operatorname{Res}(F(\mathcal{L}, \mathcal{M})-\bar{F}(\overline{\mathcal{L}}, \overline{\mathcal{M}})) d_{p} \overline{\mathcal{B}}_{i} .
\end{aligned}
$$

iii) The induced symmetries of the dressing functions are given by

$$
\begin{aligned}
\nabla_{\delta_{F, \bar{F}}, \varphi} \varphi & =F(\mathcal{L}, \mathcal{M})_{\leq-1}-\bar{F}(\overline{\mathcal{L}}, \overline{\mathcal{M}})_{\leq-1}, \\
e^{\operatorname{ad} \phi} \nabla_{\delta_{F, \bar{F}}, \bar{\varphi}} \bar{\varphi} & =\bar{F}(\overline{\mathcal{L}}, \overline{\mathcal{M}})_{\geq 0}-F(\mathcal{L}, \mathcal{M})_{\geq 0} .
\end{aligned}
$$


iv) An infinitesimal symmetry of $\phi$ consistent with i) - iii) is given by

$$
\delta_{F, \bar{F}} \phi=-\operatorname{Res} F(\mathcal{L}, \mathcal{M}) d \log p+\operatorname{Res} \bar{F}(\overline{\mathcal{L}}, \overline{\mathcal{M}}) d \log p .
$$

Proof. In this proof, we abbriviate $\delta_{F, \bar{F}}$ to $\delta$. Part i) is a corollary of part iii) and part iv) due to Lemma A.1. Part ii) is proved as follows. From the expansion (2.3.2) the deformation of $\mathcal{M}$ has a form

$$
\delta \mathcal{M}=\left.\frac{\partial \mathcal{M}}{\partial \mathcal{L}}\right|_{t, \bar{t}, v \text { fixed }} \delta \mathcal{L}+\sum_{i=1}^{\infty} \delta v_{i} \mathcal{L}^{-i} .
$$

The coefficient of $\mathcal{L}^{-i}$ can be thus written

$$
\begin{aligned}
\delta v_{i}= & \operatorname{Res}\left(\delta \mathcal{M}-\left.\frac{\partial \mathcal{M}}{\partial \mathcal{L}}\right|_{t, \bar{t}, v \text { fixed }}\right) \mathcal{L}^{i-1} d_{p} \mathcal{L} \\
= & \operatorname{Res} \mathcal{L}^{i-1}\left(\delta \mathcal{M} d_{p} \mathcal{L}-\delta \mathcal{L} d_{p} \mathcal{M}\right) \\
= & \operatorname{Res} \mathcal{L}^{i-1}\left(\left\{F(\mathcal{L}, \mathcal{M})_{\leq-1}-\bar{F}(\overline{\mathcal{L}}, \overline{\mathcal{M}})_{\leq-1}, \mathcal{M}\right\} d_{p} \mathcal{L}\right. \\
& \left.\quad-\left\{F(\mathcal{L}, \mathcal{M})_{\leq-1}-\bar{F}(\overline{\mathcal{L}}, \overline{\mathcal{M}})_{\leq-1}, \mathcal{L}\right\} d_{p} \mathcal{M}\right)
\end{aligned}
$$

where we have used i). Expanding the Poisson brackets on the right hand side of (2.6.10), and using the relation $\{\mathcal{L}, \mathcal{M}\}=\mathcal{L}$, we can rewrite the last expression as

$$
\begin{aligned}
& \operatorname{Res} \mathcal{L}^{i-1}\left(\frac{\partial}{\partial p}\left(F(\mathcal{L}, \mathcal{M})_{\leq-1}-\bar{F}(\overline{\mathcal{L}}, \overline{\mathcal{M}})_{\leq-1}\right) \mathcal{L} d p\right) \\
= & \operatorname{Res} \mathcal{L}^{i} d_{p}\left(F(\mathcal{L}, \mathcal{M})_{\leq-1}-\bar{F}(\overline{\mathcal{L}}, \overline{\mathcal{M}})_{\leq-1}\right) \\
= & -\operatorname{Res}(F(\mathcal{L}, \mathcal{M})-\bar{F}(\overline{\mathcal{L}}, \overline{\mathcal{M}})) d_{p} \mathcal{B}_{i},
\end{aligned}
$$

which proves the first equation of (2.6.6). The second equation of (2.6.6) can be proved in the same way.

The last two statements iii) and iv) are proved as follows. Let us denote the deformed twistor data (2.6.1) by $\left(f_{\varepsilon}, g_{\varepsilon}\right),\left(\bar{f}_{\varepsilon}, \bar{g}_{\varepsilon}\right)$ and the deformation of the dressing functions $\varphi$, $\bar{\varphi}$ and the potential $\phi$ by $\varphi_{\varepsilon}=\varphi+\varepsilon \delta \varphi+O\left(\varepsilon^{2}\right), \bar{\varphi}_{\varepsilon}=\bar{\varphi}+\varepsilon \delta \bar{\varphi}+O\left(\varepsilon^{2}\right), \phi_{\varepsilon}=\phi+\varepsilon \delta \phi+O\left(\varepsilon^{2}\right)$. The Riemann-Hilbert problem (2.5.1) for the deformed twistor data becomes

$$
\begin{aligned}
& e^{\operatorname{ad} \varphi_{\varepsilon}} e^{\operatorname{ad} t(p)} f_{\varepsilon}(p, s)=e^{\operatorname{ad} \phi_{\varepsilon}} e^{\operatorname{ad} \bar{\varphi}_{\varepsilon}} e^{\operatorname{ad} \bar{t}\left(p^{-1}\right)} \bar{f}_{\varepsilon}(p, s), \\
& e^{\operatorname{ad} \varphi_{\varepsilon}} e^{\operatorname{ad} t(p)} g_{\varepsilon}(p, s)=e^{\operatorname{ad} \phi_{\varepsilon}} e^{\operatorname{ad} \bar{\varphi}_{\varepsilon}} e^{\operatorname{ad} \bar{t}\left(p^{-1}\right)} \bar{g}_{\varepsilon}(p, s) .
\end{aligned}
$$


Calculating exactly in the same manner as in the proof of Proposition 1.6.3, we obtain from the coefficient of $\varepsilon$ in $(2.6 .12)$

$$
\begin{aligned}
& \left\{\nabla_{\delta, \varphi} \varphi-F(\mathcal{L}, \mathcal{M}), \mathcal{P}\right\}=\left\{\nabla_{\delta, \phi} \phi+e^{\operatorname{ad} \phi}\left(\delta_{\delta, \bar{\varphi}} \bar{\varphi}\right)-\bar{F}(\overline{\mathcal{L}}, \overline{\mathcal{M}}), \overline{\mathcal{P}}\right\}, \\
& \left\{\nabla_{\delta, \varphi} \varphi-F(\mathcal{L}, \mathcal{M}), \mathcal{Q}\right\}=\left\{\nabla_{\delta, \phi} \phi+e^{\operatorname{ad} \phi}\left(\delta_{\delta, \bar{\varphi}} \bar{\varphi}\right)-\bar{F}(\overline{\mathcal{L}}, \overline{\mathcal{M}}), \overline{\mathcal{Q}}\right\}
\end{aligned}
$$

where $\mathcal{P}=f(\mathcal{L}, \mathcal{M}), \mathcal{Q}=g(\mathcal{L}, \mathcal{M}), \overline{\mathcal{P}}=\bar{f}(\overline{\mathcal{L}}, \overline{\mathcal{M}}), \overline{\mathcal{Q}}=\bar{g}(\overline{\mathcal{L}}, \overline{\mathcal{M}})$. Since $(\mathcal{L}, \mathcal{M}, \overline{\mathcal{L}}, \overline{\mathcal{M}})$ is a solution of $(2.5 .1)$, we have $\mathcal{P}=\overline{\mathcal{P}}, \mathcal{Q}=\overline{\mathcal{Q}}$, and $\{\mathcal{P}, \mathcal{Q}\}=\mathcal{P},\{\overline{\mathcal{P}}, \overline{\mathcal{Q}}\}=\overline{\mathcal{P}}$. Hence the following Lemma 2.6.2 proves iii) (2.6.7). To prove iv) note that $\nabla_{\delta, \phi} \phi=\delta \phi$. This follows from the fact that $\phi$ does not contain $p$. Hence (2.6.13) and Lemma 2.6.2 below imply

$$
\delta \phi=-\operatorname{Res} F(\mathcal{L}, \mathcal{M}) d \log p+\operatorname{Res} \bar{F}(\overline{\mathcal{L}}, \overline{\mathcal{M}}) d \log p+\text { const. }
$$

where const. denotes a term which does not depend on $(p, s)$. Thus this ambiguity belongs to the center of the Lie algebra of Poisson brackets, from which iv) follows.

Lemma 2.6.2. Suppose that $\mathcal{P} \neq 0$ and $\mathcal{Q}$ satisfy the canonical Poisson relation $\{\mathcal{P}, \mathcal{Q}\}=\mathcal{P}$, and that a series $\mathcal{A}$ satisfy

$$
\{\mathcal{A}, \mathcal{P}\}=\{\mathcal{A}, \mathcal{Q}\}=0
$$

Then $\partial \mathcal{A} / \partial p=\partial \mathcal{A} / \partial s=0$.

Proof. The equation (2.6.15) is equivalent to

$$
\left(\begin{array}{ll}
\frac{\partial \mathcal{P}}{\partial s} & -\frac{\partial \mathcal{P}}{\partial p} \\
\frac{\partial \mathcal{Q}}{\partial s} & -\frac{\partial \mathcal{Q}}{\partial p}
\end{array}\right)\left(\begin{array}{l}
\frac{\partial \mathcal{A}}{\partial p} \\
\frac{\partial \mathcal{A}}{\partial s}
\end{array}\right)=0
$$

Because of the canonical Poisson relation, the determinant of the matrix on the left hand side of (2.6.16) is $p^{-1} \mathcal{P}$ which does not vanish by the assumption. This proves the lemma.

This symmetry can be lifted up to the tau function as follows. 
Proposition 2.6.3. An infinitesimal symmetry of $\tau=\tau_{\mathrm{dToda}}$ consistent with $i$ ) - iii) is given by

$$
\delta_{F, \bar{F}} \log \tau=-\operatorname{Res} F^{s}(\mathcal{L}, \mathcal{M}) d_{p} \log \mathcal{L}+\operatorname{Res} \bar{F}^{s}(\overline{\mathcal{L}}, \overline{\mathcal{M}}) d_{p} \log \overline{\mathcal{L}}
$$

where $F^{s}$ and $\bar{F}^{s}$ are defined by

$$
F^{s}(p, s) \underset{\operatorname{def}}{=} \int_{0}^{s} F\left(p, s^{\prime}\right) d s^{\prime}, \quad \bar{F}^{s}(p, s) \underset{\operatorname{def}}{=} \int_{0}^{s} \bar{F}\left(p, s^{\prime}\right) d s^{\prime} .
$$

Proof. The consistency means

$$
\begin{gathered}
\frac{\partial}{\partial t_{n}} \delta \log \tau=\delta \frac{\partial \log \tau}{\partial t_{n}}, \quad \frac{\partial}{\partial \bar{t}_{n}} \delta \log \tau=\delta \frac{\partial \log \tau}{\partial \bar{t}_{n}} \\
\frac{\partial}{\partial s} \delta \log \tau=\delta \frac{\partial \log \tau}{\partial s}
\end{gathered}
$$

where $\delta=\delta_{F, \bar{F}}$ as before.

First let us consider (2.6.19). We check here only the first equation of (2.6.19). The second equation of (2.6.19) can be checked similarly. The right hand side of this equation has been already calculated in Proposition 2.6.1 ii):

$$
\delta \frac{\partial \log \tau}{\partial t_{n}}=\delta v_{n}=-\operatorname{Res}(F(\mathcal{L}, \mathcal{M})-\bar{F}(\overline{\mathcal{L}}, \overline{\mathcal{M}})) d_{p} \mathcal{B}_{n}
$$

The left hand side of (2.6.19) is written as

$$
\frac{\partial}{\partial t_{n}} \delta \log \tau=-\frac{\partial}{\partial t_{n}} \operatorname{Res} F^{s}(\mathcal{L}, \mathcal{M}) d_{p} \log \mathcal{L}+\frac{\partial}{\partial t_{n}} \operatorname{Res} \bar{F}^{s}(\overline{\mathcal{L}}, \overline{\mathcal{M}}) d_{p} \log \overline{\mathcal{L}}
$$

The terms of the right hand side of (2.6.22) can be calculated exactly in the same way as the corresponding expression of the dispersionless KP hierarchy ([1] Proposition 15). Therefore we omit the details of calculation and give only the result:

$$
\begin{aligned}
& \frac{\partial}{\partial t_{n}} \operatorname{Res} F^{s}(\mathcal{L}, \mathcal{M}) d_{p} \log \mathcal{L}=\operatorname{Res} F(\mathcal{L}, \mathcal{M}) d_{p} \mathcal{B}_{n} \\
& \frac{\partial}{\partial t_{n}} \operatorname{Res} \bar{F}^{s}(\overline{\mathcal{L}}, \overline{\mathcal{M}}) d_{p} \log \mathcal{L}=\operatorname{Res} \bar{F}(\overline{\mathcal{L}}, \overline{\mathcal{M}}) d_{p} \mathcal{B}_{n}
\end{aligned}
$$


Thus (2.6.21) and (2.6.22) give the identical results, which proves (2.6.19).

We turn to the proof of equation (2.6.20). The right hand side of this equation is

$$
\delta \frac{\partial \log \tau}{\partial s}=\delta \phi=-\operatorname{Res} F(\mathcal{L}, \mathcal{M}) d \log p+\operatorname{Res} \bar{F}(\overline{\mathcal{L}}, \overline{\mathcal{M}}) d \log p
$$

because of (2.6.8). The left hand side of (2.6.20) is calculated by the same method as $(2.6 .23)$ :

$$
\begin{aligned}
\frac{\partial}{\partial s} \delta \log \tau= & \frac{\partial}{\partial s}\left(-\operatorname{Res} F^{s}(\mathcal{L}, \mathcal{M}) d_{p} \log \mathcal{L}+\operatorname{Res} \bar{F}^{s}(\overline{\mathcal{L}}, \overline{\mathcal{M}}) d_{p} \log \overline{\mathcal{L}}\right) \\
= & -\operatorname{Res} F(\mathcal{L}, \mathcal{M})\left(\frac{\partial \mathcal{M}}{\partial s}-\left.\frac{\partial \mathcal{M}}{\partial \mathcal{L}}\right|_{t, \bar{t}, v \text { fixed }} \frac{\partial \mathcal{L}}{\partial s}\right) d_{p} \log \mathcal{L} \\
& \left.+\operatorname{Res} \bar{F}(\overline{\mathcal{L}}, \overline{\mathcal{M}})\left(\frac{\partial \overline{\mathcal{M}}}{\partial s}-\left.\frac{\partial \overline{\mathcal{M}}}{\partial \overline{\mathcal{L}}}\right|_{t, \bar{t}, v \text { fixed }} \frac{\partial \overline{\mathcal{L}}}{\partial s}\right) d_{p} \log \overline{\mathcal{L}}\right) \\
= & -\operatorname{Res} F(\mathcal{L}, \mathcal{M}) \mathcal{L}^{-1}\left(\frac{\partial \mathcal{M}}{\partial s} \frac{\partial \mathcal{L}}{\partial p}-\frac{\partial \mathcal{M}}{\partial p} \frac{\partial \mathcal{L}}{\partial s}\right) d p \\
& +\operatorname{Res} \bar{F}(\overline{\mathcal{L}}, \overline{\mathcal{M}}) \overline{\mathcal{L}}^{-1}\left(\frac{\partial \overline{\mathcal{M}}}{\partial s} \frac{\partial \overline{\mathcal{L}}}{\partial p}-\frac{\partial \mathcal{\mathcal { M }}}{\partial p} \frac{\partial \overline{\mathcal{L}}}{\partial s}\right) d p \\
= & -\operatorname{Res} F(\mathcal{L}, \mathcal{M}) p^{-1} d p+\operatorname{Res} \bar{F}(\overline{\mathcal{L}}, \overline{\mathcal{M}}) p^{-1} d p
\end{aligned}
$$

by the canonical Poisson relations. Equations (2.6.24), (2.6.25) prove the equation $(2.6 .20)$.

These infinitesimal symmetries almost respect the $w_{1+\infty}$ algebra structure as follows.

Proposition 2.6.4. i) Commutation relations for $\delta_{F, \bar{F}}$ on $\mathcal{L}, \mathcal{M}, \overline{\mathcal{L}}, \overline{\mathcal{M}}, \varphi, \bar{\varphi}$ and $\phi$ have the form;

$$
\left[\delta_{F_{1}, \bar{F}_{1}}, \delta_{F_{2}, \bar{F}_{2}}\right] K=\delta_{\left\{F_{1}, F_{2}\right\},\left\{\bar{F}_{1}, \bar{F}_{2}\right\}} K
$$

where $K$ is one of $\mathcal{L}, \mathcal{M}, \overline{\mathcal{L}}, \overline{\mathcal{M}}, \varphi, \bar{\varphi}$ and $\phi$.

ii) For the tau function,

$$
\left[\delta_{F_{1}, \bar{F}_{1}}, \delta_{F_{2}, \bar{F}_{2}}\right] \log \tau=\delta_{\left\{F_{1}, F_{2}\right\},\left\{\bar{F}_{1}, \bar{F}_{2}\right\}} \log \tau+c\left(F_{1}, F_{2}\right)+\bar{c}\left(\bar{F}_{1}, \bar{F}_{2}\right),
$$

where $c$ and $\bar{c}$ are cocycles of the $w_{1+\infty}$-algebra given by

$$
\begin{aligned}
& c\left(F_{1}, F_{2}\right) \underset{\text { def }}{=}-\operatorname{Res} F_{2}(p, 0) d F_{1}(p, 0), \\
& \bar{c}\left(\bar{F}_{1}, \bar{F}_{2}\right) \underset{\text { def }}{=} \operatorname{Res} \bar{F}_{2}(p, 0) d \bar{F}_{1}(p, 0) .
\end{aligned}
$$


Proof. The commutation relation for $\mathcal{L}, \mathcal{M}, \overline{\mathcal{L}}, \overline{\mathcal{M}}, \varphi, \bar{\varphi}$ and $\phi$ is derived from that for the tau function due to consistency in Proposition 2.6.3 and (2.4.11), (2.3.4).

It is sufficient to prove the commutation relation for the tau function (2.6.28) only for the case that $F_{i}(p, s), \bar{F}_{i}(p, s)(i=1,2)$ are monomials of $s: F_{i}(p, s)=f_{i}(p) s^{j_{i}}$,

$\bar{F}_{i}(p, s)=\bar{f}_{i}(p) s^{k_{i}}$. We omit the proof of this fact, since it is essentially the same calculation as that of the corresponding statement for the dispersionless KP hierarchy ([1], Proposition 16).

\subsection{Quasi-classical limit of Toda lattice hierarchy.}

In this section we consider the "dispersionful" counterpart of the contents of the preceding sections. Following [4], we introduce a "Planck constant" $\hbar$ into the ordinary Toda lattice hierarchy [21] and consider the limit $\hbar \rightarrow 0$. This small parameter $\hbar$ plays the role of lattice spacing in the Toda lattice. Leading terms in this limit recover the dispersionless Toda hierarchy. As in $§ 1.7$, the subsections $2.7 .1-2.7 .6$ correspond to $\S \S 2.1-2.6$.

2.7.1 Lax formalism. To interpret the dispersionless Toda hierarchy as a quasiclassical limit, we reformulate the Toda lattice hierarchy in the language of difference operators of an continuous variable $s$ with spacing unit $\hbar$ : $\exp (m \hbar \partial / \partial s) f(s)=f(s+m \hbar)$. The Lax representation of this reformulated Toda lattice hierarchy is given by

$$
\begin{aligned}
& \hbar \frac{\partial L}{\partial t_{n}}=\left[B_{n}, L\right], \quad \hbar \frac{\partial L}{\partial \bar{t}_{n}}=\left[\bar{B}_{n}, L\right], \\
& \hbar \frac{\partial \bar{L}}{\partial t_{n}}=\left[B_{n}, \bar{L}\right], \quad \hbar \frac{\partial \bar{L}}{\partial \bar{t}_{n}}=\left[\bar{B}_{n}, \bar{L}\right], \quad n=1,2, \ldots,
\end{aligned}
$$

where the Lax operators $L$ and $\bar{L}$ are difference operators of the form

$$
\begin{aligned}
L & =e^{\hbar \partial / \partial s}+\sum_{n=0}^{\infty} u_{n+1}(\hbar, t, \bar{t}, s) e^{-n \hbar \partial / \partial s} \\
\bar{L}^{-1} & =\bar{u}_{0}(\hbar, t, \bar{t}, s) e^{-\hbar \partial / \partial s}+\sum_{n=0}^{\infty} \bar{u}_{n+1}(\hbar, t, \bar{t}, s) e^{n \hbar \partial / \partial s}
\end{aligned}
$$

and $B_{n}$ and $\bar{B}_{n}$ are given by

$$
B_{n}=\left(L^{n}\right)_{\geq 0}, \quad \bar{B}_{n}=\left(\bar{L}^{-n}\right)_{\leq-1} .
$$


Also here, we define $\bar{u}_{n+1}$ to be the coefficients of $\bar{L}^{-1}$ rather than $\bar{L}$ itself (cf. (2.1.2)). Here ()$_{\geq 0}$ and ()$_{\leq-1}$ denote the projection onto a linear combination of $e^{n \hbar \partial / \partial s}$ with $n \geqq 0$ and $\leqq-1$, respectively. The coefficients $u_{n}, \bar{u}_{n}$ of $L, \bar{L}$ are assumed to be regular with respect to $\hbar: u_{n}(\hbar, t, \bar{t}, s)=u_{n}^{0}(t, \bar{t}, s)+O(\hbar), \bar{u}_{n}(\hbar, t, \bar{t}, s)=\bar{u}_{n}^{0}(t, \bar{t}, s)+O(\hbar)$.

As is well known, the Lax representation is equivalent to the zero-curvature representation

$$
\begin{aligned}
& \hbar \partial_{t_{n}} B_{m}-\hbar \partial_{t_{m}} B_{n}+\left[B_{m}, B_{n}\right]=0, \\
& \hbar \partial_{\bar{t}_{n}} \bar{B}_{m}-\hbar \partial_{\bar{t}_{m}} \bar{B}_{n}+\left[\bar{B}_{m}, \bar{B}_{n}\right]=0, \\
& \hbar \partial_{\bar{t}_{n}} B_{m}-\hbar \partial_{t_{m}} \bar{B}_{n}+\left[B_{m}, \bar{B}_{n}\right]=0,
\end{aligned}
$$

The "order" and the "principal symbol" are defined for the difference operators as follows.

Definition 2.7.1.

$$
\operatorname{ord}^{\hbar}\left(\sum a_{n, m}(t, \bar{t}, s) \hbar^{n} e^{m \hbar \partial / \partial s}\right) \underset{\text { def }}{=} \max \left\{n \mid a_{n, m}(t, \bar{t}, s) \neq 0\right\} .
$$

In particular, $\operatorname{ord}^{\hbar}(\hbar)=-1, \operatorname{ord}^{\hbar}(\exp (\hbar \partial / \partial s))=0$. The principal symbol (resp. the symbol of order $l$ ) of a difference operator $A=\sum a_{n, m} \hbar^{n} \exp (m \hbar \partial / \partial s)$ is

$$
\begin{gathered}
\sigma^{\hbar}(A) \underset{\text { def }}{=} \hbar^{-\operatorname{ord}^{\hbar}(A)} \sum_{n=\operatorname{ord}^{\hbar}(A)} \sum_{m} a_{n, m} p^{m} \\
\text { (resp. } \left.\sigma_{l}^{\hbar}(A) \underset{\text { def }}{=} \hbar^{-l} \sum_{n=l} \sum_{m} a_{n, m} p^{m}\right) .
\end{gathered}
$$

For example, the condition which we imposed on the coefficients $u_{n}, \bar{u}_{n}$ can be restated $\operatorname{asc}^{\hbar}(L)=\operatorname{ord}^{\hbar}(\bar{L})=0$.

Remark 2.7.2. This "order" coincides with the order of a micro-differential operator if we formally replace $\hbar$ with $\partial_{t_{0}}^{-1}$, where $t_{0}$ is an extra variable (cf. Remark 1.7.2).

The following facts can be easily proved.

Lemma 2.7.3. Let $P_{i}(i=1,2)$ be two difference operators of finite order: $\operatorname{ord}^{\hbar}\left(P_{i}\right)=$ $l_{i}$.

(1) $\operatorname{ord}^{\hbar}\left(P_{1} P_{2}\right)=l_{1}+l_{2}$ and

$$
\sigma^{\hbar}\left(P_{1} P_{2}\right)=\sigma^{\hbar}\left(P_{1}\right) \sigma^{\hbar}\left(P_{2}\right) .
$$


(2) $\operatorname{ord}^{\hbar}\left(\left[P_{1}, P_{2}\right]\right) \leqq l_{1}+l_{2}-1$ and

$$
\sigma_{l_{1}+l_{2}-1}^{\hbar}\left(\left[P_{1}, P_{2}\right]\right)=\hbar\left\{\sigma^{\hbar}\left(P_{1}\right), \sigma^{\hbar}\left(P_{2}\right)\right\}
$$

where $\{$,$\} is the Poisson bracket defined by (2.1.4).$

For example, we have $[\exp (\hbar \partial / \partial s), s]=\hbar \exp (\hbar \partial / \partial s)$, the principal symbol of which gives $\{p, s\}=p$.

Taking the principal symbol of (2.7.1) and using Lemma 2.7.3, we obtain

Proposition 2.7.4. $\left(\mathcal{L}=\sigma^{\hbar}(L), \overline{\mathcal{L}}=\sigma^{\hbar}(\bar{L})\right)$ is a solution of the dispersionless Toda hierarchy (2.1.1), and $\mathcal{B}_{n}=\sigma^{\hbar}\left(B_{n}\right), \overline{\mathcal{B}}_{n}=\sigma^{\hbar}\left(\bar{B}_{n}\right)$.

2.7.2 Dressing operators. Studying the structure of the dressing operators $W, \bar{W}$ of the ordinary Toda lattice hierarchy carefully, we can find counterparts of the dressing functions $\varphi, \bar{\varphi}$, and of the potential $\phi$ of the dispersionless Toda hierarchy.

Proposition 2.7.5. There are dressing operators $W$ and $\bar{W}$

$$
\begin{aligned}
L & =W e^{\hbar \partial / \partial s} W^{-1}, & \bar{L} & =\bar{W} e^{\hbar \partial / \partial s} \bar{W}^{-1} \\
\hbar \frac{\partial W}{\partial t_{n}} W^{-1} & =-\left(W e^{n \hbar \partial / \partial s} W^{-1}\right)_{\leq-1}, & \hbar \frac{\partial \bar{W}}{\partial t_{n}} \bar{W}^{-1} & =\left(W e^{n \hbar \partial / \partial s} W^{-1}\right)_{\geq 0} \\
\hbar \frac{\partial W}{\partial \bar{t}_{n}} W^{-1} & =\left(\bar{W} e^{-n \hbar \partial / \partial s} \bar{W}^{-1}\right)_{\leq-1}, & \hbar \frac{\partial \bar{W}}{\partial \bar{t}_{n}} \bar{W}^{-1} & =-\left(\bar{W} e^{-n \hbar \partial / \partial s} \bar{W}^{-1}\right)_{\geq 0}
\end{aligned}
$$

of the form

$$
\begin{gathered}
W=\exp \left(\hbar^{-1} X(\hbar, t, \bar{t}, s, \exp (\hbar \partial / \partial s))\right) \\
X(\hbar, t, \bar{t}, s, \exp (\hbar \partial / \partial s))=\sum_{n=1}^{\infty} \chi_{n}(\hbar, t, \bar{t}, s) e^{-n \hbar \partial / \partial s}, \\
\operatorname{ord}^{\hbar}(X(\hbar, t, \bar{t}, s, \exp (\hbar \partial / \partial s)))=0, \\
\bar{W}=\exp (\phi(\hbar, t, \bar{t}, s)) \exp \left(\hbar^{-1} \bar{X}(\hbar, t, \bar{t}, s, \exp (\hbar \partial / \partial s))\right), \\
\bar{X}(\hbar, t, \bar{t}, s, \exp (\hbar \partial / \partial s))=\sum_{n=1}^{\infty} \bar{\chi}_{n}(\hbar, t, \bar{t}, s) e^{n \hbar \partial / \partial s}, \\
\operatorname{ord}^{\hbar}(\bar{X}(\hbar, t, \bar{t}, s, \exp (\hbar \partial / \partial s)))=0 .
\end{gathered}
$$


Here $\phi(\hbar, t, \bar{t}, s)$ satisfies $\operatorname{ord}^{\hbar}(\phi) \leqq 0$ and

$$
\begin{gathered}
\frac{\partial \phi}{\partial t_{n}}=\left(L^{n}\right)_{0}, \quad \frac{\partial \phi}{\partial \bar{t}_{n}}=-\left(\bar{L}^{-n}\right)_{0} \\
\frac{1}{\hbar}(\phi(\hbar, t, \bar{t}, s)-\phi(\hbar, t, \bar{t}, s-\hbar))=\log \bar{u}_{0}(\hbar, t, \bar{t}, s)
\end{gathered}
$$

where ( $)_{0}$ is the projection to the multiplication operators (operators not containing $\exp (n \hbar \partial / \partial s), n \neq 0)$.

Conversely, if $W$ and $\bar{W}$ of the form (2.7.10) satisfies (2.7.8) and (2.7.9), then $(L, \bar{L})$ of (2.7.7) gives a soluiton of the Lax equations (2.7.1).

Proof. The proof is mostly parallel to the proof of Proposition 2.1.2 and 2.2.1. First the compatibility conditions of the equations (2.7.11) are a part of the Lax equations and the zero-curvature equations (2.7.1), (2.7.3) (cf. the proof of Proposition 2.1.2). The rest of the proof is analogous to that of Proposition 1.7.5: according to Lemma 2.7.3, the linear space of difference operators of order not greater than $1, \mathcal{E}_{1}=\{P$ : difference operator $\left.\mid \operatorname{ord}^{\hbar}(P) \leqq 1\right\}$, is closed under the commutator and thus is a Lie

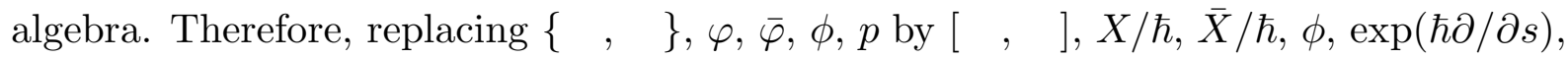
respectively, we can carry over the proof of Proposition 2.2.1 to this case.

As is obvious from this proof, $\varphi, \bar{\varphi}(\S 2.2), \phi(\S 2.1)$ and $W, \bar{W}, \phi$ above are connected as follows (cf. Corollary 1.7.6):

Corollary 2.7.6. Symbols $\sigma^{\hbar}(X)$ and $\sigma^{\hbar}(\bar{X})$ give dressing functions $\varphi, \bar{\varphi}$ of $\mathcal{L}=\sigma^{\hbar}(L)$, $\overline{\mathcal{L}}=\sigma^{\hbar}(\bar{L})$ in the sense of §2.2. Symbol $\sigma^{\hbar}(\phi)$ becomes a corresponding $\phi$-potential defined by (2.1.6). Conversely, if $\varphi$ and $\bar{\varphi}$ are dressing functions of a solution $(\mathcal{L}, \overline{\mathcal{L}})$ of the dispersionless Toda hierarchy and $\phi_{0}$ is the $\phi$-potential, there exist a solution $(L, \bar{L})$ of the Toda lattice hierarchy and dressing operators $W=\exp \left(\hbar^{-1} X\right), \bar{W}=$ $\exp (\phi) \exp \left(\hbar^{-1} \bar{X}\right)$ such that $\sigma^{\hbar}(L)=\mathcal{L}, \sigma^{\hbar}(\bar{L})=\overline{\mathcal{L}}, \sigma^{\hbar}(X)=\varphi, \sigma^{\hbar}(\bar{X})=\bar{\varphi}, \sigma^{\hbar}(\phi)=$ $\phi_{0}$

2.7.3 Orlov operators. The Orlov operators $M$ and $\bar{M}$ of the Toda lattice hierarchy 58 
are defined by

$$
\begin{aligned}
& M=W\left(\sum_{n=1}^{\infty} n t_{n} e^{n \hbar \partial / \partial s}+s\right) W^{-1}=\operatorname{Ad}\left(W \exp \left(\hbar^{-1} t\left(e^{\hbar \partial / \partial s}\right)\right)\right) s \\
& \bar{M}=\bar{W}\left(-\sum_{n=1}^{\infty} n \bar{t}_{n} e^{-n \hbar \partial / \partial s}+s\right) \bar{W}^{-1}=\operatorname{Ad}\left(\bar{W} \exp \left(\hbar^{-1} \bar{t}\left(e^{-\hbar \partial / \partial s}\right)\right)\right) s
\end{aligned}
$$

where $t\left(e^{\hbar \partial / \partial s}\right)=\sum_{n=1}^{\infty} t_{n} e^{n \hbar \partial / \partial s}, \bar{t}\left(e^{-\hbar \partial / \partial s}\right)=\sum_{n=1}^{\infty} \bar{t}_{n} e^{-n \hbar \partial / \partial s}$. These operators are expanded into Laurent series of $L$ and $\bar{L}$ as follows:

$$
\begin{aligned}
& M=\sum_{n=1}^{\infty} n t_{n} L^{n}+s+\sum_{n=1}^{\infty} v_{n}(\hbar, t, \bar{t}, s) L^{-n} \\
& \bar{M}=-\sum_{n=1}^{\infty} n \bar{t}_{n} \bar{L}^{n}+s+\sum_{n=1}^{\infty} \bar{v}_{n}(\hbar, t, \bar{t}, s) \bar{L}^{-n} .
\end{aligned}
$$

It is easy to see the following facts.

\section{Proposition 2.7.7.}

(1) $\operatorname{ord}^{\hbar}(M)=\operatorname{ord}^{\hbar}(\bar{M})=0$;

(2) $[L, M]=\hbar L,[\bar{L}, \bar{M}]=\hbar \bar{L}$;

(3) $M$ and $\bar{M}$ satisfy the Lax equations,

$$
\begin{array}{ll}
\hbar \frac{\partial M}{\partial t_{n}}=\left[B_{n}, M\right], \quad \hbar \frac{\partial M}{\partial \bar{t}_{n}}=\left[\bar{B}_{n}, M\right], \\
\hbar \frac{\partial \bar{M}}{\partial t_{n}}=\left[B_{n}, \bar{M}\right], \quad \hbar \frac{\partial \bar{M}}{\partial \bar{t}_{n}}=\left[\bar{B}_{n}, \bar{M}\right], \quad n=1,2, \ldots
\end{array}
$$

where $B_{n}, \bar{B}_{n}$ are defined by (2.7.3).

Conversely, if $M$ and $\bar{M}$ have the form (2.7.13) and satisfy (1) - (3), then there exist unique dressing operators $W$ and $\bar{W}$ such that $M$ and $\bar{M}$ are expressed as (2.7.12).

We omit the proof since it is essentially the same as those of Proposition 1.3.1, Proposition 1.7.7 and Proposition 2.3.1.

Corollary 2.7.8. $\mathcal{M}=\sigma^{\hbar}(M)$ and $\overline{\mathcal{M}}=\sigma^{\hbar}(\bar{M})$ are the Orlov functions of the dispersionless Toda hierarchy corresponding to $\mathcal{L}=\sigma^{\hbar}(L)$ and $\overline{\mathcal{L}}=\sigma^{\hbar}(\bar{L})$. 
2.7.4 $S$ function and $\tau$ function (free energy). The $S$ functions $S$ and $\bar{S}$ introduced in $§ 2.4$ appear as the phase function of the WKB anaysis of the Toda lattice hierarchy.

We define the Baker-Akhiezer functions

$$
\begin{aligned}
& \Psi=W(\hbar, t, \bar{t}, s) \exp \hbar^{-1}(t(\lambda)+s \log \lambda) \\
& \Psi=\bar{W}(\hbar, t, \bar{t}, s) \exp \hbar^{-1}\left(\bar{t}\left(\bar{\lambda}^{-1}\right)+s \log \bar{\lambda}\right) .
\end{aligned}
$$

They satisfy the linear equations

$$
\begin{array}{llll}
\lambda \Psi=L \Psi, & \hbar \lambda \frac{\partial \Psi}{\partial \lambda}=M \Psi, & \hbar \frac{\partial \Psi}{\partial t_{n}}=B_{n} \Psi, & \hbar \frac{\partial \Psi}{\partial \bar{t}_{n}}=\bar{B}_{n} \Psi \\
\bar{\lambda} \bar{\Psi}=\bar{L} \bar{\Psi}, & \hbar \bar{\lambda} \frac{\partial \bar{\Psi}}{\partial \bar{\lambda}}=\bar{M} \bar{\Psi}, & \hbar \frac{\partial \bar{\Psi}}{\partial t_{n}}=B_{n} \bar{\Psi}, & \hbar \frac{\partial \bar{\Psi}}{\partial \bar{t}_{n}}=\bar{B}_{n} \bar{\Psi} .
\end{array}
$$

Here $\lambda$ and $\bar{\lambda}$ are formal 'spectral parameters' and $t(\lambda)=\sum_{n=1}^{\infty} t_{n} \lambda^{n}, \bar{t}\left(\bar{\lambda}^{-1}\right)=$ $\sum_{n=1}^{\infty} \bar{t}_{n} \bar{\lambda}^{-n}$.

Proposition 2.7.9. Formal power series $\Psi$ and $\bar{\Psi}$ take a WKB asymptotic form as $\hbar \rightarrow 0$ :

$$
\begin{aligned}
& \Psi=\exp \left(\hbar^{-1} S(t, \bar{t}, s, \lambda)+O\left(\hbar^{0}\right)\right), \\
& \bar{\Psi}=\exp \left(\hbar^{-1} \bar{S}(t, \bar{t}, s, \bar{\lambda})+O\left(\hbar^{0}\right)\right),
\end{aligned}
$$

where $S(t, \bar{t}, s, \lambda)$ and $\bar{S}(t, \bar{t}, s, \bar{\lambda})$ are Laurent series of the form

$$
\begin{aligned}
& S(t, \bar{t}, s, \lambda)=t(\lambda)+s \log \lambda+\sum_{n=1}^{\infty} S_{n}(t, \bar{t}, s) \lambda^{-n}, \\
& \bar{S}(t, \bar{t}, s, \lambda)=\bar{t}\left(\bar{\lambda}^{-1}\right)+s \log \bar{\lambda}+\sum_{n=0}^{\infty} \bar{S}_{n}(t, \bar{t}, s) \bar{\lambda}^{n} .
\end{aligned}
$$

In particular, $\bar{S}_{0}(t, \bar{t}, s)=\phi(t, \bar{t}, s)$.

Proof. By the explicit form of the dressing operators $W, \bar{W}(2.7 .10), \Psi$ and $\bar{\Psi}$ have the form

$$
\begin{aligned}
& \Psi=\left(1+O\left(\lambda^{-1}\right)\right) \exp \left(\hbar^{-1} t(\lambda)\right), \\
& \bar{\Psi}=e^{\hbar^{-1} \phi}\left(1+O\left(\bar{\lambda}^{-1}\right)\right) \exp \left(\hbar^{-1} \bar{t}\left(\bar{\lambda}^{-1}\right)\right)
\end{aligned}
$$


Hence by Proposition 2.7.6 (1) and equations (2.7.16) we have the asymptotic form (2.7.19).

Now that $\Psi$ and $\bar{\Psi}$ have the asymptotic form (2.7.18), we can follow the principle of the WKB analysis as in $\$ 1.7 .4$, regarding $S$ and $\bar{S}$ as the phase functions. The Hamilton-Jacobi (eikonal) equations corresponding to (2.7.16) and (2.7.17) are

$$
\begin{aligned}
\lambda & =e^{\partial_{s} S(t, \bar{t}, s, \lambda)}+\sum_{n=0}^{\infty} u_{n+1}^{0}(t, \bar{t}, s) e^{-n \partial_{s} S(t, \bar{t}, s, \lambda)} \\
& =\left.\sigma^{\hbar}(L)\right|_{p=\exp \left(\partial_{s} S(t, \bar{t}, s, \lambda)\right)} \\
d S(t, \bar{t}, s, \lambda) & =\mathcal{M}(\lambda) d \log \lambda+\frac{\partial S}{\partial s} d s+\sum_{n=1}^{\infty} \mathcal{B}_{n}\left(e^{\partial S / \partial s}\right) d t_{n}+\sum_{n=1}^{\infty} \overline{\mathcal{B}}_{n}\left(e^{\partial S / \partial s}\right) d \bar{t}_{n}, \\
\bar{\lambda} & =\left.\sigma^{\hbar}(\bar{L})\right|_{p=\exp \left(\partial_{s} \bar{S}(t, \bar{t}, s, \bar{\lambda})\right)} \\
d \bar{S}(t, \bar{t}, s, \bar{\lambda}) & =\overline{\mathcal{M}}(\bar{\lambda}) d \log \bar{\lambda}+\frac{\partial \bar{S}}{\partial s} d s+\sum_{n=1}^{\infty} \mathcal{B}_{n}\left(e^{\partial \bar{S} / \partial s}\right) d t_{n}+\sum_{n=1}^{\infty} \overline{\mathcal{B}}_{n}\left(e^{\partial \bar{S} / \partial s}\right) d \bar{t}_{n},
\end{aligned}
$$

where

$$
\begin{array}{ll}
\mathcal{M}(\lambda)=\left.\sigma^{\hbar}(M)\right|_{\mathcal{L}=\lambda}, & \mathcal{B}_{n}\left(e^{\partial S / \partial s}\right)=\left.\sigma^{\hbar}\left(B_{n}\right)\right|_{p=\exp (\partial S / \partial s)}, \\
\overline{\mathcal{M}}(\bar{\lambda})=\left.\sigma^{\hbar}(\bar{M})\right|_{\overline{\mathcal{L}}=\bar{\lambda}}, & \overline{\mathcal{B}}_{n}\left(e^{\partial S / \partial s}\right)=\left.\sigma^{\hbar}\left(\bar{B}_{n}\right)\right|_{p=\exp (\partial \bar{S} / \partial s)} .
\end{array}
$$

Thus we arrive at

Proposition 2.7.10. Under the Legendre-type transformation $(t, \bar{t}, s, \lambda) \mapsto(t, \bar{t}, s, p)$ and $(t, \bar{t}, s, \bar{\lambda}) \mapsto(t, \bar{t}, s, p)$ defined by

$$
\exp \frac{\partial S(t, \bar{t}, s, \lambda)}{\partial s}=\exp \frac{\partial \bar{S}(t, \bar{t}, s, \bar{\lambda})}{\partial s}=p
$$

the spectral parameters $\lambda$ and $\bar{\lambda}$ turn into the $\mathcal{L}$ - and $\overline{\mathcal{L}}$-functions of the dispersionless Toda hierarchy,

$$
\lambda=\mathcal{L}(t, \bar{t}, s, p), \quad \bar{\lambda}=\overline{\mathcal{L}}(t, \bar{t}, s, p),
$$

whereas $S(t, \bar{t}, s, \lambda)$ and $\bar{S}(t, \bar{t}, s, \bar{\lambda})$ become the corresponding $S$ - and $\bar{S}$-functions. 
In the presence of $\hbar$, we define the tau function $\tau_{\text {Toda }}=\tau(\hbar, t, \bar{t}, s)$ of the Toda lattice hierarchy as a function that reproduces the Baker-Akhiezer functions as

$$
\begin{aligned}
& \Psi(\hbar, t, \bar{t}, \lambda)=\frac{\tau\left(\hbar, t-\hbar\left[\lambda^{-1}\right], \bar{t}, s\right)}{\tau(\hbar, t, \bar{t}, s)} \exp \hbar^{-1}(t(\lambda)+s \log \lambda) \\
& \bar{\Psi}(\hbar, t, \bar{t}, \bar{\lambda})=\frac{\tau(\hbar, t, \bar{t}-\hbar[\bar{\lambda}], s+\hbar)}{\tau(\hbar, t, \bar{t}, s)} \exp \hbar^{-1}\left(\bar{t}\left(\bar{\lambda}^{-1}\right)+s \log \bar{\lambda}\right)
\end{aligned}
$$

where $\left[\lambda^{-1}\right]$ is defined in (1.7.17).

In the case of $\hbar=1$, this reduces to the ordinary definition. Taking the logarithm of (2.7.23) and comparing them with the WKB asymptotic form (2.7.18) of $\Psi$ and $\bar{\Psi}$, one can easily find that $\log \tau(\hbar, t, \bar{t}, s)$ should behave as

$$
\log \tau(\hbar, t, \bar{t}, s)=\hbar^{-2} F(t, \bar{t}, s)+O\left(\hbar^{-1}\right) \quad(\hbar \rightarrow 0)
$$

with an appropriate scaling function $F(t, \bar{t}, s)$. The leading terms $v_{n}^{0}$ and $\bar{v}_{n}^{0}$ of coefficients $v_{n}$ and $\bar{v}_{n}$ of $M(t, \bar{t}, s, \exp (\hbar \partial / \partial s))$ and $\bar{M}(t, \bar{t}, s, \exp (\hbar \partial / \partial s))$ (see (2.7.13) and Proposition 2.7.7 (1)) can be written as

$$
v_{n}^{0}(t, \bar{t}, s)=\frac{\partial F}{\partial t_{n}}, \quad \bar{v}_{n}^{0}(t, \bar{t}, s)=\frac{\partial F}{\partial \bar{t}_{n}}
$$

and $\bar{S}_{0}=\phi=\partial F / \partial s$ by virtue of $(2.7 .16-19)$. Therefore we can identify $F$ with $\log \tau_{\text {dToda }}$ defined in Proposition 2.4.3.

2.7.5 Riemann-Hilbert problem (twistor construction). The twistor construction of $\S 2.5$ can be extended to the construction of solutions to the ordinary Toda lattice hierarchy in the presence of the parameter $\hbar$.

Proposition 2.7.11. Suppose that

$$
\begin{aligned}
& f\left(\hbar, s, e^{\hbar \partial / \partial s}\right)=\sum_{n \in \mathbb{Z}} f_{n}(\hbar, x) e^{n \hbar \partial / \partial s}, \quad g\left(\hbar, s, e^{\hbar \partial / \partial s}\right)=\sum_{n \in \mathbb{Z}} g_{n}(\hbar, x) e^{n \hbar \partial / \partial s} \\
& \bar{f}\left(\hbar, s, e^{\hbar \partial / \partial s}\right)=\sum_{n \in \mathbb{Z}} \bar{f}_{n}(\hbar, x) e^{n \hbar \partial / \partial s}, \quad \bar{g}\left(\hbar, s, e^{\hbar \partial / \partial s}\right)=\sum_{n \in \mathbb{Z}} \bar{g}_{n}(\hbar, x) e^{n \hbar \partial / \partial s}
\end{aligned}
$$

are difference operators of 0-th order (in the sense of (2.7.5)), $\operatorname{ord}^{\hbar} f=\operatorname{ord}^{\hbar} g=$ $\operatorname{ord}^{\hbar} \bar{f}=\operatorname{ord}^{\hbar} \bar{g}=0$, and that they satisfy the canonical commutation relations $[f, g]=$ 
$\hbar f,[\bar{f}, \bar{g}]=\hbar \bar{f}$. Assume further that difference operators $L, \bar{L}$ of the form (2.7.2) and $M, \bar{M}$ of the form (2.7.13) are given and that $\operatorname{ord}^{\hbar} L=\operatorname{ord}^{\hbar} M=\operatorname{ord}^{\hbar} \bar{L}=\operatorname{ord}^{\hbar} \bar{M}=0$, $[L, M]=\hbar L,[\bar{L}, \bar{M}]=\hbar \bar{L}$.

If

$$
f(\hbar, M, L)=\bar{f}(\hbar, \bar{M}, \bar{L}), \quad g(\hbar, M, L)=\bar{g}(\hbar, \bar{M}, \bar{L})
$$

then $(L, \bar{L})$ gives a solution of the Toda lattice hierarchy, and $(M, \bar{M})$ are the corresponding Orlov operators.

Proof. The assumption on the form of $L, \bar{L}, M, \bar{M}$ implies that there exist difference operators $X, \bar{X}$ and a function $\phi(\hbar, t, \bar{t}, s)$ which are connected with $L, \bar{L}, M, \bar{M}$ through (2.7.7), (2.7.12) and (2.7.10). Following (2.7.10), we denote $W=\exp \left(\hbar^{-1} X\right)$, $\bar{W}=\exp (\phi) \exp \left(\hbar^{-1} \bar{X}\right)$.

Put $P=f(\hbar, M, L)=\bar{f}(\hbar, \bar{M}, \bar{L}), Q=g(\hbar, M, L)=\bar{g}(\hbar, \bar{M}, \bar{L})$ according to $(2.7 .25)$. Differentiating the equation

$$
\begin{aligned}
P & =\operatorname{Ad}\left(W \exp \left(\hbar^{-1} t\left(e^{\hbar \partial / \partial s}\right)\right)\right) f\left(\hbar, s, e^{\hbar \partial / \partial s}\right) \\
& =\operatorname{Ad}\left(\bar{W} \exp \left(\hbar^{-1} \bar{t}\left(e^{-\hbar \partial / \partial s}\right)\right)\right) \bar{f}\left(\hbar, s, e^{\hbar \partial / \partial s}\right)
\end{aligned}
$$

we get

$$
\begin{aligned}
\hbar \frac{\partial P}{\partial t_{n}} & =\left[\hbar \frac{\partial W}{\partial t_{n}} W^{-1}+W e^{n \hbar \partial / \partial s} W^{-1}, P\right] \\
& =\left[\hbar \frac{\partial W}{\partial t_{n}} \bar{W}^{-1}, P\right],
\end{aligned}
$$

and

$$
\begin{aligned}
\hbar \frac{\partial P}{\partial \bar{t}_{n}} & =\left[\hbar \frac{\partial W}{\partial \bar{t}_{n}} W^{-1}, P\right] \\
& =\left[\hbar \frac{\partial \bar{W}}{\partial \bar{t}_{n}} \bar{W}^{-1}+\bar{W} e^{-n \hbar \partial / \partial s} \bar{W}^{-1}, P\right] .
\end{aligned}
$$

Replacing $P$ with $Q$, we obtain the same kind of equations for $Q$. Putting $A=$ $\hbar \frac{\partial W}{\partial t_{n}} W^{-1}+W \exp (n \hbar \partial / \partial s) W^{-1}-\hbar \frac{\partial \bar{W}}{\partial t_{n}} \bar{W}^{-1}$, we can write the equation (2.7.26) and 
the corresponding equation for $Q$ as

$$
[A, P]=[A, Q]=0 \text {. }
$$

The principal symbol of this equation gives

$$
\{\mathcal{A}, \mathcal{P}\}=\{\mathcal{A}, \mathcal{Q}\}=0
$$

where $\mathcal{A}=\sigma^{\hbar}(A), \mathcal{P}=\sigma^{\hbar}(P), \mathcal{Q}=\sigma^{\hbar}(Q)$. Note that $\operatorname{ord}^{\hbar}(A) \leqq 0$, hence the principal symbol of $A$ makes sense. Because of the canonical commutation relation $[f, g]=\hbar f$, we have $[P, Q]=\hbar P$, the principal symbol of which is $\{\mathcal{P}, \mathcal{Q}\}=\mathcal{P}$. Therefore we have $\partial \mathcal{A} / \partial p=\partial \mathcal{A} / \partial s=0$ by (2.7.29) and Lemma 2.6.2. Put $A_{1} \underset{\operatorname{def}}{=} A-\mathcal{A}\left(\operatorname{ord}^{\hbar}\left(A_{1}\right) \leqq-1\right)$. Since $\mathcal{A}$ does not depend on $(p, s)$, it commutes with any difference operator with respect to $s$. Hence the equation (2.7.28) holds if we replace $A$ with $A_{1}$, which again implies $\partial \sigma^{\hbar}\left(A_{1}\right) / \partial p=\partial \sigma^{\hbar}\left(A_{1}\right) / \partial s=0$ by the same argument. Thus we can prove by induction that $A$ does not depend on $s$ and does not contain $\exp (n \hbar \partial / \partial s)$ for any $n(n \neq 0)$.

Again using the form (2.7.10) of $W, \bar{W}$, we have

$$
\begin{aligned}
& \hbar \frac{\partial W}{\partial t_{n}} W^{-1}=-\left(W e^{n \hbar \partial / \partial s} W^{-1}\right)_{\leq-1}, \\
& \hbar \frac{\partial \bar{W}}{\partial t_{n}} \bar{W}^{-1}=\left(W e^{n \hbar \partial / \partial s} W^{-1}\right)_{\geq 0}+A .
\end{aligned}
$$

Equation (2.7.30) leads to the Lax equations for $L$ and $M$ with respect to $t_{n}$, the first equations of (2.7.1) and (2.7.14), directly. Since $A$ commutes with any difference operator, equation (2.7.31) implies the Lax equations for $\bar{L}$ and $\bar{M}$ with respect to $t_{n}$, the third equations of (2.7.1) and (2.7.14).

The other Lax equations in (2.7.1) and (2.7.14) are proved similarly by (2.7.27). From the last statement of Proposition 2.7.7, $M$ and $\bar{M}$ are Orlov operators corresponding to $L$ and $\bar{L}$. (Note that $W$ is one of the dressing operators corresponding to $(L, M)$, but, $\bar{W}$ is not a dressing operator in general because of the remainder $A$ in (2.7.31).)

Let us call the quadruplet $(f, g, \bar{f}, \bar{g})$ above the twistor data of the Toda lattice hierarchy. 
Proposition 2.7.12. Any solution of the Toda lattice hierarchy possesses a twistor data, i.e., if $(L, M, \bar{L}, \bar{M})$ is a soluiton of the Toda lattice hierarchy in the presence of $\hbar$, there exists a quadruplet $(f, g, \bar{f}, \bar{g})$ which satisfies the canonical commutation relation $[f, g]=\hbar f,[\bar{f}, \bar{g}]=\hbar \bar{f}$ and (2.7.25).

Proof. Let $W$ and $\bar{W}$ be the dressing operators chosen in Proposition 2.7.5. We can prove by the same method as Propositio 1.7.8 that twistor data are given by

$$
\begin{aligned}
& f\left(\hbar, s, e^{\hbar \partial / \partial s}\right)=W_{0}^{-1} e^{\hbar \partial / \partial s} W_{0}, \quad g\left(\hbar, s, e^{\hbar \partial / \partial s}\right)=W_{0}^{-1} s W_{0}, \\
& \bar{f}\left(\hbar, s, e^{\hbar \partial / \partial s}\right)=\bar{W}_{0}^{-1} e^{\hbar \partial / \partial s} \bar{W}_{0}, \quad \bar{g}\left(\hbar, s, e^{\hbar \partial / \partial s}\right)=\bar{W}_{0}^{-1} s \bar{W}_{0},
\end{aligned}
$$

where $W_{0}=W(t=0, \bar{t}=0), \bar{W}_{0}=\bar{W}(t=0, \bar{t}=0)$.

The principal symbols $\sigma^{\hbar}(f), \sigma^{\hbar}(g), \sigma^{\hbar}(\bar{f}), \sigma^{\hbar}(\bar{g})$ give the twistor data for the solution $\left(\mathcal{L}=\sigma^{\hbar}(L), \mathcal{M}=\sigma^{\hbar}(M), \overline{\mathcal{L}}=\sigma^{\hbar}(\bar{L}), \overline{\mathcal{M}}=\sigma^{\hbar}(\bar{M})\right)$ of the dispersionless Toda hierarchy.

The solution to (2.7.25) is unique in the following infinitesimal sense.

Proposition 2.7.13. Suppose $(L(\varepsilon), M(\varepsilon), \bar{L}(\varepsilon), \bar{M}(\varepsilon))$ is a one-parameter family of solutions to (2.7.25) that are regular in a neighborhood of $\varepsilon=0$. Then $(L(\varepsilon), M(\varepsilon), \bar{L}(\varepsilon), \bar{M}(\varepsilon))=$ $(L(0), M(0), \bar{L}(0), \bar{M}(0))$.

We omit the proof since it is essentially the same as that of Proposition 1.7.13.

2.7.6. $W_{1+\infty}$ symmetry. Here we analyze the quasi-classical limit of the $W_{1+\infty}$ symmetry of the Toda lattice hierarchy. As in the case of the KP hierarchy, there are two ways to introduce this symmetry: to deform the twistor data, and to use the group action on the parameter space of the solutions.

As in $\S 2.6$, we consider the deformation of the twistor data $(f, g, \bar{f}, \bar{g})$ of the form

$$
\begin{aligned}
& (f, g) \mapsto(f, g) \circ \operatorname{Ad} \exp (-\varepsilon Y), \\
& (\bar{f}, \bar{g}) \mapsto(\bar{f}, \bar{g}) \circ \operatorname{Ad} \exp (-\varepsilon \bar{Y}),
\end{aligned}
$$

where $Y$ and $\bar{Y}$ are difference operators of order not greater than $1, \operatorname{ord}^{\hbar}(Y) \leqq 1$, $\operatorname{ord}^{\hbar}(\bar{Y}) \leqq 1$. 
Proposition 2.7.14. i) The above symmetry of the Lax operators are given explicitly by

$$
\begin{aligned}
\delta_{Y, \bar{Y}} L & =\left[Y(\hbar, M, L)_{\leq-1}-\bar{Y}(\hbar, \bar{M}, \bar{L})_{\leq-1}, L\right], \\
\delta_{Y, \bar{Y}} M & =\left[Y(\hbar, M, L)_{\leq-1}-\bar{Y}(\hbar, \bar{M}, \bar{L})_{\leq-1}, M\right], \\
\delta_{Y, \bar{Y}} \bar{L} & =\left[\bar{Y}(\hbar, \bar{M}, \bar{L})_{\geq 0}-Y(\hbar, M, L)_{\geq 0}, \bar{L}\right], \\
\delta_{Y, \bar{Y}} \bar{M} & =\left[\bar{Y}(\hbar, \bar{M}, \bar{L})_{\geq 0}-Y(\hbar, M, L)_{\geq 0}, \bar{M}\right] .
\end{aligned}
$$

ii) The symmetries of the dressing operators are given by

$$
\begin{aligned}
& \delta_{Y, \bar{Y}} W \cdot W^{-1}=Y(\hbar, M, L)_{\leq-1}-\bar{Y}(\hbar, \bar{M}, \bar{L})_{\leq-1}, \\
& \delta_{Y, \bar{Y}} \bar{W} \cdot \bar{W}^{-1}=\bar{Y}(\hbar, \bar{M}, \bar{L})_{\geq 0}-Y(\hbar, M, L)_{\geq 0} .
\end{aligned}
$$

Symmetries of $X$ and $\bar{X}$ (2.7.10) are induced by these formulae. A symmetry of the potential $\phi$ (2.7.11) consistent with (2.7.33) is given by

$$
\delta_{Y, \bar{Y}} \phi=\bar{Y}(\hbar, \bar{M}, \bar{L})_{0}-Y(\hbar, M, L)_{0} .
$$

Proof. The proof is the same as that of Proposition 2.6.1 (see also the proof of Proposition 2.7.11). For example, (2.7.34) comes from the coefficients of $\varepsilon^{1}$ of the equation

$$
\begin{aligned}
W_{\varepsilon} e^{t(\exp (\hbar \partial / \partial s))} e^{-\varepsilon Y} e^{-t(\exp (\hbar \partial / \partial s))} W^{-1} & \\
& =a_{\varepsilon} \bar{W}_{\varepsilon} e^{\bar{t}(\exp (-\hbar \partial / \partial s))} e^{-\varepsilon \bar{Y}} e^{-\bar{t}(\exp (-\hbar \partial / \partial s))} \bar{W}^{-1},
\end{aligned}
$$

where $W_{\varepsilon}$ and $\bar{W}_{\varepsilon}$ are deformed dressing operators and $a_{\varepsilon}$ is a scalar. The statement i) immediately follows from ii).

Taking the principal symbol of these expressions, we obtain the $w_{1+\infty}$ symmetry of the dispersionless Toda hierarchy in Proposition 2.6.1.

In the earlier paper [4] we studied these symmetries in terms of vertex operators and free fermions [15]:

$$
\begin{aligned}
Z(\hbar, \tilde{\lambda}, \lambda) & =\frac{\exp \left(\hbar^{-1}[t(\tilde{\lambda})-t(\lambda)]\right)(\tilde{\lambda} / \lambda)^{s / \hbar} \exp \left(\hbar\left[-\tilde{\partial}_{t}\left(\tilde{\lambda}^{-1}\right)+\tilde{\partial}_{t}\left(\lambda^{-1}\right)\right]\right)-1}{\tilde{\lambda}-\lambda} \\
\bar{Z}(\hbar, \tilde{\lambda}, \lambda) & =\frac{\exp \left(\hbar^{-1}\left[\bar{t}\left(\tilde{\lambda}^{-1}\right)-\bar{t}\left(\lambda^{-1}\right)\right]\right)(\tilde{\lambda} / \lambda)^{s / \hbar} \exp \left(\hbar\left[-\tilde{\partial}_{\bar{t}}(\tilde{\lambda})+\tilde{\partial}_{\bar{t}}(\lambda)\right]\right)-1}{\tilde{\lambda}^{-1}-\lambda^{-1}}
\end{aligned}
$$


where

$$
\tilde{\partial}_{t}\left(\lambda^{-1}\right)=\sum_{n=1}^{\infty} \frac{\lambda^{-n}}{n} \frac{\partial}{\partial t_{n}}, \quad \tilde{\partial}_{\bar{t}}(\lambda)=\sum_{n=1}^{\infty} \frac{\lambda^{n}}{n} \frac{\partial}{\partial \bar{t}_{n}} .
$$

The generators of $W_{1+\infty}$ symmetries appear as the coefficients $W_{n}^{(\ell)}(\hbar)$ and $\bar{W}_{n}^{(\ell)}(\hbar)$ of the expansion

$$
\begin{gathered}
Z(\hbar, \tilde{\lambda}, \lambda)=\sum_{\ell=1}^{\infty} \frac{(\tilde{\lambda}-\lambda)^{\ell-1}}{(\ell-1) !} W^{(\ell)}(\hbar, \lambda), \\
W^{(\ell)}(\hbar, \lambda)=\sum_{n=-\infty}^{\infty} W_{n}^{(\ell)}(\hbar) \lambda^{-n-\ell},
\end{gathered}
$$

( $n \in \mathbb{Z}, \ell \geqq 1$ ) and the same kind of expansion of $\bar{Z}$. These symmetry generators are differential operators of finite order in $t$ and $\bar{t}$ respectively, and differ from the ordinary $(\hbar=1)$ definition [15] by the simple rescaling

$$
\begin{aligned}
& t_{n} \rightarrow \hbar^{-1} t_{n}, \quad \frac{\partial}{\partial t_{n}} \rightarrow \hbar \frac{\partial}{\partial t_{n}} \\
& \bar{t}_{n} \rightarrow \hbar^{-1} \bar{t}_{n}, \quad \frac{\partial}{\partial \bar{t}_{n}} \rightarrow \hbar \frac{\partial}{\partial \bar{t}_{n}} .
\end{aligned}
$$

We have thus essentially the same $W_{1+\infty}$ symmetries as the KP hierarchy, but now in duplicate.

As is proved in [4], the $w_{1+\infty}$ symmetries in $\S 2.6$ are reproduced as quasi-classical limit of the above $W_{1+\infty}$ symmetries by

$$
w_{n}^{(\ell)} \log \tau_{\text {dToda }}=\lim _{\hbar \rightarrow 0} \tau_{\text {Toda }}(\hbar, t, \bar{t}, s)^{-1} \hbar^{\ell} W_{n}^{(\ell)}(\hbar) \tau_{\text {Toda }}(\hbar, t, \bar{t}, s)
$$

where

$$
w_{n}^{(\ell)} \log \tau_{\text {dToda }}=\operatorname{Res} \frac{1}{\ell} \mathcal{M}^{\ell} \mathcal{L}^{n} d_{p} \log \mathcal{L}
$$

which corresponds to (2.6.17) with $F=-s^{\ell-1} p^{n}, \bar{F}=0$. The other half $\bar{w}_{n}^{(\ell)}(F=0$, $\left.\bar{F}=-s^{\ell-1} p^{n}\right)$ comes from $\bar{W}_{n}^{(\ell)}$ in the same way.

In the ferminonic language, we can unify these two expressions of symmetries, Proposition 2.7.14 and (2.7.42). (We use the same notations as in §1.7.6.) As is well known ([40], [41]), when $\hbar=1$, the generic tau function is given by the expression

$$
\tau(t, \bar{t}, s)=\left\langle s\left|e^{H(t)} g_{-} g_{0} g_{+} e^{-\bar{H}(\bar{t})}\right| s\right\rangle
$$


where $H(z)$ and $\bar{H}(\bar{t})$ are generators of time evolutions,

$$
\begin{aligned}
& H(t)=\sum_{n=1}^{\infty} t_{n} H_{n}, \quad \bar{H}(\bar{t})=\sum_{n=1}^{\infty} \bar{t}_{n} H_{-n}, \\
& H_{n}=\sum_{i=-\infty}^{\infty}: \psi_{i} \psi_{i+n}^{*}:
\end{aligned}
$$

(cf. (1.7.42)), and $g_{+}, g_{-}$and $g_{0}$ are Clifford operators belonging to the upper triangular, the lower triangular and the diagonal part of $\mathfrak{g l}(\infty)$ respectively.

In our context, these Clifford operators have the following form.

Proposition 2.7.15. The tau function with well-defined quasi-classical limit in our sense is expressed as

$$
\tau(\hbar, t, \bar{t}, s)=\left\langle\hbar^{-1} s\left|e^{H(t) / \hbar} g_{-}(\hbar) g_{0}(\hbar) g_{+}(\hbar) e^{-\bar{H}(\bar{t}) / \hbar}\right| \hbar^{-1} s\right\rangle
$$

where $g_{ \pm, 0}(\hbar)$ are Clifford operators of the form

$$
\begin{aligned}
& g_{-}(\hbar)=\exp \left(-\hbar^{-1} \mathcal{O}_{X}(\hbar)\right), \\
& g_{+}(\hbar)=\exp \left(\hbar^{-1} \mathcal{O}_{\bar{X}}(\hbar)\right), \\
& g_{0}(\hbar)=\exp \left(\hbar^{-1} \mathcal{O}_{\phi}(\hbar)\right) .
\end{aligned}
$$

Here $\mathcal{O}_{X}, \mathcal{O}_{\bar{X}}, \mathcal{O}_{\phi}$ are determined from the dressing operators $X, \bar{X}$ and $\phi$ (2.7.10) as follows (see [41]): Suppose $X$ is expanded as

$$
X\left(\hbar, t=0, \bar{t}=0, s, e^{\hbar \partial / \partial s}\right)=\sum_{n=1}^{\infty} x_{n, \ell}(\hbar) s^{\ell-1} e^{(n+\ell-1) \hbar \partial / \partial s}
$$

Then $\mathcal{O}_{X}$ is given by

$$
\begin{gathered}
\mathcal{O}_{X}=\oint: A\left(\lambda, \hbar \frac{\partial}{\partial \lambda}\right) \psi(\lambda) \cdot \psi^{*}(\lambda): \frac{d \lambda}{2 \pi i}, \\
A\left(\lambda, \hbar \frac{\partial}{\partial \lambda}\right)=\sum_{n=1}^{\infty} x_{n, \ell}(\hbar) \lambda^{n+\ell-1}\left(\hbar \frac{\partial}{\partial \lambda}\right)^{\ell-1},
\end{gathered}
$$

where $A \psi(\lambda) \cdot \psi^{*}(\lambda)$ is understood to be "A $\psi(\lambda)$ times $\psi^{*}(\lambda)$ ". Other Clifford operators $\mathcal{O}_{\bar{X}}$ and $\mathcal{O}_{\phi}$ are determined in the same manner. 
Proof. The algebra generated by the multiplication operator $s$ and the difference operator $\exp (\hbar \partial / \partial s)$ has a representation in the algebra of $\mathbb{Z} \times \mathbb{Z}$-matrices, $\mathfrak{g l}(\infty)$, as

$$
\begin{aligned}
s & \leftrightarrow\left((\nu+1) \hbar \delta_{\mu-1, \nu}\right)_{\mu, \nu \in \mathbb{Z},} \\
e^{\hbar \partial / \partial s} & \leftrightarrow\left(\delta_{\mu+1, \nu}\right)_{\mu, \nu \in \mathbb{Z},} \\
s^{\ell-1} e^{(n+\ell-1) \hbar \partial / \partial s} & \leftrightarrow\left((\nu-n)(\nu-n-1) \cdots(\nu-n-\ell+2) \hbar^{\ell-1} \delta_{\mu+n, \nu}\right)_{\mu, \nu \in \mathbb{Z} \cdot}
\end{aligned}
$$

(This gives a representation of the algebra of difference operators on the space of functions on the lattice $\{s=\hbar n\}_{n \in \mathbb{Z}}$.) Therefore, through the identification of : $\psi_{m} \psi_{n}^{*}:$ with the matrix unit $E_{m, n}$, the difference operator $X(\hbar, t=0, \bar{t}=0, s, \exp (\hbar \partial / \partial s))$ can be identified with $\mathcal{O}_{X}(\hbar)$ in (2.7.48). Similarly we obtain the Clifford operator expressions $\mathcal{O}_{\bar{X}}$ and $\mathcal{O}_{\phi}$ in $(2.7 .46)$ for $\bar{X}$ and $\phi$. According to the general theory on the relation of the initial value of the dressing operators with the tau function (e.g., [42], [41]), the tau function has the form (2.7.45).

The action of $Z(\hbar, \tilde{\lambda}, \lambda)$ and $\bar{Z}(\hbar, \tilde{\lambda}, \lambda)$ is realized by insertion of a fermion bilinear form:

$$
\begin{aligned}
& Z(\hbar, \tilde{\lambda}, \lambda) \tau(\hbar, t, \bar{t}, s)=\left\langle\hbar^{-1} s\left|e^{H(t) / \hbar}: \psi(\tilde{\lambda}) \psi^{*}(\lambda): g(\hbar) e^{-\bar{H}(\bar{t}) / \hbar}\right| \hbar^{-1} s\right\rangle \\
& \bar{Z}(\hbar, \tilde{\lambda}, \lambda) \tau(\hbar, t, \bar{t}, s)=\left\langle\hbar^{-1} s\left|e^{H(t) / \hbar} g(\hbar): \psi(\tilde{\lambda}) \psi^{*}(\lambda): e^{-\bar{H}(\bar{t}) / \hbar}\right| \hbar^{-1} s\right\rangle,
\end{aligned}
$$

where $g(\hbar)=g_{-}(\hbar) g_{0}(\hbar) g_{+}(\hbar)$. In particular,

$$
\begin{aligned}
& W_{n}^{(\ell)}(\hbar) \tau(\hbar, t, \bar{t}, s)=\left\langle\hbar^{-1} s\left|e^{H(t) / \hbar} \mathcal{O}_{k}^{(\ell)}(\hbar) g(\hbar) e^{-\bar{H}(\bar{t}) / \hbar}\right| \hbar^{-1} s\right\rangle, \\
& \bar{W}_{n}^{(\ell)}(\hbar) \tau(\hbar, t, \bar{t}, s)=\left\langle\hbar^{-1} s\left|e^{H(t) / \hbar} g(\hbar) \mathcal{O}_{k}^{(\ell)}(\hbar) e^{-\bar{H}(\bar{t}) / \hbar}\right| \hbar^{-1} s\right\rangle
\end{aligned}
$$

where $\mathcal{O}_{k}^{(\ell)}(\hbar)$ is defined by $(1.7 .51)$.

Proposition 2.7.16. The symmetry of the tau function by $W_{n}^{(\ell)}$ and $\bar{W}_{n}^{(\ell)}$ induces the symmetries of $L, M, \bar{L}, \bar{M}, W, \bar{W}$ of the form (2.7.33), (2.7.34) for $(Y, \bar{Y})=$ $\left(-s^{\ell-1} e^{(n+\ell-1) \hbar \partial / \partial s}, 0\right)$ and $(Y, \bar{Y})=\left(0,-s^{\ell-1} e^{(n+\ell-1) \hbar \partial / \partial s}\right)$, respectively.

Proof. We prove the proposition only for $W_{n}^{(\ell)}$. The equation (2.7.36) for $(Y, \bar{Y})=$ $\left(-s^{\ell-1} e^{(n+\ell-1) \hbar \partial / \partial s}, 0\right)$ at $t=\bar{t}=0$ becomes

$$
W_{\varepsilon, 0} e^{-\varepsilon Y(\hbar, s, \exp (\hbar \partial / \partial s))} W_{0}^{-1}=a_{\varepsilon} \bar{W}_{\varepsilon, 0} \bar{W}_{0}^{-1}
$$


Here the index 0 of $W_{0}$ etc. means the value at $t=\bar{t}=0$. Since a central factor changes the tau function only up to a scalar factor, it does not change $L, M$ etc. Hence we neglect this central correction $a_{\varepsilon}$ below. The fermion bilinear form $\mathcal{O}_{k}^{(\ell)}$ corresponds to $-Y=s^{\ell-1} e^{(n+\ell-1) \hbar \partial / \partial s}$ through (2.7.49) (up to a central factor). Hence (2.7.52) corresponds to the expression

$$
g_{-, \varepsilon}(\hbar)^{-1} e^{\varepsilon \mathcal{O}_{k}^{(\ell)}} g_{-}(\varepsilon)=g_{0, \varepsilon}(\hbar) g_{+, \varepsilon}(\hbar) g_{+}(\hbar)^{-1} g_{0}(\hbar)^{-1},
$$

where $g_{ \pm, \varepsilon}, g_{0, \varepsilon}$ denote the deformed Clifford operators. Therefore, taking this equation into account, we obtain from (2.7.45)

$$
\begin{aligned}
\tau_{\varepsilon}(\hbar, t, \bar{t}, s) & =\left\langle\hbar^{-1} s\left|e^{H(t) / \hbar} g_{-, \varepsilon}(\hbar) g_{0, \varepsilon}(\hbar) g_{+, \varepsilon}(\hbar) e^{-\bar{H}(\bar{t}) / \hbar}\right| \hbar^{-1} s\right\rangle \\
& =\left\langle\hbar^{-1} s\left|e^{H(t) / \hbar} e^{\varepsilon \mathcal{O}_{k}^{(\ell)}} g_{-}(\hbar) g_{0}(\hbar) g_{+}(\hbar) e^{-\bar{H}(\bar{t}) / \hbar}\right| \hbar^{-1} s\right\rangle .
\end{aligned}
$$

This proves the assertion of the proposition.

The bosonic and fermionic representations are thus connected, and the intrinsic reason of the coincidence of quasi-classical limit of the symmetries (2.7.41) with the symmetries defined by the deformation of the twistor data in $\S 2.6$ becomes clear.

For the moment, the bosonic language looks more preferable, because the fermionic representation is valid only for discrete values $s \in \hbar^{-1} \mathbb{Z}$.

\subsection{Relation with the dispersionless KP hierarchy.}

We can lift up the solution of the dispersionless Toda hierarchy to the ordinary Toda lattice hierarchy (Corollary 2.7.6). A solution of the Toda lattice hierarchy gives a solution of the KP hierarchy if the variable $s$ and $\bar{t}$ are considered to be parameters ([21]). A solution of the dispersionless Toda hierarchy should therefore give a solution of the dispersionless KP hierarchy when $s$ and $\bar{t}$ are fixed.

This can be verified directly as follows.

Proposition 2.8.1. Let $(\mathcal{L}, \overline{\mathcal{L}})$ be a solution of the dispersionless Toda hierarchy (2.1.1). Then $\mathcal{L}(t, \bar{t}, s)$ is a solution of the dispersionless KP hierarchy (1.1.1) when 
we substitute $t_{1}+x$ to $t_{1}$ and identify $\mathcal{B}_{1}$ with $k$ :

$$
\left.\frac{\partial \mathcal{L}}{\partial t_{n}}\right|_{k \text { fixed }}=\left\{\mathcal{B}_{n}, \mathcal{L}\right\}_{\mathrm{KP}}
$$

Here

$$
\{f, g\}_{\mathrm{KP}}=\frac{\partial f}{\partial k} \frac{\partial g}{\partial x}-\frac{\partial f}{\partial x} \frac{\partial g}{\partial k}
$$

as in $\S 1.1$ (1.1.2). Note that the projection onto a polynomial in $p$ is equal to the projection onto a polynomial in $k$, since $k=\mathcal{B}_{1}=p+u_{1}(t, \bar{t}, s)$. Thus $\mathcal{B}_{n}=\left(\mathcal{L}^{n}\right)_{\geq 0}$ in the sense of (1.1.1) as well as in the sense of (2.1.1).

Proof. Because of the Lax equations (2.1.1) we have

$$
\begin{aligned}
\left.\frac{\partial \mathcal{L}}{\partial t_{n}}\right|_{k \text { fixed }} & =\frac{\partial \mathcal{L}}{\partial t_{n}}-\frac{\partial \mathcal{L}}{\partial k} \frac{\partial \mathcal{B}_{1}}{\partial t_{n}} \\
& =p \frac{\partial \mathcal{B}_{n}}{\partial p} \frac{\partial \mathcal{L}}{\partial s}-p \frac{\partial \mathcal{B}_{n}}{\partial s} \frac{\partial \mathcal{L}}{\partial p}-\frac{\partial \mathcal{L}}{\partial k} \frac{\partial \mathcal{B}_{1}}{\partial t_{n}}
\end{aligned}
$$

In particular, (2.8.1) $n=1$ implies

$$
p \frac{\partial \mathcal{L}}{\partial s}=\left.\frac{\partial \mathcal{L}}{\partial t_{1}}\right|_{k \text { fixed }}+\frac{\partial \mathcal{L}}{\partial k} \frac{\partial \mathcal{B}_{1}}{\partial t_{1}}+p \frac{\partial \mathcal{B}_{1}}{\partial s} \frac{\partial \mathcal{L}}{\partial p}
$$

Substituting this into (2.8.1) and using $\partial / \partial p=\partial / \partial k$, we obtain

$$
\left.\frac{\partial \mathcal{L}}{\partial t_{n}}\right|_{k \text { fixed }}=\left.\frac{\partial \mathcal{B}_{n}}{\partial k} \frac{\partial \mathcal{L}}{\partial t_{1}}\right|_{k \text { fixed }}-\left(\frac{\partial \mathcal{B}_{1}}{\partial t_{n}}+\left\{\mathcal{B}_{1}, \mathcal{B}_{n}\right\}-\frac{\partial \mathcal{B}_{n}}{\partial k} \frac{\partial \mathcal{B}_{1}}{\partial t_{1}}\right) \frac{\partial \mathcal{L}}{\partial k} .
$$

By the zero-curvature equation (2.1.5), the right hand side of (2.8.3) is

$$
\left.\frac{\partial \mathcal{B}_{n}}{\partial k} \frac{\partial \mathcal{L}}{\partial t_{1}}\right|_{k \text { fixed }}-\left.\frac{\partial \mathcal{B}_{n}}{\partial t_{1}}\right|_{k \text { fixed }} \frac{\partial \mathcal{L}}{\partial k}=\left\{\mathcal{B}_{n}, \mathcal{L}\right\}_{\mathrm{KP}}
$$

which proves the proposition.

In the gauge chosen in Lemma 2.1.3, another solution of the dispersionless KP hierarchy arises from $\mathcal{L}^{\prime}\left(t, s\right.$ fixed), when we identify $k=\mathcal{B}_{1}^{\prime}$. 


\section{Appendix A. Several faCts on Differential Lie algebras}

Here we collect several facts on formal properties of differential Lie algebras. Proofs are omitted.

Let $\mathfrak{g}$ be a Lie algebra with a derivation $\partial: \mathfrak{g} \rightarrow \mathfrak{g}$. Actually, we have in mind such examples as the Lie algebra of Laurent series (Lie bracket $=$ Poisson bracket, derivation $=$ differentiation with respect to parameters $\left.t_{n} ; \S 1.2, \S 2.2\right)$ or the Lie algebra of microdifferential operators or difference operators $($ Lie bracket $=$ commutator, derivation $=$ differentiation with respect to parameters $\left.t_{n} ; \S 1.7, \S 2.7\right)$.

In this section we always assume existence of suitable topology of $\mathfrak{g}$ in which each series under consideration converges.

Lemma A.1. For all $X, Y \in \mathfrak{g}$,

$$
\partial\left(e^{\operatorname{ad} Y} X\right)=e^{\operatorname{ad} Y}(\partial X)+\left[\nabla_{Y} Y, e^{\operatorname{ad} Y} X\right]
$$

where

$$
\nabla_{Z} W=\sum_{n=0}^{\infty} \frac{1}{(n+1) !}(\operatorname{ad} Z)^{n}(\partial W)=\frac{e^{\operatorname{ad} Z}-1}{\operatorname{ad} Z}(\partial W) .
$$

By the Hausdorff-Campbell formula, there exists a unique series (the Hausdorff series) $H(X, Y) \in \mathfrak{g}$ of $X, Y \in \mathfrak{g}$ such that

$$
\exp (H(X, Y))=\exp (X) \exp (Y)
$$

Lemma A.2. For all $X, Y \in \mathfrak{g}$,

$$
\nabla_{H(X, Y)} H(X, Y)=\nabla_{X} X+e^{\operatorname{ad} X} \nabla_{Y} Y
$$

To consider zero-curvature representation of integrable systems, we also need the following Lemma for a differential Lie algebra $\mathfrak{g}$ with two derivations $\partial_{1}, \partial_{2}$.

Lemma A.3. For $X, Y, Z \in \mathfrak{g}$ define

$$
\tilde{X}=e^{\operatorname{ad} Z} X+\nabla_{1, Z} Z, \quad \tilde{Y}=e^{\operatorname{ad} Z} Y+\nabla_{2, Z} Z,
$$


where $\nabla_{1, Z}$ and $\nabla_{2, Z}$ are defined by (A.1) for $\partial=\partial_{1}$ and $\partial=\partial_{2}$. Then the following two statements are equivalent.

(1) $\left[\partial_{1}-X, \partial_{2}-Y\right]=0$, i.e., $\partial_{2} X-\partial_{1} Y+[X, Y]=0$.

(2) $\left[\partial_{1}-\tilde{X}, \partial_{2}-\tilde{Y}\right]=0$, i.e., $\partial_{2} \tilde{X}-\partial_{1} \tilde{Y}+[\tilde{X}, \tilde{Y}]=0$.

\section{Appendix B. Quasi-Classical limit of Hirota equations of KP hierarchy}

Here we show that some of the Hirota equations have quasi-classical limit, and propose a conjecture that these equations characterize the tau function (free energy) of the dispersionless KP hierarchy.

It is well-known that the tau function of the KP hierarchy is characterized by the Hirota equations, a generating function of which is

$$
\sum_{j=0}^{\infty} p_{j}\left(-2 \hbar^{-1} y\right) p_{j+1}\left(\hbar \tilde{D}_{y}\right) \exp \left(\sum_{l=1}^{\infty} y_{l} D_{t_{l}}\right) \tau(t) \cdot \tau(t)=0
$$

Here $y=\left(y_{1}, y_{2}, \ldots\right)$ is a series of parameters, $p_{j}(y)$ are Schur polynomials defined by

$$
\exp \left(\sum_{l=1}^{\infty} y_{l} \lambda^{l}\right)=\sum_{j=0}^{\infty} p_{j}(y) \lambda^{j}
$$

$D$ is the Hirota operator defined by $D_{x} f(x) \cdot g(x)=\left.\partial_{y} f(x+y) g(x-y)\right|_{y=0}$ and $\tilde{\partial}=$ $\left(\partial / 1, \partial^{2} / 2, \partial^{3} / 3, \ldots\right)$. (We have introduced the Planck constant $\hbar$ as in $\left.\S 1.7.\right)$ Expanded into monomials of $y$, (B.1) gives the Plücker relations for the Plücker embedding (1.7.35) when $\hbar=1$.

These equations, however, do not possess a good quasi-classical limit in general. In fact, if we insert the prescribed asymptotic behaviour of the tau function, $\log \tau(\hbar, t)=$ $\hbar^{-2} F(t)+O\left(\hbar^{-1}\right)(\hbar \rightarrow 0)$, the $\hbar$-expansion of (B.1) becomes extremely complicated and gives rise to no useful information.

Nevertheless, there are a series of Hirota equations which do have quasi-classical limit: the differential Fay identity. 
Proposition B.1. (Differential Fay identity)

$$
\begin{aligned}
& \hbar \partial_{t_{1}} \tau\left(t-\hbar\left[\mu^{-1}\right]\right) \tau\left(t-\hbar\left[\lambda^{-1}\right]\right)-\tau\left(t-\hbar\left[\mu^{-1}\right]\right) \hbar \partial_{t_{1}} \tau\left(t-\hbar\left[\lambda^{-1}\right]\right) \\
& -(\lambda-\mu) \tau\left(t-\hbar\left[\mu^{-1}\right]\right) \tau\left(t-\hbar\left[\lambda^{-1}\right]\right)+(\lambda-\mu) \tau(t) \tau\left(t-\hbar\left[\mu^{-1}\right]-\hbar\left[\lambda^{-1}\right]\right)=0 .
\end{aligned}
$$

Here $\mu$ and $\lambda$ are free parameters and $\left[\lambda^{-1}\right]=\left(\lambda^{-1}, \lambda^{-2} / 2, \lambda^{-3} / 3, \ldots\right)$.

See [37] for the proof. The coefficient of $\mu^{-m} \lambda^{-n}$ gives the bilinear equation

$$
\begin{aligned}
\chi_{0, m+1}(-\hbar \tilde{\partial}) \tau(t) \chi_{1, n+1}(-\hbar \tilde{\partial}) \tau(t)- & \chi_{1, m+1}(-\hbar \tilde{\partial}) \tau(t) \chi_{0, n+1}(-\hbar \tilde{\partial}) \tau(t) \\
& +\chi_{n+1, m+1}(-\hbar \tilde{\partial}) \tau(t) \chi_{0,1}(-\hbar \tilde{\partial}) \tau(t)=0,
\end{aligned}
$$

where $\chi_{k, l}$ is the Schur function corresponding to the partition $(k, l-1)$. Thus (B.2) is a part of the Plücker relations. From this equation we can derive a set of equations for the free energy $\log \tau_{\mathrm{dKP}}$ of the dispersionless KP hierarchy.

Proposition B.2. The tau function (or free energy) of the dispersionless KP hierarchy satisfies the equation

$$
\sum_{m, n=1}^{\infty} \mu^{-m} \lambda^{-n} \frac{1}{m n} \frac{\partial^{2}}{\partial t_{m} \partial t_{n}} \log \tau_{\mathrm{dKP}}=\log \left(1+\sum_{n=1}^{\infty} \frac{\mu^{-n}-\lambda^{-n}}{\mu-\lambda} \frac{1}{n} \frac{\partial^{2}}{\partial t_{1} \partial t_{n}} \log \tau_{\mathrm{dKP}}\right),
$$

where $\mu$ and $\lambda$ are free parameters.

Proof. Let $\tau=\tau_{\mathrm{KP}}$ be a tau function of a solution $L$ of the KP hierarchy the quasiclassical limit of which gives the solution $\mathcal{L}$ of the dispersionless KP hierarchy corresponding to $\tau_{\mathrm{dKP}}$ (see Corollary 1.7.6). This means that $\log \tau=\hbar^{-2} \log \tau_{\mathrm{dKP}}+O\left(\hbar^{-1}\right)$.

The differential Fay identity can be written as

$$
\begin{aligned}
& \frac{\tau\left(t-\hbar\left[\mu^{-1}\right]-\hbar\left[\lambda^{-1}\right]\right) \tau(t)}{\tau\left(t-\hbar\left[\mu^{-1}\right]\right) \tau\left(t-\hbar\left[\lambda^{-1}\right]\right)} \\
& =1+\frac{1}{\mu-\lambda}\left(\frac{\partial}{\partial t_{1}} \log \tau\left(t-\hbar\left[\mu^{-1}\right]\right)-\frac{\partial}{\partial t_{1}} \log \tau\left(t-\hbar\left[\lambda^{-1}\right]\right)\right) .
\end{aligned}
$$

The logarithm of the left hand side gives

$$
\sum_{m, n=1}^{\infty} \mu^{-m} \lambda^{-n} p_{n}(-\hbar \tilde{\partial}) p_{m}(-\hbar \tilde{\partial}) \log \tau,
$$


and the logarithm of the right hand side of (B.5), similarly,

$$
\log \left(1+\sum_{n=1}^{\infty} \frac{\mu^{-n}-\lambda^{-n}}{\mu-\lambda} p_{n}(-\hbar \tilde{\partial}) \hbar \frac{\partial}{\partial t_{1}} \log \tau\right)
$$

The leading term of $\hbar$-expansion of (B.6) and (B.7) (the coefficients of $\hbar^{0}$ ) are equal to the left and right hand side of (B.4).

As we shall see below, the differential Fay identity completely characterizes the tau function of the KP hierarchy. Since $\log \tau_{\mathrm{dKP}}$ satisfies the leading term of the differential Fay identity (B.4), we can expect that (B.4) characterizes the tau function of the dispersionless KP hierarchy.

Now we show that the differential Fay identity is equivalent to the whole system of the KP hierarchy. For the notational symplicity, we set $\hbar=1$ below.

Proposition B.3. Let $L$ be a micro-differential operator of the form (1.7.2) and $B_{n}=$ $\left(L^{n}\right)_{\geq 0}$. Define functions $v_{n}(t)$ and polynomials $J_{n, m}[v]$ of $v_{n}$ by

$$
\begin{gathered}
\partial=L+\sum_{m=1}^{\infty} v_{m+1}(t) L^{-m}, \\
J_{n, m}[v]=v_{n+m}+\frac{1}{2} \sum_{\substack{i, i^{\prime}, j, j^{\prime} \geq 1 \\
i+i^{\prime}=m \\
j+j^{\prime}=n}} v_{i+j} v_{i^{\prime}+j^{\prime}}+\frac{1}{3} \sum_{\substack{i, i^{\prime}, i^{\prime \prime}, j, j^{\prime}, j^{\prime \prime} \geq 1 \\
i+i^{\prime}+i^{\prime \prime}=m \\
j+j^{\prime}+j^{\prime \prime}=n}} v_{i+j} v_{i^{\prime}+j^{\prime}} v_{i^{\prime \prime}+j^{\prime \prime}}+\cdots
\end{gathered}
$$

Note that $v_{n}$ are differential polynomials of $u_{m}$ and vice versa.

i) ([13]) The Lax equations

$$
\frac{\partial L}{\partial t_{n}}=\left[B_{n}, L\right], \quad n=1,2, \ldots
$$

and a system of differential equations of $v_{n}$

$$
p_{n}(-\tilde{\partial}) v_{m+1}+\frac{\partial}{\partial t_{1}} J_{n, m}[v]=0, \quad n, m=1,2, \ldots
$$

are equivalent. 
ii) If $\tau(t)$ satisfies the differential Fay identity (B.2) and $v_{n}(t)$ is defined by

$$
v_{n}(t)=\frac{\partial}{\partial t_{1}}\left(p_{n-1}(-\tilde{\partial}) \log \tau\right), n=2,3, \ldots,
$$

then $v_{n}$ satisfy (B.11). Therefore $\tau$ is a tau function for $L$ defined from $v_{n}$ by (B.8).

iii) ([13]) Under the condition (B.10) or (B.11), if a function $\Psi(t ; \lambda)$ of the form (1.7.11) $(\hbar=1)$ satisfies

$$
\begin{gathered}
L \Psi=\lambda \Psi, \\
\frac{\partial \Psi}{\partial t_{n}}=B_{n} \Psi, \quad n=1,2, \ldots,
\end{gathered}
$$

then $\Psi$ satisfies

$$
\begin{gathered}
\frac{\partial}{\partial t_{1}} \Psi=\left(\lambda+\sum_{n=1}^{\infty} v_{n+1}(t) \lambda^{-n}\right) \Psi \\
p_{n}(-\tilde{\partial}) \Psi=v_{n} \Psi, \quad n=2,3, \ldots
\end{gathered}
$$

Conversely, if $\Psi$ of the form (1.7.11) satisfies (B.14), then it also satisfies (B.13).

Proof. Though i) and iii) are stated in [13], there is no proof published. The following is our proof simplified by T. Shiota's comment.

First we prove ii). By definition (B.12), it is easy to see that

$$
v(t ; \lambda) \underset{\text { def }}{=} \sum_{n=1}^{\infty} v_{n+1}(t) \lambda^{-n}=\frac{\partial}{\partial t_{1}}\left(\log \tau\left(t-\left[\lambda^{-1}\right]\right)-\log \tau(t)\right) .
$$

The polynomial $J_{n, m}$ is the coefficient of $\lambda^{-n} \mu^{-m}$ in a generating function

$$
J(\lambda, \mu)=\log \left(1+\frac{v(t ; \lambda)-v(t ; \mu)}{\lambda-\mu}\right) .
$$

Thus, multiplying $\lambda^{-n} \mu^{-m}$ to the left hand side of (B.11) and summing up, we obtain

$$
v\left(t-\left[\lambda^{-1}\right] ; \mu\right)-v(t ; \mu)-\frac{\partial}{\partial t_{1}} J(\lambda, \mu),
$$

which is equal to

$$
\begin{aligned}
\frac{\partial}{\partial t_{1}} \log \frac{\tau\left(t-\left[\lambda^{-1}\right]-\left[\mu^{-1}\right]\right.}{\tau\left(t-\left[\lambda^{-1}\right]\right)}-\frac{\partial}{\partial t_{1}} \log \frac{\tau\left(t-\left[\mu^{-1}\right]\right)}{\tau(t)} & \\
& -\frac{\partial}{\partial t_{1}} \log \left(1+\frac{1}{\lambda-\mu}\left(\frac{\partial_{t_{1}} \tau\left(t-\left[\lambda^{-1}\right]\right)}{\tau\left(t-\left[\lambda^{-1}\right]\right)}-\frac{\partial_{t_{1}} \tau\left(t-\left[\mu^{-1}\right]\right)}{\tau\left(t-\left[\mu^{-1}\right]\right)}\right)\right) .
\end{aligned}
$$


This is identical to 0 due to (B.2). Equation (B.11) is proved.

Next, suppose that (B.11) holds. Note that this system of equations is uniquely solvable if the initial values $\left.v_{n}(t)\right|_{t_{k}=0, k \neq 1}$ are given. In fact, (B.11) can be rewritten into the form

$$
-\frac{1}{n} \frac{\partial}{\partial t_{n}} v_{m+1}(t)=\left(\text { polynomial of } \partial_{t_{1}}, \ldots, \partial_{t_{n-1}}\right) v_{m+1}(t)-\partial_{t_{1}}\left(J_{n, m}[v]\right),
$$

which uniquely determines $\left\{\left.v_{m}(t)\right|_{t_{k}=0, k \geq n+1}\right\}_{m}$ from $\left\{\left.v_{m}(t)\right|_{t_{k}=0, k \geq n}\right\}_{m}$. Let $u_{n}(t)$ be functions defined by (B.8) and $\tilde{L}=\partial+\sum_{n=1}^{\infty} \tilde{u}_{n+1}(t) \partial^{-n}$ be a solution of the KP hierarchy uniquely defined by the initial condition $\left.\tilde{u}_{n}(t)\right|_{t_{k}=0, k \neq 1}=\left.u_{n}(t)\right|_{t_{k}=0, k \neq 1}$. Further we define $\tilde{v}_{n}(t)$ by (B.8) from $\tilde{L}$. By these definitions, $\left.\tilde{v}_{n}(t)\right|_{t_{k}=0, k \neq 1}=\left.v_{n}(t)\right|_{t_{k}=0, k \neq 1}$. By Proposition B.1 and ii) of Proposition B.3 already proved in the first step, $\tilde{v}_{n}(t)$ satisfy the system (B.11). Hence from the unique solvability mentioned above, $\tilde{v}_{n}(t)=v_{n}(t)$, which implies $\tilde{u}_{n}(t)=u_{n}(t)$. Thus (B.10) follows.

Lastly, we prove iii). Suppose that $\Psi$ satisfies (B.13), i.e., $\Psi$ is a Baker-Akhiezer function. Then $\Psi$ is expressed in terms of the tau function as

$$
\Psi=\frac{\tau\left(t-\left[\lambda^{-1}\right]\right)}{\tau(t)} e^{t(\lambda)}
$$

By this formula the generating function of the left hand side of the second equation of (B.14) becomes

$$
\begin{aligned}
& \sum_{n=2}^{\infty} \mu^{-n} p_{n}(-\tilde{\partial}) \Psi(t ; \lambda) \\
= & \frac{\Psi(t ; \lambda)}{\mu}\left((\mu-\lambda) \frac{\tau\left(t-\left[\lambda^{-1}\right]-\left[\mu^{-1}\right]\right) \tau(t)}{\tau\left(t-\left[\lambda^{-1}\right]\right) \tau\left(t-\left[\mu^{-1}\right]\right)}-\mu+\lambda+\frac{\partial_{t_{1}} \tau\left(t-\left[\lambda^{-1}\right]\right)}{\tau\left(t-\left[\lambda^{-1}\right]\right)}-\frac{\partial_{t_{1}} \tau(t)}{\tau(t)}\right),
\end{aligned}
$$

which is equal to $v(t ; \mu) \Psi(t ; \lambda)$ due to the differential Fay identity. Thus the second line of (B.14) is proved. The first equation of (B.14) is an easy consequence of the definition of $v_{n},($ B.8).

Conversely, suppose that a function $\Psi(t ; \lambda)$ satisfying (B.14) is given. The second 
part of (B.14) has a generating function expression

$$
\begin{gathered}
X \Psi=\mu^{-1}\left(\partial_{t_{1}}-v(t ; \mu)\right) \Psi \\
X=1-\exp \left(-\sum_{n=1}^{\infty} \frac{\mu^{-n}}{n} \frac{\partial}{\partial t_{n}}\right) .
\end{gathered}
$$

Since $X$ is a differential operator with costant coefficients, it commutes with itself. Hence,

$$
\sum_{m=1}^{\infty} \frac{1}{m} X^{m}=\sum_{n=1}^{\infty} \frac{1}{n} \frac{\partial}{\partial t_{n}}
$$

Applying both hand sides of this identity to $\Psi$, we obtain from (B.18)

$$
\sum_{n=1}^{\infty} \frac{1}{n} \frac{\partial}{\partial t_{n}} \Psi(t ; \lambda)=\sum_{n=1}^{\infty} \frac{\mu^{-n}}{n} P_{n}(\partial) \Psi(t ; \lambda)
$$

where $P_{n}$ is a differential operator of $n$-th order. Comparing both hand sides of (B.20), we have

$$
\begin{aligned}
P_{n} \Psi(t ; \lambda) & =\frac{\partial}{\partial t_{n}} \Psi(t ; \lambda) \\
& =\left(\lambda^{n}+O\left(\lambda^{-1}\right)\right) \Psi(t ; \lambda) .
\end{aligned}
$$

The first equation of (B.14) implies the first equation of (B.13) because of the definition (B.8). Coupling this equation with (B.21), we have

$$
\left(P_{n}-L^{n}\right) \Psi(t ; \lambda)=O\left(\lambda^{-1}\right) \Psi(t ; \lambda)
$$

This equation means that $P_{n}-L^{n}$ does not contain positive powers of $\partial$. Thus $P_{n}=$ $\left(L^{n}\right)_{\geq 0}=B_{n}$, which proves (B.13) because of (B.21).

\section{REFERENCES}

[1] Takasaki, K., and Takebe, T., SDiff(2) KP hierarchy, in: Proceedings of RIMS Research Project 1991 "Infinite Analysis," Int. J. Mod. Phys. A7, Suppl. 1B (1992), 889-922. 
[2] Takasaki, K., and Takebe, T., SDiff(2) Toda equation - hierarchy, tau function and symmetries, Lett. Math. Phys. 23 (1991), 205-214.

[3] Takasaki, K., and Takebe, T., Quasi-classical limit of KP hierarchy, Wsymmetries and free fermions, Kyoto preprint KUCP-0050/92 (July, 1992), to appear in: Proceedings of Lobachevsky Semester of Euler International Mathematical Institute, 1992, St. Petersburg.

[4] Takasaki, K., and Takebe, T., Quasi-classical limit of Toda hierarchy and Winfinity symmetries, Lett. Math. Phys. 28 (1993), 165-176.

[5] Lebedev, D., and Manin, Yu., Conservation Laws and Lax Representation on Benny's Long Wave Equations, Phys.Lett. 74A (1979), 154-156; Zakharov, V.E., On the Benney's equations, Physica 3D (1981), 193-202.

[6] Kodama, Y., A method for solving the dispersionless KP equation and its exact solutions, Phys. Lett. 129A (1988), 223-226; Kodama, Y., and Gibbons, J., A method for solving the dispersionless KP hierarchy and its exact solutions, II, Phys. Lett. 135A (1989), 167-170; Kodama, Y., Solutions of the dispersionless Toda equation, Phys. Lett. 147A (1990), 477-482.

[7] Krichever, I.M., The dispersionless Lax equations and topological minimal models, Commun. Math. Phys. 143 (1991), 415-426; The $\tau$-Function of the Universal Whitham Hierarchy, Matrix Models and Topological Field Theories, LPTENS92-18, hep-th/9205110, to appear in: Commun. Pure and Appl. Math., XLVII (1994).

[8] Dubrovin, B.A., Integrable systems in topological field theory, Nucl. Phys. B379 (1992), 627-689; Hamiltonian formalism of Whitham-type hierarchies and topological Landau-Ginsburg models, Commun. Math. Phys. 145 (1992), 195207.

[9] Boyer, C., and Finley, J.D., Killing vectors in self-dual, Euclidean Einstein spaces, J. Math. Phys. 23 (1982), 1126-1128; Gegenberg, J.D., and Das, A., 
Stationary Riemannian space-times with self-dual curvature, Gen. Rel. Grav. 16 (1984), 817-829.

[10] Golenisheva-Kutuzova, M.I., and Reiman, A.G., Integrable equations related to Poisson algebras, Zap. Nauch. Semin. LOMI 169 (1988), 44-50 (in Russian); Saveliev, M.V., and Vershik, A.M., Continual analogues of contragredient Lie algebras, Commun. Math. Phys. 126 (1989), 367-378; Kashaev, R.M., Saveliev, M.V., Savelieva, S.A., and Vershik, A.M., On nonlinear equations associated with Lie algebras of diffeomorphism groups of two-dimensional manifolds, Institute for High Energy Physics preprint 90-I (1990).

[11] Bakas, I., The structure of the $W_{\infty}$ algebra, Commun. Math. Phys. 134 (1990), 487-508.; Area preserving diffeomorphisms and higher spin fields in two dimensions, in Supermembranes and Physics in 2+1 Dimensions, Trieste 1989, M. Duff, C. Pope and E. Sezgin eds. (World Scientific, 1990); Park, Q-Han, Extended conformal symmetries in real heavens, Phys. Lett. 236B (1990), 429-432.

[12] Hitchin, N.J., Complex manifolds and Einstein's equations, in Twistor Geometry and Non-linear Systems, H.D. Doebner and T. Weber (eds.), Lecture Notes in Mathematics vol. 970 (Springer-Verlag 1982); Jones, P.E., and Tod, K.P., Minitwistor spaces and Einstein-Weyl spaces, Class. Quantum Grav. 2 (1985), 565-577; Ward, R.S., Einstein-Weyl spaces and $S U(\infty)$ Toda fields, Class. Quantum Grav. 7 (1990), L95-L98; LeBrun, C., Explicit self-dual metrics on $C P_{2} \# \ldots$. . \# $C P_{2}$, J. Diff. Geometry 34 (1991), 223-253.

[13] Sato, M., and Sato, Y., Soliton equations as dynamical systems on infinite dimensional Grassmann manifold, in Nonlinear Partial Differential Equations in Applied Science; Proceedings of the U.S.-Japan Seminar, Tokyo, 1982, Lect. Notes in Num. Anal. 5 (1982), 259-271.

[14] Sato, M., and Noumi, M., Soliton equations and the universal Grassmann manifolds, Sophia University Kokyuroku in Math. 18 (1984), in Japanese. 
[15] Date, E., Kashiwara, M., Jimbo, M., and Miwa, T., Transformation groups for soliton equations, in: Nonlinear Integrable Systems - Classical Theory and Quantum Theory (World Scientific, Singapore, 1983), 39-119.

[16] Takasaki, K., An infinite number of hidden variables in hyper-Kähler metrics, J. Math. Phys. 30 (1989), 1515-1521.

[17] Takasaki, K., Symmetries of hyper-Kähler (or Poisson gauge field) hierarchy, J. Math. Phys. 31 (1990), 1877-1888.

[18] Orlov, A. Yu. and Schulman, E. I., Additional symmetries for integrable equations and conformal algebra representation, Lett. Math. Phys. 12 (1986), 171179; Orlov, A. Yu., Vertex operators, $\bar{\partial}$-problems, symmetries, variational indentities and Hamiltonian formalism for $2+1$ integrable systems, in: Plasma Theory and Nonlinear and Turbulent Processes in Physics (World Scientific, Singapore, 1988); Grinevich, P. G., and Orlov, A. Yu., Virasoro action on Riemann surfaces, Grassmannians, $\operatorname{det} \bar{\partial}_{j}$ and Segal Wilson $\tau$ function, in: Problems of Modern Quantum Field Theory (Springer-Verlag, 1989).

[19] Sato, M., Kawai, T. and Kashiwara, M., Microfunctions and pseudo-differential equations, Lecture Notes in Math. 287 (1973), 265-529; Kashiwara M., Systems of Microdifferential Equations, (1983), Birkhäuser; Schapira P., Microdifferential Systems in the Complex Domain, (1985), Springer.

[20] Mulase, M., Complete integrability of the Kadomtsev-Petviashvili equation, Advances in Math. 54 (1984), 57-66; Solvability of the super KP equation and a generalization of the Birkhoff decomposition, Invent. Math. 92 (1988), 1-46.

[21] Ueno, K., and Takasaki, K., Toda lattice hierarchy, in Group Representations and Systems of Differential Equations, K. Okamoto ed., Advanced Studies in Pure Math. 4 (North-Holland/Kinokuniya 1984), 1-95.

[22] Eguchi, T., and Kanno, H., Toda lattice hierarchy and the topological description of the $c=1$ string theory, University of Tokyo preprint UT-674, 
hep-th/9404056 (April, 1994); Hanay, A., Oz, Y., and Plesser, M.R., Topological Landau-Ginzburg formulation and integrable structure of $2 \mathrm{~d}$ string theory, IASSNS-HEP 94/1, hep-th/9401030 (January, 1994); Takasaki, K., Dispersionless Toda hierarchy and two-dimensional string theory, Kyoto preprint KUCP-0067/94, hep-th/9403190 (March, 1994); Bonora L. and Xiong, C. S., Two-matrix model and $c=1$ string theory, SISSA-ISAS 54/94/EP, BONN-HE06/94, hep-th/9405004.

[23] Krichever, I.M., Method of averaging for two-dimensional "integrable" equations, Funkts. Anal. Pril. 22-3 (1988), 37-52 (in Russian); Funct. Anal. Appl. 22 (1988), 200-213 (Engl. transl.).

[24] Penrose, R., Nonlinear gravitons and curved twistor theory, Gen. Rel. Grav. 7 (1976), 31-52.

[25] Hitchin, N.J., Karlhede, A., Lindström, U., and Roček, M., Hyperkähler metrics and supersymmetry, Commun. Math. Phys. 108 (1987), 535-589.

[26] Boyer, C.P., and Plebanski, J.F., An infinite hierarchy of conservation laws and nonlinear superposition principles for self-dual Einstein spaces, J. Math. Phys. 26 (1985), 229-234.

[27] Takasaki, K., Differential algebras and $\mathcal{D}$-modules in super Toda lattice hierarchy, Lett. Math. Phys. 19 (1990), 229-236.

[28] Kac, V.G., Infinite dimensional Lie algebras, 3rd ed., (1990), Cambridge Univ. Press.

[29] Witten, E., Ground ring of two dimensional string theory, Nucl. Phys. B373 (1992), 187-213.

[30] Dijkgraaf, R., Verlinde, H., and Verlinde, E., Topological strings in $d<1$, Nucl. Phys. B352 (1991), 59-86; Loop equations and Virasoro constraints in non-perturbative 2d quantum gravity, Nucl. Phys. B348 (1991), 435-456.

[31] Dubrovin, B.A., and Novikov, S.P., Hamiltonian formalism of one-dimensional 
systems of hydrodynamic type and Bogolyubov-Whitham averaging method, Dokl. Akad. Nauk SSSR 270 (1983), 781-785 (in Russian); Soviet Math. Dokl. 27 (1983), 665-669 (Engl. transl.).

[32] Tsarev, S.P., On Poisson brackets and one dimensional Hamiltonian systems of hydrodynamic type, Dokl. Akad. Nauk SSSR 282 (1985), 534-537 (in Russian); Soviet Math. Dokl. 31 (1985), 488-491 (Engl. transl.).

[33] Losev, A.S., and Polyubin, I., On connection between topological LandauGinzburg gravity and integrable systems, hep-th/9305079 (May, 1993); Eguchi, T., Kanno, H., Yamada, Y., and Yang, S.-K., Topological strings, flat coordiantes and gravitational descendents, Phys. Lett. B305 (1993), 235-241; Eguchi, T., Yamada, Y., and Yang, S.-K., Topological field theories and the period integrals, Mod. Phys. Lett. A8 (1993), 1627-1638.

[34] Kontsevich, M., Intersection theory on the moduli space of curves and the matrix Airy function, Commun. Math. Phys. 147 (1992), 1-23.

[35] Kharchev, S., Marshakov, A., Mironov, A., Morozov, A., and Zabrodin, A., Towards unified thoery of 2-d gravity, Nucl. Phys. B380 (1992), 181-240; Itzykson, C., and Zuber, J. B., Addendum to: Combinatorics of the modular group 2., the Kontsevich integrals, Intern. J. Mod. Phys. A7 (1992), 5661-5705; Kharchev, S., Marshakov, A., Mironov, A., and Morozov, A., Landau-Ginzburg topological theories in the framework of GKM and equivalent hierarchies, Mod. Phys. Lett. A8 (1993) 1047-1062; Generalized Kontsevich model versus Toda hierarchy and discrete matrix models, Nucl. Phys. B397 (1993), 339-378.

[36] Fukuma, M., Kawai, H., and Nakayama, R., Continuum Schwinger-Dyson equations and universal structures in two-dimensional quantum gravity, Int. J. Mod. Phys. A6 (1991), 1385-1406; Infinite dimensional Grassmannian structure of two dimensional string theory, Commun. Math. Phys. 143 (1991), 371-403.

[37] Adler, M., and van Moerbeke, P., A matrix integral solution to two-dimensional 
$W_{p}$-gravity, Commun. Math. Phys. 147 (1992), 25-56.

[38] Nakatsu, T., Kato, A., Noumi, M., and Takebe, T., Topological strings, matrix integrals, and singularity theory, Phys. Lett. B322 (1994), 192-197; Kato, A., Nakatsu, T., Noumi, M., and Takebe, T. in preparation.

[39] Takebe, T., Toda lattice hierarchy and conservation laws, Commun. Math. Phys. 129 (1990), 281-318.

[40] Jimbo, M., and Miwa, T., Solitons and infinite dimensional Lie algebras, Publ. RIMS, Kyoto Univ. 19 (1983), 943-1001.

[41] Takebe, T., Representation theoretical meaning of the initial value problem for the Toda lattice hierarchy: I, Lett. Math. Phys. 21 (1991), 77-84; ditto II, Publ. RIMS, Kyoto Univ., 27 (1991), 491-503.

[42] Takasaki, K., Initial value problem for the Toda lattice hierarchy, in Group Representations and Systems of Differential Equations, K. Okamoto ed., Advanced Studies in Pure Math. 4 (North-Holland/Kinokuniya 1984), 139-163.

[43] Kodama, Y., and Gibbons, J., Integrability of the dispersionless KP hierarchy in: Proceedings of Workshop "Nonlinear and Turbulent Processes in Physics", Kiev 1989 (World Scientific, 1990), 160-180. 